\title{
7. Queere Diskurse: Zur Konstitution eines kollektiven Wir
}

Für die Bewegungsöffentlichkeiten der fünf Projekte sind, je nach Arbeitsschwerpunkt, unterschiedliche Themen und Medien zentral: LesMigraS verwendet vorwiegend Broschüren und Pressemitteilungen/Stellungnahmen, um Gewalt und den möglichen Umgang damit, sowie Rassismus und Transphobie in der Community zu thematisieren. Bei TrIQ werden ebenfalls vorwiegend Broschüren und Pressemitteilungen/Stellungnahmen genutzt, um auf Pathologisierung und Normierung in medizinischen und medialen Diskursen aufmerksam zu machen. Die Türkis Rosa Lila Villa (im Folgenden kurz: Villa) verweist mit Hilfe von Bannern auf der Hausfassade, einem Blog und Artikeln in den »Lambda-Nachrichten« auf die Situation von queeren Geflüchteten. In den Pressemitteilungen/Stellungnahmen von Lambda BB sind die Forderungen nach mehr Räumen und Ressourcen für das eigene Projekt und Mehrfachdiskriminierung zentral, und die Verbandszeitschrift »Out! « dreht sich um das Coming-out. Das »Milchbüechli«, die Zeitschrift der Milchjugend, ist um die Pride und den damit verbundenen Stolz und die Widerständigkeit angeordnet. Neben diesen für die einzelnen Projekte spezifischen Öffentlichkeiten gibt es aber auch Themen, die projektübergreifend vorkommen und die entsprechend für den queer-aktivistischen Diskurs im deutschsprachigen Raum zentral sind. Auf Grundlage der strukturanalytischen Aufarbeitung des gesamten Materials sind drei Diskursstränge ${ }^{1}$ für die Feinanalyse ausgewählt worden, die in allen Projekten verhandelt werden:

a) die Pride,

b) Mehrfachdiskriminierung,

c) das Coming-out.

Wesentliche Auswahlkriterien waren erstens die Repräsentativität der Themen und zweitens die Vergleichbarkeit und Relevanz für die Fragestellung. 
Ein zentrales Ereignis der analysierten queer-aktivistischen Diskurse stellen die jährlich stattfindenden Pride-Paraden dar. Die queeren Projekte konstituieren sich über die Verhandlung der Frage, ob und auf welche Art und Weise sie als ein kollektives Wir auf der Pride in Erscheinung treten. Ein zweiter zentraler Diskursstrang, der in allen Projekten thematisiert wird, sind die Debatten zu Mehrfachdiskriminierung, insbesondere mit Bezug auf Rassismus. In diesen Diskursen geht es wesentlich darum, auf welche Art und Weise sich die Projekte in Bezug auf Rassismus als solidarische Gemeinschaften herstellen. Nachdem alle Projekte auch Unterstützungs-, Beratungsund Austauschorte sind, ist der letzte ausgewählte Diskursstrang - das Coming-out ein Thema, um das viele der jeweiligen Gemeinschaften wesentlich angeordnet sind. Hier konstituiert sich das kollektive Wir der Projekte in Abhängigkeit von der Frage, wie das individuelle Wohlbefinden der einzelnen Mitglieder hergestellt werden kann. Im Folgenden soll nun entlang dieser drei Themen aufgezeigt werden, auf welche Art und Weise in den queer-aktivistischen Diskursen ein kollektives Wir hergestellt wird. Zentral ist dabei - vor dem Hintergrund einer queeren Kritik an eindeutigen Identitätspolitiken - die Frage, welche Rolle Identitäten dabei spielen. Für jedes der drei zentralen Themen - Pride, Mehrfachdiskriminierung und Coming-out - wird die Feinanalyse von zwei ausgewählten, repräsentativen Diskursfragmenten der fünf im Zentrum der vorliegenden Untersuchung stehenden Projekte ausführlich dargestellt. Die Erkenntnisse aus den Feinanalysen der repräsentativen Diskursfragmente der anderen drei Projekte werden im Anschluss jeweils verdichtet beschrieben.

\subsection{Pride-Paraden: Umkämpfte Orte queerer Politiken}

Das durch alle Projekte hindurch am häufigsten verhandelte Thema sind die Pride-, Regenbogen- beziehungsweise CSD-Veranstaltungen. ${ }^{2}$ Im Materialkorpus ist der CSD oder die Pride- beziehungsweise die Regenbogenparade in zwanzig Diskursfragmenten auf die eine oder andere Weise Thema. Bei den Diskursfragmenten handelt es sich um Stellungnahmen in Form von Redebeiträgen oder schriftlichen Statements, die auf der Homepage der jeweiligen Projekte downloadbar sind, sowie um Artikel, die im Falle der Milchjugend und des Jugendnetzwerks Lambda in den projektinternen Zeitschriften, im Falle von TrIQ auf der Homepage des »Gunda-Werner-Instituts « und im Fall der Villa in den »Lambda-Nachrichten ${ }^{3}$ veröffentlicht wurden. ${ }^{4}$ Alle fünf Projekte nehmen

Die Milchjugend aus der Schweiz spricht von Pride-Paraden, die Villa von Regenbogenparaden und die Berliner Projekte sprechen meistens von CSD-Paraden. Alle übernehmen die in ihrer Stadt gängigen Bezeichnungen. Im Folgenden werden diese Begriffe abwechselnd verwendet, wenn auf alle drei Länder Bezug genommen wird. Ansonsten wird der Begriff verwendet, der von den jeweiligen Projekten verwendet wird.

3 Wie in Kapitel sechs bereits beschrieben wurde, veröffentlichte die Villa in den Jahren 2014 bis 2016 Artikel in den »Lambda-Nachrichten«, die von der »Hosi« in Wien herausgegeben werden.

$4 \quad$ Von den neun Artikeln zur Pride sind zwei Artikel vom Jugendnetzwerk Lambda, jeweils ein Artikel von TrlQ und der Türkis Rosa Lila Villa und fünf Artikel von der Milchjugend verfasst worden. Von den insgesamt elf Stellungnahmen zur Pride sind sechs von LesMigraS, zwei vom Jugendnetzwerk Lambda BB und drei von TrIQ. 
meistens an den CSD-Veranstaltungen teil. Entsprechend positionieren sie sich in ihren Stellungnahmen und Artikeln zu den CSD-Paraden, halten Reden auf den Veranstaltungen, berichten von ihnen oder diskutieren sie in ihren Artikeln. In den zum CSD entstandenen Diskursfragmenten sind drei Aspekte zentral, die alle um die Frage, ob und wie die jeweiligen Projekte auf den Paraden in Erscheinung treten, angeordnet sind und wechselseitig aufeinander Bezug nehmen: der bewegungsgeschichtliche Kontext, die Kommerzialisierung der Pride und rassistische sowie transphobe Ausschlüsse.

Projektübergreifend ist den Verhandlungen um die Pride gemeinsam, dass die Pride als ein ([un]möglicher) Ort des Politischen diskutiert wird. Die Projekte konstituieren sich alle insofern als ein kollektives Wir, als sie im Sprechen über den CSD verhandeln, ob und wie sie an den Paraden teilhaben. Dabei geben sie unterschiedliche Antworten auf die Frage, was das Politische ist, und eng damit verbunden auch, inwiefern die Pride (k)ein Ort des Politischen für sie ist. Dahinter steckt der alle Texte durchziehende Wunsch: Die Pride soll ein Ort des Politischen sein. Nachfolgend wird nun zunächst anhand von Ergebnissen der Feinanalyse repräsentativer Diskursfragmente aufgezeigt, wie die jeweiligen Projekte ihr Verhältnis zur Pride ausloten. Das Aushandeln des spezifischen Verhältnisses der Projekte zum CSD wird als ein Ort verstanden, an dem sich das jeweilige kollektive Wir konstituiert. Zwei Diskursfragmente - eins von der Milchjugend, "Denk mal nach: Bringt's die Pride?! ${ }^{5}$, und eins von LesMigraS, »Mehrdimensionales Empowerment statt eindimensionaler Feiertag ${ }^{6}$ - sind für die ausführliche Ergebnisdarstellung ausgewählt worden. Dabei handelt es sich im Fall der Milchjugend um einen Artikel im »Milchbüechli«, in dem diskutiert wird, ob die Milchjugend an der Pride teilnehmen sollte, und im Fall von LesMigraS um eine Stellungnahme, in der die Einrichtung erklärt, warum sie dieses Jahr nicht am CSD teilnimmt. Beide Diskursfragmente wurden für die ausführliche Ergebnisdarstellung ausgewählt, weil das Thema in diesen beiden Projekten besonders häufig verhandelt wird, beide die Pride auf eine unterschiedliche Art diskutieren und es sich jeweils um die beiden Textsorten handelt, mittels derer die Pride diskutiert wird. ${ }^{7}$ Darüber hinaus wird von den anderen Projekten jeweils ein Diskursfragment verdichtet dargestellt. ${ }^{8}$

6 LesMigraS: Pressemitteilung anlässlich des Christopher Street Days. Mehrdimensionales Empowerment statt eindimensionaler Feiertag 2014, https://lesmigras.de/tl_files/lesmigras/pressemi tteilungen/PM_2014_CSD.pdf vom 26.09.2020.

7 Dabei ist zu beachten, dass diese unterschiedliche Positionierung auch (allerdings nicht nur) darauf zurückzuführen ist, dass die Prides in den jeweiligen Städten unterschiedlich organisiert sind beziehungsweise dort verschiedene Debatten aktuell sind.

8 Beim Jugendnetzwerk Lambda BB und bei TrIQ handelt es sich jeweils um einen Redebeitrag, der am CSD gehalten wurde. Es wurde aber auch ein Artikel aus der»Out!«, der Verbandszeitschrift des Jugendnetzwerks Lambda BB, zum CSD sowie ein Artikel der Villa in den »Lambda-Nachrichten«, in denen die Regenbogenparade 2014 Thema ist, ausgewählt. Da in der »Out! « und den Pressemitteilungen des Jugendnetzwerks Lambda BB unterschiedliche Positionierungen stattfinden, sind beide Textfragmente in die Ergebnisdarstellung eingeflossen. 


\subsubsection{Die Pride als Ort für die Milchjugend?}

Für die Milchjugend ist die Pride eine von neun Welten. Gemeinsam nehmen die Jugendlichen regelmäßig an jeder Pride-Veranstaltung in der Schweiz teil. Vor allem auf der Zürich-Pride ist die Milchjugend auf vielfältige Weise präsent: Die Milchjugend hat einen eigenen Wagen, einen eigenen Stand auf dem Festivalgelände, halten Reden auf der Politbühne oder vor der Parade und veranstalten im Anschluss an die Parade eine eigene Party. Der finanzielle Aufwand für die Jugendpride liegt laut Rechnungen und Budgetplanungen aus dem Jahr 2018/19 bei ca. 6500 Schweizer Franken. ${ }^{9}$ Nachdem die Milchjugend regelmäßig an der Pride teilnimmt, sind die Widersprüche, aber auch die Erfahrungen, die auf der Pride gemacht werden, immer wieder Anlass für Artikel, die im »Milchbüechli« veröffentlicht werden. Insgesamt hat die Milchjugend in den 18 Ausgaben zwischen 2012 und 2016 fünf Artikel zur Pride verfasst. Hinzu kommen Artikel, in denen das Motto der Pride - meistens »die Ehe für alle« - diskutiert wird. Neben dem 2013 erschienenen, für die Feinanalyse ausgewählten Diskursfragment zur Pride wurde in der Juni-Ausgabe 2014 die Pride auf einer Doppelseite unter dem Titel »Zürich-Pride Festival« gleichzeitig als Veranstaltung beworben und deren politische Relevanz diskutiert. Im März 2015 veröffentlicht die Milchjugend einen Artikel zu den historischen Anfängen der Pride - den »Stonewall Inn«-Protesten - (»Where the Pride began«). In einem weiteren Beitrag Ende des Jahres 2015 berichten die Jugendlichen von ihren Pride-Erlebnissen (»Milchbüechli - Pride Sommer«). Ein Jahr später wird die Tatsache, dass die Milchjugend am gleichen Tag, an dem das Massaker in Orlando stattgefunden hat, im Anschluss an die Pride eine Party im »Heaven « gefeiert hat, Anlass zur Reflexion (»Regenbogen-Orte«).

Der für die Analyse ausgewählte Artikel ist ein im Juni 2013 erschienener Kommentar mit dem Titel: »Denk mal nach: Bringt's die Pride?! «. ${ }^{10}$ Der Kommentar wurde in der vierten Ausgabe des »Milchbüechli« veröffentlicht. Es ist die Juni-Ausgabe - also Pride-Zeit. Der Artikel zur Pride steht nicht nur in einem Bezug zu der Pride als aktuell anstehendem Ereignis, sondern ist auch in zweifacher Hinsicht mit dem Themenschwerpunkt der vierten Ausgabe des »Milchbüechli« - »Triff uns Live!« - verknüpft. Die Pride - als konkrete Veranstaltung - ist eine der Möglichkeiten, die Milchjugend »Live« zu treffen. Hier hat der Begriff "Live« die im deutschsprachigen Raum gängige Bedeutung von »direkt«, »unmittelbar«, »leibhaftig«. Das Thema der Ausgabe, »Triff uns Live!«, kann aber auch anders verstanden werden. So verweist das Editorial der vierten Ausgabe implizit auf eine mögliche andere, leicht verschobene Bedeutung des Begriffs »Live«: »IM MILCHBÜECHLI ERLEBST DU DIE VIELFALT DER FALSCHSEXUELLEN JUGEND: WIR SCHREIBEN FÜR DICH [...] STOLZ UND SELBSTBEWUSST AUS UNSEREM LEBEN.«Das »Milchbüechli« als Heft - so legt es das Editorial nahe ermöglicht es, »Live« aus dem Leben der Milchjugend zu erfahren. Die Bedeutung des Begriffs »Live« als »direkt« »unmittelbar« oder auch »leibhaftig« wird in dieser Lesart in mehrfacher Hinsicht verschoben. Die zeitliche Dimension der Bezeichnung »Live«

Das betrifft die Rechnungen und Prognosen für das Jahr 2018/19. Vermutlich werden damit hauptsächlich Stand- und Wagengebühren gezahlt. Milchjugend: Rechnung 2018, 2019. 
wird insofern erweitert, als eine Zeitschrift, die viermal im Jahr erscheint, nicht derart »aktuell« sein kann wie beispielsweise ein »Livestream«. Die Leser*innen erfahren auch nicht »unmittelbar« aus dem Leben der Milchjugend, sondern vermittelt, über geschriebene Texte im Medium Zeitschrift. Entsprechend ist auch die Materialität eine andere. Es handelt sich nicht um die »leibhaftige « Begegnung von »Körpern auf der Straße ", ${ }^{11}$ wie es auf der Pride der Fall wäre. Die Leser*innen haben eine Zeitschrift - die Medium der geschriebenen Texte ist - in der Hand. Der geschriebene Text ist aber - und hier kann ein weiterer Bezug zwischen dem Thema des Artikels und dem Thema der Ausgabe hergestellt werden - nicht nur ein Ort, an dem »Live« aus dem Leben der Milchjugend berichtet wird, sondern auch ein Ort, an dem die Milchjugend »stolz« und »selbstbewusst « aus ihrem Leben schreibt. Der Stolz und das Selbstbewusstsein, die im »Milchbüechli« "Live« hergestellt werden, gehen bewegungsgeschichtlich auf die Pride als ein Ereignis, das eine Politik des Stolzes repräsentiert, zurück. »Triff uns Live!« als Möglichkeit, die Milchjugend auf der Pride zu treffen, und als Möglichkeit, die im »Milchbüechli« hergestellte stolze, selbstbewusste Gemeinschaft der Milchjugend kennenzulernen, sind zwei Aspekte, in denen der Titel der Ausgabe und das Thema des Artikels aufeinander Bezug nehmen.

In Ausgabe vier wird des Weiteren über Regenbogenfamilien berichtet, der aktivistische Verein »Homo Sapiens« vorgestellt, von den Coming-out-Erfahrungen einer Trans*-Person erzählt, ein Kommentar über die normativen Vorstellungen in der Community abgedruckt sowie über einen Aufenthalt in Brighton, das "Coming Inn « - einen Jugendtreff in Bern - und eine sogenannte »Bärenparty« berichtet. Die Autor*in des Artikels »Denk mal nach: Bringt's die Pride?!« hat über einen längeren Zeitraum den Inhalt der Zeitschrift mitgeprägt und eine wichtige Rolle in der Milchjugend ge-

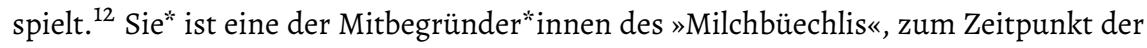
Veröffentlichung 25 Jahre alt und studiert - wie am Ende des Artikels zu lesen ist Gender Studies in Basel. Es ist ihr* vierter Artikel im Milchbüechli. Später arbeitet sie* als Textverantwortliche und dauerhaftes Redaktionsmitglied weiterhin regelmäßig für das Heft.

Bei einer ersten Betrachtung der »Text-Oberfläche ${ }^{13}$ fällt auf, dass der auf einer Doppelseite gedruckte Artikel in drei vertikale Textspalten aufgeteilt ist. Die Spalten sind durch bunte Balken in den Farben Blau, Rosa und Gelb auf der linken Seite des Textes voneinander abgetrennt. Zwischen der ersten und der zweiten Textspalte ist ein Bild von einer Regenbogenflagge, die von Seifenblasen umgeben ist, abgedruckt. Oben rechts befindet sich ein schwarzer Kreis mit blau-rosa Zacken, in dem in weißer Schrift "Pride 2013" geschrieben steht. Die Graphik und das Bild rufen die Leser*innen als Pride-Teilnehmer*innen an und bejahen auf den ersten Blick bereits die Frage aus der

11 J. Butler: Anmerkungen zu einer performativen Theorie der Versammlung, S. 77.

12 Es wird bewusst auf die Nennung der Namen der Autor*innen verzichtet, weil es um die Projekte und nicht um einzelne Personen geht. Der Hintergrund der Autor*innen wird allerdings erwähnt, weil dieser ein zentraler Aspekt der Beschreibung des institutionellen Rahmens eines Diskursfragmentes im Sinne Jägers ist. 
Überschrift, ob’s die »Pride bringt«. Die Überschrift wird von einem schwarzen Balken gerahmt. Oben steht zunächst »Denkt mal nach:« und darunter in fetten Buchstaben »Bringt's die Pride?!«. Unter der Überschrift wird ein kurzer Überblick über den Inhalt des Artikels gegeben: »Ist die Pride ein Umzug der Falschsexuellen? Geht es nicht einfach darum, dass ein paar Firmen neue Kund_innen gewinnen können? Laura geht der Frage nach, ob die >Pride` in Zürich noch was bringt." Bereits die Überschrift ruft die Leser*innen in dreifacher Weise an: als denkende Subjekte - durch die Aufforderung "Denk mal nach «-, als potentielle Pride-Teilnehmer*innen oder zumindest an der Pride Interessierte, aber auch als Jugendliche - wenn im Jugendjargon gefragt wird: »Bringt's die Pride?!«. Der in der Aufforderung »Denk mal nach« verwendete Numerus - die Singularform - ruft zunächst einmal nur die einzelne Leser*in als »denkendes« Subjekt an. Diese singuläre Anrufung wird im folgenden Satz dann aber sogleich gekoppelt an eine kollektive Anrufung, indem die Art und Weise, in der über den Gegenstand - die Pride - nachgedacht werden soll, festgelegt wird. Die Pride »bringt's«dann, wenn sie noch ein »Umzug für die Falschsexuellen« ist. Über die Frage »Bringt's die Pride?« nachzudenken, heißt also nicht, darüber nachzudenken, ob sie der einzelnen Leser*in etwas »bringt«, sondern ob sie den Leser*innen als Teil der »falschsexuellen« Gemeinschaft etwas »bringt«. Hier wird die einzelne Leser"in also zugleich auch als Teil der »Falschsexuellen« angerufen. Ein spezifisches Moment des kollektiven Wir der »Falschsexuellen« wird hier an die Frage nach dem Potential (»Bringen«) der Pride geknüpft. Wann die Pride den »Falschsexuellen« etwas »bringt« und wann nicht und was sie »bringen « soll, wird in den folgenden Abschnitten diskutiert. Dabei wird das spezifische Verhältnis der Milchjugend zur Pride ausgelotet. Der Artikel ist in sechs Abschnitte unterteilt, die formal durch Absätze markiert sind.

Im ersten Abschnitt wird von persönlichen Gefühlen der Euphorie berichtet, die die Autorin bei ihrer ersten Pride-Teilnahme selbst erlebt hat. Dem Gefühl, »Teil einer Bewegung« zu sein, folgten auf den nächsten Pride-Besuchen Momente der Ernüchterung: »Muskeltypen«, die »tanzen« und »feiern« anstatt zu politisieren, »Werbeflyer « statt »aktivistische[r] Flyer« und »Anmachsprüche zum Gratis-Kondom«.

In diesem einleitenden Abschnitt wird die Pride als Ort affektiver Anrufungen beschrieben: »Das erste Mal war euphorisierend [...]. Ich bin Teil einer Bewegung«. Die Autor*in beschreibt die affizierende Wirkung, die eine Massenbewegung haben kann, und positioniert sich hier als Person, die sich selbst diesen Affekten unterworfen hat. Dann wird - noch immer am Beispiel der Pride-Erfahrungen der Autor*in illustriert - ein weiterer, mit der Euphorie in einem Spannungsverhältnis stehender, durch die Pride ausgelöster Affekt beschrieben. Mitunter überspitzt und mit Hilfe von Wortspielen wird von einem zunächst noch nicht »benennbaren« Unbehagen berichtet: »Eigentlich sind es ja immer dieselben, geschniegelten nackten Muskeltypen, die da tanzen. $\mathrm{Ob}$ die $\mathrm{zu}$ Bewegungen fähig sind, die nichts mit ihren Hüften zu tun haben, ist eher fraglich.« Die über positive Affekte der Euphorie wirkende Anrufung wird durch Affekte des Unbehagens gebrochen - ausgelöst durch Werbung, sexistische Sprüche und Ignoranz in Bezug auf Politisches. Dabei verweisen die als Gegensatzpaare aufgeführten Beispiele bereits auf den Maßstab, der darüber entscheidet, ob die Pride noch »ein Umzug der Falschsexuellen« ist: »Statt mit politischen Botschaften, füllen sich meine 
Taschen mit Werbung [...]; Reporter*innen jagen >Paradiesvögek [...] und rennen dabei an Transparenten mit politischen Botschaften vorbei«. Durch die Herstellung von Gegensätzen - politische Botschaften/Werbung, Paradiesvögel/politische Transparente - wird bereits auf den das Unbehagen auslösenden Mangel - jenen des Politischen verwiesen. Die durch die Erlebnisse hervorgerufenen widersprüchlichen Affekte rufen zum Nachdenken auf. Dabei überlappen sich der Text und die durch den Text vermittelte »Wirklichkeit«. Der Text unterwirft sich der eigenen Anrufung: »Denk mal nach«. Er ist selbst ein Ort, an dem über die Pride nachgedacht wird, ruft aber gleichzeitig auch die Leser*innen als »denkende Subjekte« an: »Denk mal nach«.

Das Spezifische der »falschsexuellen « Gemeinschaft - die unter anderem durch das Ausloten des eigenen Verhältnisses zur Pride hergestellt wird - beginnt mit einem »Unbehagen«. Ähnlich beschreibt auch Sara Ahmed, vier Jahre später, den Weg zum Feminismus - »Becoming Feminist«:

»Feminism often begins with intensity: Over time and with experience, you sense an injustice. You might not have used that word for it. You might not have the words for it; you might not be able to put your fingers on it. Feminism can begin with a body, a body in touch with a world, a body that is not at ease in world; a body that fidges and moves around. Things don't seem right. «" 14

Auch das Unbehagen, das als ein erstes, die »falschsexuelle« Gemeinschaft bestimmendes Verhältnis zur Pride beschrieben wird, ist zunächst nicht benennbar. Es entsteht mit der Zeit und der Erfahrung und umfasst das Gefühl, dass etwas nicht richtig ist. Mit Sara Ahmed gesprochen: »Becoming falschsexuell«, insofern, als das »falschsexuelle» Wir sich unter anderem in einem spezifischen Verhältnis zur Pride konstituiert, »begins with intensity«. Gleichzeitig verweisen die Gegensatzpaare (politische Botschaften/Werbung, Paradiesvögel/politische Transparente) bereits auf eine Kritik an der aktuellen Pride im Namen einer »eigentlichen« Pride, die politisch sein soll.

Die innere Spannung - das Unbehagen - wird im zweiten Abschnitt dann in Worte gefasst. »Viele« würden kritisieren, dass die Pride sich von ihrem Ursprung, den »Stonewall Riots«, entfernt habe und stattdessen nur noch eine kommerzielle Party sei. Dabei sei unklar, ob das Feiern noch politisch ist oder die Kommerzialisierung von Vielfalt im Vordergrund stehe. Ökonom*innen hätten schon früh erkannt, dass sich Diversität gut verkaufen lasse.

Der zweite Abschnitt beginnt mit einer Positionierung der Milchjugend als Teil einer größeren Community: »Dieses Unbehagen teilen viele«. Der gemeinsame Nenner ist zunächst das »Unbehagen« als gemeinsam erlebte Emotion. Dabei bleibt unbestimmt und unbenannt, wen die Bezeichnung »viele« umfasst. »Viele« haben aber das »Unbehagen« bereits benannt. Teil der »falschsexuellen« Gemeinschaft zu sein, heißt auch, Teil einer Community zu sein, die das »Unbehagen« bereits in Worte gefasst hat. Noch wird aber keine Position zur Kritik »vieler« bezogen. Vielmehr wird eine Distanz zu den Versuchen, das gemeinsam geteilte Unbehagen zu benennen, aufrechterhalten. Das zeigt sich in der Verwendung der Konjunktivform, in der die Kritik »vieler« vorgetragen wird: 
»Prides [...] hätten mit ihrem Ursprung [...] kaum noch was zu tun. [...] Stattdessen seien sie zu einer Massenveranstaltung geworden«. Im Verlauf des Abschnitts nimmt der Text diese Distanz ein wenig zurück, indem auf einen Blick in die eigene Tasche verwiesen wird, der die Kommerzialisierung »zu bestätigen [...] scheint«. Der Begriff "scheint « fordert aber auch dazu auf, weiterhin Zurückhaltung zu wahren, könnte doch selbst der Blick in die Tasche noch illusorisch sein. Im Gegensatz zu den Distanzierungen gegenüber den Kritiken »vieler« wird dann gegen Ende dieses Abschnitts zumindest ein Phänomen als "wahres« anerkannt: Der Absatzmarkt profitiert von Diversität. Das »Wahre« dieser Aussage wird durch den Einsatz machtvoller Sprecher*innen festgeschrieben, indem Bezug genommen wird auf »einige kluge Ökonom*innen«: »Denn Diversität, so die Entdeckung einiger kluger Ökonom_innen [...], muss ja gar nicht schlecht sein -, im Gegenteil! Unterschiedliche Menschen bedeutet unterschiedliche Bedürfnisse, bedeutet eine ideale Gelegenheit, Spezialangebote an die Menschen zu bringen.« Die Ökonom*innen, über deren wissenschaftliche Autorität die Aussage, dass Diversität kommodifizierbar sei, als Wahrheit hergestellt wird, bleiben allerdings anonym. Es wird entweder ein Vertrauen der Leser*innen oder die Bekanntheit dieser Ökonom*innen vorausgesetzt. Die Aussage »Diversität ist kommodifizierbar« wird zusätzlich auch auf einer emotional-affektiven Ebene als »wahr«bejaht: "Natürlich schlägt unser Herz höher [...], wenn wir eine regenbogenfarbene Wodkaflasche [...] entdecken.« Damit wird die Möglichkeit eröffnet, dass sowohl jene Jugendlichen, die einen Bezug zur wissenschaftlichen Theorie haben, die Aussage als wahr annehmen können als auch jene, »deren Herzen höherschlagen«, wenn sie »Regenbogenprodukte« sehen. Bis hierhin ist die »falschsexuelle« Gemeinschaft eine, die durch widersprüchliche, affektive Anrufungen der Pride ein »Unbehagen« empfindet. Gleichzeitig ist die Milchjugend Teil einer Community, mit der sie dieses »Unbehagen« teilt, zu deren Kritiken an der Pride aber zunächst Distanz gewahrt wird. Bejaht wird vorerst nur, was die Wissenschaft, aber auch das Herz bestätigt, nämlich dass Diversität vom Markt vereinnahmt werden kann.

Im dritten Abschnitt wird gefragt, ob die Kommodifizierung von Diversität nicht eine Win-win-Situation sei, um im Anschluss auf diejenigen zu verweisen, die in diesem Fall nichts zu gewinnen haben. Doch - so argumentiert der Text weiter - wo sei der Markt denn nicht als »Trittbrettfahrer mit dabei!? Das halte doch niemanden davon $a b$, trotzdem politische Transparente zu tragen.

$\mathrm{Zu}$ Beginn dieses Abschnitts wird zunächst das Für und Wider der zuvor bejahten Aussage, dass Diversität kommodifizierbar sei, beleuchtet. In Form von rhetorischen Fragen wird bewusst naiv gefragt, wo denn »das Problem« sei: »Das ist doch eine Winwin-Situation. «Im Anschluss wird - wieder in Form von rhetorischen Fragen - aufgezeigt, wen diese Win-win-Situation ausschließt: »Was passiert, wenn wir kein Geld haben? Wenn wir weiblich sind, oder arbeitslos oder doch Kinder haben? « Dass die aus dem Win-win-Tausch - der Markt generiert Gewinn, Diversität wird im Gegenzug sichtbar - ausgeschlossenen Menschen auch Teil des »falschsexuellen « Wir sind, wird durch die durchgängige Verwendung von Personalpronomen wie »Wir« und »Uns« deutlich gemacht. Das »Wir« der »falschsexuellen« Gemeinschaft, das in dieser Argumentation hergestellt wird, geht nicht in den Anrufungen des Marktes auf. Der - die 
Erörterung des »Für« und »Wider« kommodifizierbarer Diversität - abschließende Satz fügt sich nicht ganz logisch in die Argumentation ein: »Deshalb verteilt man auch mit Werbung bedruckte Kondome, nicht Dental Dams. Unklar bleibt, was die Verteilung von Kondomen statt Dental Dams auf der Pride mit der Tatsache zu tun hat, dass ein »Tausch« mit dem »Markt«Ausschlüsse produziert. Dieser Satz markiert eine Leerstelle in der Argumentation und hat wohl eher die Funktion, wieder zum Thema zurückzuführen. Wurde bisher nur bejaht, dass Diversität vom Markt vereinnahmt wird, wird in diesen Argumentationen implizit auch und ohne Begründung bejaht, dass auch die Pride vom Markt vereinnahmt ist.

Im Folgenden wird dann erneut in Form eines dialogischen Einwand-AntwortFormats der Einwand formuliert, dass »böse Kapitalist*innen« ja überall als »Trittbrettfahrer ${ }^{15}$ mit dabei seien. In diesem Einwand, von dem sich der Text erneut durch Verwendung des Konjunktivs distanziert, werden kapitalistische Strukturen personalisiert. »Böse Kapitalist*innen« und »kapitalistische Trittbrettfahrer« werden als Normalität definiert, die hingenommen werden müsse. Durch die Herstellung einer Analogie zum Fußballspiel, bei dem auch »Bier« und »Würste« verkauft würden, wird diese Normalität beispielhaft illustriert. In dieser Analogie bleibt unsichtbar, worüber nicht gesprochen wird. Beispielsweise die Unterschiede zwischen einem zutiefst kommerzialisierten Fußballspiel, das zumindest offiziell keinen politischen Anspruch hat, und einem Demonstrationszug von Menschen, die sich mehr oder weniger an den Rändern »des Normalen« bewegen. Der Einwand »Kapitalist*innen seien doch eh überall dabei« wird in der weiteren Argumentation nur teilweise entkräftet, indem er gleichzeitig angenommen und relativiert wird. Das eine (kapitalistische »Trittbrettfahrer«) - so das Argument - schließt das andere (das Politische) nicht aus. Hier wird wieder Bezug auf die Kritik »vieler« genommen, die der Pride vorwerfen, nur noch kommerziell und nicht mehr politisch zu sein. Der Text positioniert sich nun zu der Kritik der Community, indem diese gleichzeitig bejaht und abgelehnt wird. Bejaht wird, dass die Pride kommerziell ist, bejaht wird auch der Wunsch nach einer politischen Pride. Abgelehnt wird aber, dass beides einander ausschließt. Dabei rekurriert die Argumentation auf die Fähigkeit von Körpern, auf der Straße gleichzeitig Werbeflyer in die Tasche zu stecken und politische Transparente zu halten: «Und wenn ich all die Kondome und Nivea-Gratismüsterchen in die Tasche stecke, habe ich ja immer noch genug Hände frei, um ein Transparent mit einer politischen Botschaft $\mathrm{zu}$ halten.«Um die mögliche Gleichzeitigkeit von Politik und Kommerz auf der Pride als eine »wahre« Aussage herzustellen, wird ein gemeinsam geteiltes Sprachbild aufgerufen: ein Bild von Körpern auf der Pride, die Transparente hochhalten und Werbung in der Tasche haben. In die Körper können sich - so die hier implizite Argumentation - gleichzeitig eine warenförmige Subjektivierung und eine politische Subjektivierung einschreiben. Damit ist auch die Pride - als ein durch die Warenwelt strukturierter Raum - noch immer ein Ort des Politischen. Sie kann gleichzeitig durch die Warenwelt strukturiert und »Erscheinungsraum ${ }^{16}$ für Körper sein, die »Transparente«

15 Der Begriff »Trittbrettfahrer ist in der maskulinen Form geschrieben, während im restlichen Text der Unterstrich verwendet wird.

16 J. Butler: Anmerkungen zu einer performativen Theorie der Versammlung, S. 117. 
hochhalten. Als ein Erscheinungsraum, der diese Gleichzeitigkeit ermöglicht, kann die Pride auch ein »Umzug für die Falschsexuellen« sein. Die Milchjugend konstituiert sich in diesem Abschnitt als ein Wir, das auf der einen Seite mehr ist als das, was für die Warenwelt interessant ist, auf der anderen Seite ist sie eine Gemeinschaft, die die Pride - obwohl sie durch diese Warenwelt strukturiert ist - bejaht. Bejaht wird die Pride, weil die Milchjugend über die körperlichen Fähigkeiten verfügt, gleichzeitig Werbeflyer einzustecken und Transparente hochzuhalten.

Im vierten Abschnitt werden weitere Zweifel an der Pride als Ort des Politischen geäußert, indem gefragt wird, ob eine »Hypersichtbarkeit « an einem einzigen Tag im Jahr sinnvoll sei oder ob es nicht generell, zu jeder Zeit und an jedem Ort, mehr Sichtbarkeit geben sollte. Hinzu komme, dass das Auftreten von »schrill gekleideten« Menschen auf der Pride abschreckend wirken kann beziehungsweise Schaulustige anzieht.

Nachdem die Möglichkeit einer Gleichzeitigkeit von Politischem und Kommerzialisierung auf der Pride im vorherigen Abschnitt bejaht wurde, wird nun in Form von drei rhetorischen Fragen das Politische der Pride in Frage gestellt: Ist »Hypersichtbarkeit« an einem »einzigen Tag« »sinnvoll«? Sollten nicht alle jeden Tag an unterschiedlichen Orten »sichtbar « sein? Verlieren Menschen in Anbetracht einer »schrill« gekleideten Masse ihre »Berührungsängste« oder werden sie $\mathrm{zu} » S c h a u l u s t i g e n «$, die lediglich ein »Spektakel« betrachten? Körper sind dazu in der Lage, Transparente hochzuhalten und Werbung in die Tasche zu stecken, aber, so die Frage, die hier gestellt wird, ist es überhaupt sinnvoll, politische Transparente in bestimmten Kontexten (einmal im Jahr, Hypersichtbarkeit, schrilles Auftreten) hochzuhalten? Indem das Politische der Pride in Frage gestellt wird, wird es gleichzeitig mit Bedeutungen gefüllt. Das Politische heißt, einen Platz im Feld des Sichtbaren einzufordern, und das immer und überall und nicht nur einmal im Jahr auf der Straße. Politisch ist es aber auch, Berührungsängste abzubauen und nicht als die Anderen angerufen zu werden. Hier werden in Bezug auf die Pride bereits bestimmte Vorstellungen des Politischen - über die die Milchjugend sich als ein widerständiges Wir konstituiert - festgeschrieben.

Im fünften Abschnitt wird zunächst festgehalten, dass es wichtig sei, über all diese Kritiken an der Pride nachzudenken. Dennoch wäre es falsch zu sagen, die Pride sei nicht politisch: Es gebe jedes Jahr ein Motto, es gebe politische Reden und viele Organisationen hätten Forderungskataloge erstellt. Auch wenn diese Dinge nicht immer im Sinne der Milchjugend seien, könne ihnen das Politische nicht abgesprochen werden: Selbst der Party-Truck und die »übersexualisierte Atmosphäre« hätten politische Aspekte.

In diesem Abschnitt wird mit dem zu Beginn durch die affektive Ambivalenz angerufenen »denkenden Subjekt« teilweise gebrochen: »Sicher ist es wichtig, nach der ersten Euphorie über diese Dinge nachzudenken«. Doch andererseits werde mensch mit der Pride »nicht so schnell fertig«. Das Adverb »andererseits« verweist darauf, dass es noch eine andere Seite gibt, die durch das »denkende Subjekt « nicht einholbar ist. Im darauffolgenden Satz wird deutlich, was das »denkende Subjekt « vermeintlich nicht einholen kann: »Zunächst ist sie gar nicht so frei von politischen Forderungen, wie oft behauptet wird.«Das »denkende Subjekt« erkennt dieser Argumentation zu Folge nur, dass der Pride eindeutig jegliches politische Potential fehle. Auch hier grenzt sich die 
Milchjugend erneut implizit von jenen unbestimmten »vielen«ab, für die es nur ein Entweder-oder - "politisch« oder »kommerzialisiert« - gibt. Mit der darauffolgenden ironischen Aussage wird die Position, die Pride sei nur kommerziell, endgültig verworfen: "Es ist keineswegs so, dass sich an der Pride alle an den Händen halten und sich gemeinsam darüber freuen, dass in der Werbung immer mehr gleichgeschlechtliche Paare auftauchen."Wurde vorher nur die mit den Fähigkeiten der Körper verbundene Möglichkeit des Politischen der Pride bejaht, das Politische der Pride aber noch hinterfragt, wird nun im Folgenden das Politische der Pride endgültig als »Wahrheit« hergestellt: »jede Pride hat ein »Motto«, »eine Handvoll Reden« und »Kataloge mit Forderungen«.

In den weiteren Ausführungen findet darüber hinaus eine Abgrenzung zu einem Verständnis des Politischen statt, das auf die »Form von zehnseitigen Argumentarien« begrenzt ist: Selbst der »Party-Truck« ist insofern politisch, als die »Straße eingenommen wird «, und auch die »körperlich-übersexualisierte« Atmosphäre kann ein Statement gegen heteronormative Vorurteile sein. Damit wird die Pride in dem spezifischen Verständnis dessen, was für die Milchjugend politisch ist, als politisch bejaht. Auch Körper auf der Straße an sich oder »Überinszenierungen« als Strategie sind politisch. Die Pride wird in dieser Argumentation insofern als politischer Ort definiert, als sie, mit Butler gesprochen, »Erscheinungsraum « des Politischen ist. ${ }^{17}$ Es gibt Raum für politische Argumente und Forderungen, die Straße wird von Körpern eingenommen, die sonst nicht derart sichtbar sind oder deren Erscheinen auf der Straße sogar gefährlich sein kann. Als Mittel und Zweck der Politik legen Körper auf der Pride zudem offen, was als nicht normal gilt; sie machen so andere Möglichkeiten des Seins denkbar und fordern das Recht ein, als ebendiese Körper ungefährdet auf der Straße erscheinen zu dürfen.

Im sechsten Abschnitt wird zunächst wieder auf die in der Einleitung erwähnte Euphorie Bezug genommen: Die anfängliche Euphorie sei insofern nicht unberechtigt, als die Pride ein Raum ist, an dem sich die Normalität umkehren kann. Gemeinsam gegen Heteronormativität anzukämpfen, mache einen Unterschied, so das abschließende Fazit.

In diesem letzten Abschnitt wird die affektive Anrufung der Pride - Teil einer Bewegung zu sein « - abschließend bejaht. Die Euphorie ist als Effekt von Erfahrungen, in denen "Normalität sich plötzlich umgekehrt hat « und »sich Falschheit unter Falschen plötzlich gut anfühlt«, legitim. Ihr wird Legitimität zugesprochen, weil beziehungsweise wenn sie ein Effekt des Politischen der Pride ist - und nicht ein der »zarten Jugend geschuldeter Anfänger*innenfehler«. Hier wird unterschieden zwischen einer »naiven« Bejahung der affektiven Anrufung und einer Bejahung der Euphorie, gerade weil sie mit dem Politischen der Pride verknüpft ist. Die positive affektive Anrufung der Pride wird von der Milchjugend nicht in ihrer Unmittelbarkeit bejaht, sondern erst, nachdem sie verstanden wurde. Erst nachdem sie der Prüfung - in der das politische Potential Kriterium für »richtig « und »falsch « ist - unterzogen wurde, wird die Euphorie als affektive Anrufung angenommen. Abschließend wird zudem das notwendig kollektive Moment des Politischen hervorgehoben: »Es macht doch einen Unterschied, ob man ganz alleine 
gegen die Heteronormativität anrennt oder ob man dazu die Unterstützung einer ganzen Masse hat.« Hier findet sich in der Argumentation eine Leerstelle. Es bleibt offen, warum und inwiefern es einen Unterschied macht, "gemeinsam« $\mathrm{zu} »$ rennen«. Diese Leerstelle wird vermeintlich gefüllt, indem implizit erneut das Bild von »Körpern auf der Straße« aufgerufen wird. Es ist das Bild von Körpermassen, die auf der Pride gegen Normen anrennen. Über dieses Bild wird der Körper erneut als Mittel der Politik festgeschrieben.

Im letzten Satz des Artikels wird der "Queer Nation«-Slogan »We're queer, we're here, get used to it! « zitiert. Damit wird in der Auslotung des Verhältnisses der Milchjugend zur Pride abschließend ein Bezug zu queeren Politiken, konkret zur »Queer Nation«, hergestellt. "Queer Nation« gilt als erste politische Gruppe, die sich - in dem auf der Pride verteilten »Queer Nation Manifesto« - den Begriff »queer « angeeignet hat. ${ }^{18} \mathrm{Da}$ mit positioniert sich die Milchjugend in einer queeren Bewegung, die Ja sagt zur Pride als Ort, an dem - dadurch, dass queere Körper auf der Straße erscheinen - das Recht, dort $\mathrm{zu}$ erscheinen, geltend gemacht wird. Die queeren Körper der Milchjugend nehmen sich das Recht, auf die Straße zu gehen: »We are queer, we are here«; die anderen sollen sich daran gewöhnen: "get used to it!«. In und über die Community hinaus versinnbildlicht »Queer Nation« queere Politiken par excellence. Auch wenn es nicht explizit ausgesprochen wird, beantwortet dieser »Milchbüechli«-Artikel die Frage, ob die Pride »noch ein Umzug für die Falschsexuellen ist «, eindeutig: Die Pride ist ein Erscheinungsraum des Politischen und deshalb sagt die Milchjugend Ja zur euphorischen Anrufung ebendieser. Damit grenzt sie sich von den »vielen« aus der Community ab, die die Pride als Ort des Politischen verwerfen.

\subsubsection{Der eigentliche CSD}

LesMigraS nutzt Pressemitteilungen und Redebeiträge vor allem, um - anlässlich verschiedener Erinnerungstage oder Demonstrationen - Rassismus und Transphobie zu thematisieren. Der CSD als eine Erinnerungsveranstaltung, an der LesMigraS meistens teilnimmt, ist besonders häufig Anlass und auch Gegenstand von Statements dieser Einrichtung. Im Analysezeitraum wurden drei Pressemitteilungen und drei Redebeiträge anlässlich des CSD veröffentlicht. Die erste Pressemitteilung bezieht sich auf Judith Butlers Ablehnung des - vom Berliner CSD e.V. jährlich vergebenen - ZivilcouragePreises im Jahr 2010. Dieses Ereignis, das über Berlin hinaus, auch international, diskutiert wurde, löste eine Debatte um Rassismus in vielen Teilen der queeren deutschsprachigen Communitys aus. In ihrer Rede betonte Butler explizit die "Courage« von Einrichtungen wie »Gladt e.V.«, LesMigraS, »SUSPECT« und »Reach Out « und wirft dem Berliner CSD e.V. eine »Kompliz*innenschaft mit Rassismus«, insbesondere mit »antimuslimischen Rassismus« vor. In diesem 2010 von LesMigraS veröffentlichten Statement bedankt sich die Einrichtung bei Judith Butler für die »deutliche Stellungnahme 
gegen Rassismus «. ${ }^{19}$ LesMigraS war eine der Einrichtungen, die im Vorfeld der Preisvergabe mit Judith Butler im Austausch standen und sie auf den Rassismus am Berliner CSD aufmerksam machten. Die Einrichtung selbst nimmt seit 2009 nicht mehr am Berliner CSD teil. ${ }^{20}$ Meistens ist das Projekt aber auf dem alternativen Transgenialen CSD vertreten, der 1998 in Abgrenzung zum Rassismus und zur Kommerzialisierung des Berliner CSD entstand. ${ }^{21}$ In den Jahren 2012 und 2013 hielt LesMigraS jeweils eine Rede auf dem Transgenialen CSD. In beiden Reden nimmt die Einrichtung Bezug auf das jeweilige Motto »antifaschistisch, queerfeministisch, antirassistisch, solidarisch ${ }^{22}$ beziehungsweise »Solidarisch Queer-Topia ${ }^{23}$ und füllt es selbst mit Bedeutung. Beide Male spricht LesMigraS vor allem darüber, wie ein solidarischer CSD aussehen sollte. Ein Jahr später veröffentlicht die Einrichtung zunächst das Statement »Politik ist mehr als ein Name. Statement zur Umbenennung des Berliner CSD in Stonewall ${ }^{24}$ In der Stellungnahme kritisiert LesMigraS die Aneignung der Bezeichnung "Stonewall« durch den Berliner CSD: Solange dieser nicht auch politisch an die Geschichte von Kämpfen gegen Rassismus, Transphobie, Homophobie und Polizeigewalt anknüpft, soll der Name nicht vereinnahmt werden. ${ }^{25}$ Der Versuch des Berliner CSD e.V., den Namen der Berliner CSD-Parade in »Stonewall Berlin« umzuändern, hat in der ganzen Berliner Community zu heftigen Debatten geführt. Aus diesen Auseinandersetzungen formierte sich unter anderem das CSD-Aktionsbündnis, das 2014 eine eigene, dritte Parade organisiert hat. In diesem Zeitraum wurde auch das für die Feinanalyse ausgewählte Diskursfragment "Mehrdimensionales Empowerment statt eindimensionaler Feiertag ${ }^{26}{ }^{26}$ veröffentlicht. Nachdem LesMigraS im Jahr 2014 an keiner der drei Paraden teilgenommen hat, nimmt die Einrichtung 2015 am Kreuzberger CSD $^{27}$ teil und hält dort auch eine Rede, in der Be-

19 LesMigraS: Klares Signal gegen Rassismus. Judith Butler lehnt Zivilcourage-Preis des Berliner-CSD e.V. ab. 2010, https://lesmigras.de/tl_files/lesmigras/pressemitteilungen/Stellungnahme_LB_Butler .pdf vom 26.09.2020.

20 Die Siegessäule: CSD in Berlin. Konflikt und Konsens: Die Siegessäule-Podiumsdiskussion 2014, https://www.siegessaeule.de/magazin/1011-konflikt-und-konsens-die-siegess\%C3\%A4ule-po diumsdiskussion/ vom 20.10.2020.

21 Als »Berliner « oder auch »großer« oder offizieller CSD wird die ursprüngliche Parade bezeichnet. Bereits 1998 ist in Abgrenzung zum Rassismus und zur Kommerzialisierung in Berlin der Transgeniale CSD (TCSD) entstanden, der dann ab 2014 zum Kreuzberger CSD (KCSD) wurde. Das CSDAktionsbündnis hat 2014 eine dritte Parade organisiert.

LesMigraS: Redebeitrag LesMigraS auf TCSD 2012, https://lesmigras.de/tl_files/lesmigras/pressem itteilungen/Redebeitrag_LesMigraS_TCSD_2012.pdf vom 20.11.2019.

23 LesMigraS: Rede/Audiobeitrag von LesMigraS auf dem TCSD 2013, https://lesmigras.de/tl_files/les migras/pressemitteilungen/Redebeitrag_LesMigraS_TCSD_2013.pdf vom 20.11.2020.

24 LesMigraS: Politik ist mehr als ein Name. Statement zur Umbenennung des Berliner CSD in Stonewall 2014, https://lesmigras.de/tl_files/lesmigras/pressemitteilungen/Statement_CSD_Stonewall_2 014.pdf vom 20.11.2020.

25 Symbolisch scheint der Begriff Stonewall noch enger mit den Kämpfen im Juni 1969 verknüpft zu sein als die Christopher Street, in der die Bar stand und die Kämpfe stattgefunden haben.

26 LesMigraS: Pressemitteilung anlässlich des Christopher Street Days.

27 Der Transgeniale CSD hat 2013 das letzte Mal stattgefunden. Der Kreuzberger CSD ist seit 2014 der alternative CSD in Berlin. 
zug genommen wird auf das Motto des Kreuzberger CSD »unanständig, unintegriert, unbequem gegen Krieg, Rassismus und Ausbeutung. Queer bleibt Radikal«. ${ }^{28}$

Die Pressemitteilungen und Redebeiträge von LesMigraS - so auch das für die Feinanalyse ausgewählte Diskursfragment »Mehrdimensionales Empowerment statt eindimensionaler Feiertag « - sind auf der projekteigenen Homepage in der Rubrik »Archiv ${ }^{29}$ downloadbar. ${ }^{30}$ Der Teaser beinhaltet jeweils die Überschrift der Pressemitteilung beziehungsweise des Redebeitrags und den ersten Absatz der Stellungnahme oder eine kurze Zusammenfassung. Darunter befindet sich ein Link, über den jeweils der vollständige Textbeitrag als PDF downloadbar ist. Formal sind die meisten Pressemitteilungen und Redebeiträge von LesMigraS ähnlich aufgebaut. So auch das für die Feinanalyse ausgewählte Diskursfragment: Oben in der Kopfzeile befindet sich auf der linken Seite das Logo der »Lesbenberatung Berlin e.V.«, darunter die Adresse, Homepage und Telefonnummer. Rechts daneben stehen das Logo von LesMigraS, die Bezeichnung des Arbeitsbereiches (Antidiskriminierungs- und Antigewaltbereich der Lesbenberatung Berlin e.V.), die Homepageadresse und die Telefonnummer. Darunter befindet sich fett und mittig ausgerichtet die Überschrift, die zumeist den Anlass und die Textsorte beschreibt: »Pressemitteilung anlässlich des Christopher Street Days«. Zielgruppe des ausgewählten Diskursfragmentes ist in diesem Fall die LGBTIQ-Community in Berlin, aber auch die eigene Gemeinschaft. Im Gegensatz zu den meisten anderen Stellungnahmen von LesMigraS befinden sich unter diesem Statement ein Name und eine Telefonnummer für Interviewanfragen. Das verweist - wie auch die Deklarierung als Pressemitteilung - auf das Ziel, eine über die Community hinausgehende mediale Aufmerksamkeit erlangen zu wollen. Unter der ersten Überschrift steht ebenfalls fett und mittig ausgerichtet der Titel, der zugleich das zentrale Statement dieser Pressemitteilung darstellt: »Mehrdimensionales Empowerment statt eindimensionaler Feiertag«. Eine solche Komprimierung ist typisch für Textsorten, deren primäres Ziel es ist, wichtige Informationen schnell und auf einen Blick zu vermitteln. Die zu analysierende Pressemitteilung umfasst etwas mehr als eine Seite und ist in sechs kurze, übersichtlich voneinander getrennte Absätze unterteilt. Formal gesehen erfüllt das Diskursfragment die gängigen Anforderungen einer Pressemitteilung. Sie ist kurz und übersichtlich strukturiert. Gleich im ersten Absatz wird die zentrale Aussage - dass LesMigraS an keinem CSD teilnimmt - abgegeben. In den folgenden Absätzen werden die Gründe erläutert.

Im ersten Abschnitt wird erklärt, dass der CSD für »politische und gesellschaftliche Kämpfe« von LSBTIQ-Menschen stehe und dass LesMigraS an keiner der drei in Berlin stattfindenden Paraden teilnehmen werde.

28 LesMigraS: Redebeitrag von LesMigraS auf dem KCSD 2015, https://lesmigras.de/tl_files/lesmigras /pressemitteilungen/Redebeitrag_LesMigraS_KCSD_2015.pdf vom 20.11.2020.

29 LesMigraS: Archiv.

30 Ob Pressemitteilungen und Stellungnahmen aktiv an Journalist*innen weitergeleitet werden, ist nicht bekannt. 
LesMigraS legt gleich zu Beginn ein vermeintliches Paradoxon offen. Es besteht ein Widerspruch zwischen dem, was der CSD, der Einrichtung zufolge, »eigentlich« ist und dem, was der CSD aktuell ist. Zunächst schreibt LesMigraS fest, was der CSD eigentlich ist: eine Parade »für die politischen und gesellschaftlichen Kämpfe von Lesben, Schwulen, Bisexuellen, Trans*, Inter* und Queers«. Mit dem Begriff des Kampfes wird implizit auf die im Jahr 1969 in New York stattgefundenen Proteste Bezug genommen, an die der CSD erinnern soll. Gleichzeitig stellt der Begriff klar, dass der CSD kein Ort für »Kuschelpolitiken« oder Kommerzialisierung, sondern für politische und gesellschaftliche Auseinandersetzungen ist. Gesellschaftliche und politische Kämpfe werden als das Eigentliche des CSD festgeschrieben (und damit gleichzeitig gefordert). Sich durch eine Passivkonstruktion distanzierend, legt LesMigraS dann einen Widerspruch offen zwischen dem, was der CSD der Einrichtung zufolge eigentlich ist - Ort des politischen Kampfes - , und dem, was tatsächlich auf dem CSD »häufig « passiert: Der CSD wird "weltweit häufig als queerer Feiertag« zelebriert. Im Anschluss daran erklärt LesMigraS, dass die Einrichtung an den diesjährigen CSD-Paraden in Berlin nicht teilnehmen werde: »An keinem der Events wird sich LesMigraS und die Lesbenberatung Berlin beteiligen.«Der Zusammenhang zwischen dem Widerspruch und der Nicht-Teilnahme wird nicht explizit, sehr wohl aber durch die Anordnung des Textes hergestellt. Die Lücke zwischen dem, was der CSD eigentlich ist - Ort des politischen Kampfes -, und dem, was der CSD tatsächlich ist - queerer Feiertag - , hat etwas damit zu tun, dass LesMigraS in diesem Jahr an keiner der drei CSD-Paraden in Berlin teilnimmt. Die Kritik am CSD findet im Namen eines eigentlichen CSD statt. ${ }^{31}$ Dieses Verfahren ist auch schon im Artikel der Milchjugend zu finden, die ihr Unbehagen über die Diskrepanz zwischen dem, was der CSD eigentlich sein sollte (Ort für politische Botschaften), und dem, was tatsächlich passiert (nackte Muskeltypen, die tanzen), erläutert. Die Unterscheidung zwischen dem, was der CSD eigentlich ist und sein sollte, und dem, was der CSD ist, spielt in der gesamten Pressemitteilung eine zentrale Rolle.

Im zweiten Abschnitt schreibt LesMigraS, dass die Einrichtung sich im Vorfeld in die Auseinandersetzungen um den CSD eingebracht habe. LesMigraS habe dort gefordert, dass es um gemeinsame Kämpfe gegen »Klassismus, Rassismus, Trans*Diskriminierung, Inter*-Diskriminierung und Homophobie« gehen soll. Das würde bedeuten, sich nicht nur vor Ort diesen Kämpfen zu widmen, sondern auch über den einen Tag hinaus.

LesMigraS begründet in diesem Abschnitt, warum der CSD in diesem Jahr keine Parade sei, an der LesMigraS »einen Grund zum Jubeln ${ }^{32}$ hat. Zunächst wird der CSD (in Berlin) als ein generell umkämpfter Ort ausgewiesen: »Es gab viele Auseinandersetzungen rund um alle Veranstaltungen am CSD.« LesMigraS positioniert sich als Gruppe, die an den Debatten um den CSD teilhat, und versucht, eigene Positionen einzubringen. Erneut folgen Bestimmungen dessen, was der CSD eigentlich sein sollte: Es »muss [...]

31 Auch Foucault spricht von einer Kritik im Namen dessen, was eigentlich ist, als einem gängigen Verfahren, das sich beispielsweise in der Kritik des Kynismus, aber auch des Sozialismus zeigt. M. Foucault: Der Mut zur Wahrheit, S. 267. 
um ein gemeinsames Kämpfen gegen Rassismus, Klassismus, Trans*-Diskriminierung, Inter*-Diskriminierung und Homophobie gehen.« Das Verb »muss« verweist auf eine Bedingung, eine Notwendigkeit, an der der CSD nicht vorbei kann, will er LesMigraS einschließen. Es ist eine Setzung. Ein Konjunktiv, gekoppelt mit einer Wenn-dannKonstruktion im darauffolgenden Satz, verweist darauf, dass der CSD diese Bedingung nicht erfüllt: »Wenn dies wirklich im Mittelpunkt stehen würde, dann würde ein mehrdimensionales und gesellschaftskritisches Engagement für ein selbstbestimmtes Leben ohne Gewalt und Diskriminierung, auch über diesen Tag hinaus, sichtbar werden.« Erneut wird die Diskrepanz zwischen dem, was der CSD eigentlich sein sollte, und dem, was tatsächlich dort passiert, offengelegt. Ein »mehrdimensionales und gesellschaftskritisches Engagement «, das als Bedingung für den CSD konkretisiert wird, ist nicht sichtbar.

Die Bestimmungen dessen, was vom CSD gefordert wird, ist gleichzeitig sehr offen und sehr dicht. Zunächst einmal wird der CSD über den Begriff des »Engagements« angerufen. Der Begriff enthält ein stark »subjektives Moment, eine ethische Komponente ${ }^{33}$ Menschen engagieren sich unbezahlt für eine gute Sache, hinter der sie stehen. ${ }^{34}$ Ein "guter« Mensch zu sein, heißt, sich für die »richtige« Sache zu engagieren. Über die Adjektive »mehrdimensional« und »gesellschaftskritisch« wird das Spezifische des Engagements - das, was das Engagement zu einer "guten« Sache macht näher bestimmt. Allerdings wird keiner der beiden Begriffe erläutert. Bezug nehmend auf ein Wissen um die Arbeitsschwerpunkte von LesMigraS, aber auch die Bestimmungen dessen, was der CSD eigentlich sein sollte, bedeutet mehrdimensional, dass Mehrfachdiskriminierungserfahrungen in den Blick genommen werden. Das mehrdimensionale Engagement - oder auch Empowerment, wie es in der Überschrift heißt - ist aber auch der Gegensatz zum »eindimensionalen Feiertag«. Im Unterschied zu »eindimensional« kann »mehrdimensional« so auch bedeuten, sich »über diesen Tag hinaus « $\mathrm{zu}$ engagieren. LesMigraS fordert also einen intersektionalen CSD, in dem es um politische Auseinandersetzungen, über den CSD hinaus, geht. Auch der Begriff "gesellschaftskritisch« wird nicht näher erläutert. Allerdings verweist die Forderung nach einem "gesellschaftskritischen « CSD darauf, dass das, was der CSD macht, eben nicht gesellschaftskritisch ist. LesMigraS verwendet mit "gesellschaftskritisch « einen Begriff, der mit Ernesto Laclau als »leerer Signifikant« bezeichnet werden kann. Laclau zufolge gibt es leere Signifikanten, »weil, jedes Signifikationssystem um einen leeren Platz herum konstruiert ist, der aus der Unmöglichkeit resultiert, ein Objekt zu produzieren, welches die Systemhaftigkeit des Systems trotz alledem erfordert «. ${ }^{35}$ Der leere Signifikant verweist positiv auf diese Unmöglichkeit. In seiner Positivität und Unmöglichkeit ist es der Ort, von dem aus die »Grenzen der Bezeichnung« formuliert werden, indem die Differenzen des Bezeichnungssystems ausgelöscht werden. Indem der leere Signifikant die »differentielle Natur eines Bezeichnungssystems entleert«, kann eine Totalität

33 Hans ]. Sandkühler/Arnim Regenbogen/Chup Friemert: Europäische Enzyklopädie zu Philosophie und Wissenschaften, Hamburg: Meiner 1990, S. $696 \mathrm{f}$.

34 Nicht nur LesMigraS, sondern auch andere Projekte (vor allem die Villa und das Jugendnetzwerk Lambda BB) sprechen sehr häufig von Engagement. 
bezeichnet werden, die konstitutiv unerreichbar ist. ${ }^{36}$ Vor allem die Bezeichnung "gesellschaftskritisch « entleert bestehende Differenzen in Bezug auf die Frage, was gesellschaftskritisch ist. Es wird eine Einheit hergestellt, die sich auf ein gemeinsames Ziel hinbewegt, zu dem der CSD nicht Nein sagen kann: ein gesellschaftskritisches Engagement. Indem nicht expliziert wird, welche Bedeutungen die Forderungen nach einem mehrdimensionalen und gesellschaftskritischen Engagement haben, werden bestehende Differenzen bewusst ignoriert. Stattdessen werden diese Begriffe implizit in dem Verständnis, das LesMigraS von ihnen hat, als eindeutig gesetzt und dadurch als etwas Selbstverständliches eingefordert.

Im Anschluss wird das Zukünftige, in dessen Dienst sich das "mehrdimensionale« und "gesellschaftskritische« Engagement stellen soll, festgeschrieben: »für ein selbstbestimmtes Leben ohne Gewalt und Diskriminierung «. ${ }^{37}$ Hier wird bewusst eine $\mathrm{Zu}$ künftigkeit formuliert, die konstitutiv unerreichbar ist. Es ist die Formulierung eines idealen Ziels, das mittels mehrdimensionalen, gesellschaftskritischen Engagements erreicht werden soll. Es ist ein Ziel, das sehr offen und somit zwangsläufig ein gemeinsam geteiltes ist. So wird ein gemeinsames Ziel ausgerufen, das der CSD nicht nicht wollen kann. Das, was umstritten ist - nämlich der Weg, der eingeschlagen werden soll, um dieses Ziel zu erreichen -, bleibt unbenannt. So kann der CSD nicht Nein sagen $\mathrm{zu}$ den Forderungen von LesMigraS, gleichzeitig steht hinter diesen Forderungen aber implizit eine bestimmte Vorstellung davon, was es bedeutet, sich auf den Weg zu diesem Ziel zu machen. Diese Vorstellungen werden im Anschluss beispielhaft erläutert. LesMigraS benennt, was sich beispielsweise an den CSD-Praktiken ändern müsste, damit der CSD zu dem wird, was er eigentlich ist: »Es würde sich dann an diesem Tag Kämpfen vor Ort, wie z.B. gegen die diskriminierende Lebenssituation von geflüchteten LSBTIQ-Menschen oder gegen Rassismus- und Klassismuserfahrungen von LSBTIQ of Color, angeschlossen werden, statt sich entwicklungspolitisch $\mathrm{zu}$ anderen Ländern $\mathrm{zu}$ äußern.« Mit dieser konkreten Bestimmung dessen, was LesMigraS vom CSD fordert, wird Bezug auf eine postkoloniale Kritik genommen. Anstatt selbst durch entwicklungspolitische Äußerungen Rassismen zu produzieren, soll sich der CSD Kämpfen gegen Rassismus im eigenen Land anschließen. Erneut wird Kritik am CSD - »er ist rassistisch « - im Namen dessen, was er eigentlich sein sollte - "antirassistisch « geübt. Dabei wird an eine ganze Debatte postkolonialer Kritiken des Othering angeschlossen, die als bekannt vorausgesetzt wird.

LesMigraS fordert einen CSD, der so ist, wie ein CSD eigentlich ist, also sein sollte, und nicht so, wie er tatsächlich ist. Dabei wird das, was der CSD eigentlich ist, von LesMigraS gesetzt und gleichzeitig eingefordert, indem es den Namen einer großen Utopie bekommt - »Gewalt- und Diskriminierungsfreiheit« - , zu der der CSD nicht Nein sagen kann. Die Lücke zwischen dem eigentlichen und dem aktuellen CSD, die

36 Ebd., S. $69 f$.

37 Wenn LesMigraS von einem »selbstbestimmten« Leben spricht, ist nicht ein individualistisches Selbst gemeint. Selbstbestimmung ist vielmehr ein Begriff, der im Kontext der Antigewalt- und Antidiskriminierungsarbeit für das Recht steht, selbst den passenden Weg für den Umgang mit dem Erlebten zu finden. Der Begriff ist vor allem auch in Abgrenzung zum Paternalismus in der sozialen Arbeit, aber auch zu einem eurozentrischen Paternalismus zu verstehen. 
auch der Grund für die Nicht-Teilnahme von LesMigraS ist, wird dann näher bestimmt: Der CSD sollte antirassistisch und nicht das Gegenteil, nämlich rassistisch, sein.

Im dritten Abschnitt kritisiert LesMigraS, dass in diesem Jahr viele Gruppen an Planungs- und Entscheidungsprozessen um den CSD ausgeschlossen worden seien. LesMigraS hätte sich gewünscht, dass alle Gruppen aktiv eingeladen worden wären und dass es eine echte Bereitschaft für Veränderungen gegeben hätte.

Dieser Abschnitt wird mit einem »Stattdessen« eingeführt. Das »Stattdessen« bezieht sich weiterhin auf die Diskrepanz zwischen dem, was der CSD eigentlich sein sollte, und dem, was »häufig passiert«: »Stattdessen mussten wir aber feststellen, dass nicht alle in der Community aktiv in verschiedene Entscheidungsprozesse einbezogen wurden, sondern viele Planungen ohne Transparenz oder Offenheit stattfanden und erst relativ spät öffentlich oder halb-öffentlich bekannt gegeben bzw. zur Diskussion gestellt wurden." Ein CSD, wie er eigentlich sein sollte, ist ein CSD, an dem alle die Möglichkeit haben, mitzuentscheiden. Es ist ein offener CSD, der von LesMigraS gefordert wird:»Wir hätten uns [...] ein aktives Einladen und Beteiligen der Communities, in deren Namen sie sprechen, gewünscht«. Das, was stattdessen passiert, ist ein CSD, der diejenigen Gruppen, die er repräsentiert, nicht teilhaben lässt an Entscheidungen darüber, wie der CSD organisiert ist. Der aktuelle CSD spricht - der Argumentation von LesMigraS zufolge - für diejenigen, die nicht in die Entscheidungsprozesse miteinbezogen sind. In dem, was er repräsentiert, schließt er die Gruppen ein, die er jedoch aus den Entscheidungsprozessen darüber, was wie repräsentiert wird, ausschließt. Auch hier knüpft LesMigraS an postkoloniale Debatten an, in denen repräsentationspolitische Fragen - wer für wen in wessen Namen spricht - eine zentrale Rolle spielen. Es wird im Sinne Spivaks eine Lücke zwischen Repräsentation als »sprechen für« und Repräsentation als »darstellen « offengelegt. ${ }^{38}$

Die Forderung nach einem »aktiven Einladen« wird über die Formulierung »Wir hätten uns gewünscht« geäußert. Hier findet eine Verschiebung, von der anfänglich fordernden Haltung hin zu einer sensibleren Kritik, statt. Der CSD wird als eine Gemeinschaft angerufen, zu der eine Beziehung besteht, in der LesMigraS Erwartungen (Wünsche) äußern kann. Durch die Formulierung »Wir hätten uns gewünscht « wird implizit eine Enttäuschung über Erwartungen, die nicht erfüllt wurden, ausgedrückt: Der CSD hat keine »echte Bereitschaft « gezeigt, »rassistischen Strukturen« entgegenzutreten. Hier wird eine affektive Komponente eingebracht, der ähnlich wie der moralischen - ein gewaltfreies und diskriminierungsfreies Leben zu wollen - kaum widersprochen werden kann. Wer kann einem schon die berechtigte Enttäuschung über Erwartungen und Wünsche in einer Beziehung, die einfach ignoriert werden, absprechen? Und wer will schon enttäuschen? Wenn der CSD über diese affektive Ebene der »Enttäuschung« als die Gemeinschaft angerufen wird, die er nicht ist, kann er sich dieser Anrufung kaum entziehen.

Auch in diesem Abschnitt wird die Kritik am CSD, im Namen des eigentlichen CSD, der gleichzeitig gefordert wird, fortgeführt: Der CSD sollte alle »aktiv« einladen, statt Gruppen aus den Entscheidungsprozessen um die Veranstaltung auszuschließen. 
Im vierten Abschnitt wird das Empowerment-Verständnis von LesMigraS expliziert. Empowerment heiße, verschiedene Bedürfnisse zu erfassen und gemeinsam Handlungsstrategien $\mathrm{zu}$ entwickeln, auszuwerten und sich weiter $\mathrm{zu}$ bewegen, indem kritische Auseinandersetzungen gefördert werden - jeden Tag im Jahr.

Ohne dass hervorgeht, wie der Empowerment-Begriff an die vorherige Argumentation anschließt, wird dieser näher bestimmt. Durch die Leerstelle in der Argumentation wird selbstverständlich ein Zusammenhang zwischen dem, was der CSD eigentlich sein sollte, und dem Empowerment-Begriff hergestellt, der nicht hinterfragbar ist. Empowerment ist bisher nur in der Überschrift als Teil des Gegensatzpaars "mehrdimensionales Empowerment « - das was der CSD sein muss und eigentlich ist - versus »eindimensionaler Feiertag « - das was der CSD ist - vorgekommen. Der EmpowermentBegriff ist ein zentraler Bestandteil der Arbeit von LesMigraS. Der Begriff kann auf die Errungenschaften der Schwarzen Bürgerrechtsbewegungen in den USA zurückgeführt werden. Er wurde dort erstmals durch das Werk von Barbara Bryant Solomon mit dem Titel: »Black Empowerment: Social Work in Oppressed Communities « ${ }^{39}$ diskutiert. Mit der Verwendung dieses Begriffs verortet sich LesMigraS in einer Tradition der antirassistischen sozialen Arbeit, die die "powerlessness« von marginalisierten Gruppen als strukturell bedingt versteht, den Fokus der Arbeit aber auf individuelle und kollektive Handlungsstrategien im Umgang mit dieser Machtlosigkeit legt. Empowerment ist zugleich konkrete Praxis und Selbstverständnis von LesMigraS. In der Pressemitteilung folgt nun eine Aufzählung, in der das, was LesMigraS unter Empowerment versteht als wichtige Bestimmung dessen, was der CSD eigentlich sein soll -, näher bestimmt wird:

»In unserem Verständnis geht es bei Empowerment darum, verschiedene Bedürfnisse und Positionen aus den Communities zu erfassen, gemeinsam stärkende Handlungsstrategien zu entwickeln, diese zusammen möglichst diskriminierungssensibel umzusetzen, im Anschluss auszuwerten und sich gemeinsam zunehmend weiter zu bewegen, Kritik einzuladen und Auseinandersetzungen als wichtigen Bestandteil von Bewegungen willkommen zu heißen.«

LesMigraS konkretisiert über die Bestimmung des Empowerment-Begriffs die Forderungen an den CSD. Erneut werden diese Bestimmungen in einen Gegensatz zu dem, was der CSD aktuell ist - eindimensionaler Feiertag -, gestellt: »Ein solches mehrdimensionales Empowerment kann nicht an einem Tag im Jahr passieren, sondern braucht ein ganzjährliches [sic!] Engagement.«

Der CSD, den LesMigraS fordert, soll - ähnlich wie LesMigraS selbst - um den Empowerment-Begriff angeordnet sein. Gleichzeitig übt die Einrichtung Kritik an einem CSD, der sich nur an einem Tag im Jahr engagiert.

Im fünften Abschnitt wird zusammenfassend darauf verwiesen, dass ein Austausch über »Empowerment «, »Sensibilität « und »gesellschaftliche Veränderungen« wichtig sei und dass das in den Diskussionen zum CSD in diesem Jahr nicht funktioniert habe. Es 
brauche dringend eine Veränderung, so dass People of Color, behinderte Menschen und Menschen mit Klassismuserfahrungen im Mittelpunkt der Arbeit stehen.

In diesem Abschnitt setzt LesMigraS sich als Teil des CSD, indem das erste Mal in der Wir-Form darüber gesprochen wird, was passieren muss, damit der CSD zu dem wird, was er der Einrichtung zufolge eigentlich ist: „Wir finden es wichtig, uns auszutauschen «, »[w]ir brauchen dringend einen Perspektivwechsel«. Während LesMigraS in den vorherigen Abschnitten darauf verweist, dass der CSD so, wie er aktuell ist, Gruppen wie LesMigraS ausschließe, wird hier trotzdem im Namen eines gemeinsamen Wir gesprochen. Dieses Wir - das wie vorher beschrieben kein Wir ist - wird gesetzt, um ebendieses Wir einzufordern. Durch das Sprechen von einem gemeinsamen Wir kann in dessen Namen ein gemeinsames Ziel festgeschrieben werden, das gleichzeitig auch eingefordert wird. Mit Judith Butler gesprochen wird ein performativer Widerspruch geltend gemacht: »jemand der nicht autorisiert ist, in dem oder als das Universale zu sprechen, [...] erhebt trotzdem Anspruch « darauf. Es wird aus »der gespaltenen Situation dessen gesprochen, der sowohl autorisiert als auch nicht autorisiert ist. ${ }^{40}$ Indem das Recht in Anspruch genommen wird, im Namen eines gemeinsamen Wir zu sprechen, aus dem LesMigraS ausgeschlossen ist, wird der Einschluss in dieses Wir gefordert. Es wird eine gemeinsame »Vision« eines CSD festgeschrieben, in der sich das Wir über »Empowerment, Diskriminierung, Sensibilität und gesellschaftliche Veränderungen« austauscht, das Wir einen »Perspektivenwechsel« braucht und Menschen mit Mehrfachdiskriminierungserfahrung in den »Mittelpunkt der Politik« gestellt werden. Das Wir wird gesetzt und gleichzeitig eingefordert. Die Lücke zwischen dem, was der CSD eigentlich ist, und dem, was dort tatsächlich passiert, wird geschlossen, indem im Namen eines gemeinsamen Wir gesprochen wird, das durch dieses Sprechen gleichzeitig gesetzt und eingefordert wird. Doch welche Art von Wir wird eingefordert?

Es ist ein Wir, das sich aus der »Sackgasse« dessen, was auf dem CSD aktuell und häufig passiert, hinausbewegt. Durch die Redewendung von der »Sackgasse«, in die sich der CSD hineinmanövriert habe, wird die »Notwendigkeit « einer Veränderung plausibel gemacht. Es wird ein aus dem Straßenverkehr entlehntes Bild - das auch als »Kollektivsymbol« bezeichnet werden kann - aufgerufen. Kollektivsymbole sind ein in einer Gesellschaft existierendes »Repertoire an Bildern «, die ein System bilden, die das Erleben und Deuten der Wirklichkeit strukturieren. ${ }^{41}$ In diesem Fall wird das Bild der Sackgasse verwendet, um die Notwendigkeit einer Veränderung festzuschreiben. Die Sackgasse steht für einen »festgefahrenen « CSD, der sich weder vor- noch zurückbewegt. Es wird plausibel, dass der CSD neue Wege braucht - einen »Perspektivenwechsel«. Der CSD wird über das Bild der »Sackgasse« als etwas Geschlossenes (Ein- und Ausschließendes) festgeschrieben, das an seine Grenzen gekommen ist und das sich notwendigerweise »öffnen« muss (»Perspektivenwechsel«). Ein Wir, das sich im Gegensatz dazu aus dieser »Sackgasse« herausmanövriert, ist ein offenes Wir, das sich über »Empowerment«, »Diskriminierung«, "Sensibilität« und "gesellschaftliche Veränderungen« austauscht. Wie in den vorherigen Abschnitten handelt es sich, mit Ausnahme des EmpowermentBegriffs, um Begriffe, die nicht erläutert werden. Während Diskriminierung und Sen-

40 J. Butler: Haß spricht, S. 145.

41 S. Jäger: Kritische Diskursanalyse, S. 55. 
sibilität, ähnlich wie der Empowerment-Begriff, zentrale Begriffe antirassistischer sozialer Arbeit - und damit auch der Arbeit von LesMigraS - sind, handelt es sich bei der "gesellschaftlichen Veränderung « um eine Bezeichnung, die auch als leerer Signifikant bezeichnet werden kann. Erneut wird die Bedeutungsvielfalt dieses Begriffes bewusst ignoriert und implizit die LesMigraS-Vision von gesellschaftlicher Veränderung als universell eingesetzt. Auch hier wird der Weg aus der »Sackgasse«, also der Weg zur "gesellschaftlichen Veränderung «, konkretisiert und gleichzeitig als das, was der CSD sein soll, festgeschrieben: »Es muss darum gehen, People of Color, behinderte Menschen, Menschen mit Klassismuserfahrungen etc. in den Mittelpunkt unserer politischen Arbeit zu stellen. « Auch hier wird Kritik am CSD im Namen dessen, was der CSD eigentlich sein sollte, formuliert: »statt nur dann sinklusiv< zu sein, wenn es gerade ins Programm passt «.

Indem LesMigraS im Namen eines Wir spricht, zu dem LesMigraS selbst eine gespaltene Position einnimmt, fordert die Einrichtung im Sprechen ein Wir, an dem LesMigraS teilhat.

Der sechste Abschnitt ist eine (zusammenfassende) Aufzählung dessen, was LesMigraS will: »gesellschaftliche Veränderung«, eine »sensible« und »aktive Community«, »mehrdimensionale«, »langfristige« und »transformative« »Empowerment-Strategien«.

Die Anapher als rhetorisches Stilmittel nutzend, beginnen alle Sätze im letzten Abschnitt mit »Wir wollen«. Hier ist allerdings nicht das zuvor eingesetzte "gemeinsame Wir«, sondern das »LesMigraS-Wir« gemeint. Das äußert sich insofern, als Forderungen an die Community gestellt werden:»Wir wollen eine Community, die [...]«. Der CSD/die Community wird wieder als das Andere angerufen. Durch die Anapher wird eine besondere Betonung auf das gelegt, was LesMigraS will. LesMigraS konstituiert sich so als ein Wir, das nicht dadurch bestimmt ist, »was wir sind«, sondern »was wir wollen«. Durch die mantraartige Wiederholung der Wörter »Wir wollen« wird das Wir als durch sein Wollen bestimmt hergestellt. Im restlichen Satzteil wird jeweils das, was »wir wollen«, näher bestimmt. Alle diese Bestimmungen sind in den vorherigen Abschnitten bereits gefallen und werden hier noch einmal zusammengefasst. Das LesMigraS-Wir will eine »breite gesellschaftliche Veränderung« und eine Veränderung in der Community. Dabei wird die Community als potentielles Zuhause angerufen: »Wir wollen eine Community [...], in der sich Mehrfachzugehörige zu Hause fühlen können.« Ein (queer-feministisches) »Zuhause« kann mit Sarah Ahmed als »Zufluchtsort« verstanden werden. Die Forderung nach Teilhabe an der Community bekommt hier noch eine weitere Dimension. Es ist nicht nur eine Forderung nach Möglichkeiten der Repräsentation in einer Community oder die Forderung nach der "gemeinsamen Umsetzung von Handlungsstrategien« für eine »breite gesellschaftliche Veränderung«, es ist auch eine Forderung nach einem "Zuhause«, an dem mensch sich ausruhen kann, von all den Anstrengungen, die ein »feministisches Leben « mit sich bringt, das selbst jedoch auch Arbeit erfordert: »No wonder, if we are shattered, that we need places to go. [...] You have to work hard just for things to stay up. And I think that really affects the kind of work you do: you have less time to do things in the building when you are constantly doing building 
work. ${ }^{42}$ Im Fall von LesMigraS wird die Community explizit als ein Ort verstanden, an dem Menschen, die Gewalt und Diskriminierung erfahren (»if we are shattered«), Unterstützung suchen können (»we need places to go«). Indem LesMigraS Forderungen an den CSD stellt, wird ebendieser als Zuhause angerufen. Es ist der Versuch, an einem potentiellen Zuhause zu arbeiten, indem dieses Zuhause zum Ort der Verhandlungen und damit zum Ort der Öffnung gemacht wird.

\section{Die Community als Zuhause}

LesMigraS konstituiert sich selbst als eine Einrichtung, die sich über das bestimmt, was sie selbst will. Das, was LesMigraS will, wird gleichzeitig als Forderung an den CSD getragen. Damit ruft LesMigraS den CSD als potentielles "Zuhause « an, indem die Einrichtung die CSD-Gemeinschaft selbst zum Ort des Politischen macht. Während LesMigraS in diesem Jahr an keiner der Paraden teilnimmt, spricht die Einrichtung gleichzeitig über diese Nicht-Teilnahme, um im Sprechen darüber die Teilhabe am CSD einzufordern. Die Forderungen an den CSD äußern sich in Form von Gegensatzpaaren, die auf der einen Seite das beschreiben, was der CSD eigentlich sein sollte und was LesMigraS vom CSD fordert, und die auf der anderen Seite kritisieren, was der CSD aktuell ist und was den Grund darstellt, warum LesMigraS dieses Jahr an keiner CSD-Veranstaltung teilnimmt: Der CSD schließt Gruppen aus den »Entscheidungsprozessen um die Veranstaltungen« aus, äußert sich »entwicklungspolitisch zu anderen Ländern«, ist ein »eindimensionaler Feiertag« und nur »inklusiv«, wenn es »ins Programm passt«. Diesen Praktiken setzt LesMigraS positive Bestimmungen dessen entgegen, was den CSD zu einem »Zuhause« für LesMigraS machen würde: »[m]ehrdimensionales Empowerment«, "gesellschaftskritisches Engagement«, gemeinsames Kämpfen gegen »Mehrfachdiskriminierung«, sich »Kämpfen vor Ort anschließen«, »ganzjähriges Engagement «, »selbstkritische Auseinandersetzung «, »Austausch«, »unterschiedliche Bedürfnisse erfassen «, »diskriminierungssensible Umsetzung «, »Menschen mit Mehrfachzugehörigkeit in den Mittelpunkt der Arbeit stellen«, »aktiv einladen«. Die positive Bestimmung dessen, »was LesMigraS will«, changiert zwischen entleerten Begriffen, wie "gesellschaftliche Veränderung« oder "gesellschaftskritisch«, Begriffen, die an Diskurse der antirassistischen sozialen Arbeit anschließen - wie »Empowerment « oder »Sensibilität« - , und sehr konkreten Bestimmungen: »sich kämpfen vor Ort anschließen«, »Menschen mit Mehrfachzugehörigkeit in den Mittelpunkt der Arbeit stellen« oder »aktiv einladen«. Während die entleerten Begriffe eingesetzt werden, um die eigenen Forderungen, als solche, zu denen der CSD nicht Nein sagen kann, als totalisierende geltend zu machen, schließen insbesondere letztere an die eigene Arbeit von LesMigraS an. Die Einrichtung fordert einen Platz im CSD-»Zuhause«, indem das, was in der eigenen Arbeit zentral ist, als Wünsche und Forderungen an den CSD herangetragen wird. Über den performativen Widerspruch - als vom CSD Ausgeschlossene im Namen des CSD zu sprechen wird von LesMigraS eine CSD-Gemeinschaft gefordert, an der die Einrichtung teilhaben kann. 


\section{Welche Körper können auf der Pride erscheinen?}

Obwohl es sich bei den untersuchten Diskursfragmenten der Milchjugend und von LesMigraS um zwei verschiedene Textsorten handelt, die auf unterschiedliche Art und Weise funktionieren, unterschiedliche Intentionen haben und verschiedene Zielgruppen ansprechen, spielen beide eine Rolle bei der Herstellung des politischen Subjekts der jeweiligen Gruppe. In den Texten werden die politischen Subjekte der jeweiligen Projekte hergestellt, indem auf eine bestimmte Art und Weise das Verhältnis dieser Gemeinschaften zum CSD/zur Pride ausgelotet wird. Dieses Verhältnis wird anhand der Frage diskutiert, inwiefern der CSD/die Pride für diese Gruppen (k)ein Grund zum Jubeln ist. Während die Milchjugend die Pride als Erscheinungsraum des Politischen bejaht, sieht LesMigraS zumindest im Jahr 2014 in den CSD-Veranstaltungen keinen Grund zum Jubeln. Von beiden Gruppen wird der CSD als Ort des Ausschlusses verneint. Während die Milchjugend vor allem die durch die Kommerzialisierung produzierten Ausschlüsse ablehnt, lehnt LesMigraS den CSD vor allem als Ort rassistischer Ausschlüsse ab. Trotz der Verneinung eines durch Kommerzialisierung ausschließenden CSD, bejaht die Milchjugend die Pride als »Umzug für die Falschsexuellen«. LesMigraS hingegen sagt: Ja, wir wollen den CSD, aber nicht auf diese Art und Weise. Während die Milchjugend die Pride als Möglichkeitsraum/Ausgangspunkt des Politischen (ein Ort, an dem "gemeinsam gegen Heteronormativität angerannt« wird) bejaht und im eigenen Erscheinen gleichzeitig einfordert, arbeitet LesMigraS »on and against « ${ }^{43}$ den CSD, indem mit der NichtTeilnahme der CSD als das gefordert wird, was er eigentlich ist. Vereinfacht ausgedrückt: Die Milchjugend sagt, ja, wir erscheinen auf der Pride, um politische Transparente hochzuhalten, anstatt Bier zu trinken. LesMigraS sagt, nein, wir erscheinen nicht am CSD und nutzen die Nicht-Teilnahme, um einen anderen CSD zu fordern. Die Milchjugend sagt Ja zu den euphorischen Affekten, die die Pride auslöst, indem sie Bilder von Körpern auf der Straße aufruft, die das Politische und Kommerzielle der Pride miteinander vereinbaren können. LesMigraS hingegen macht einen performativen Widerspruch geltend, um das zu Beginn offengelegte Paradox zwischen dem, was der CSD eigentlich sein sollte, und dem, was er aktuell ist, aufzulösen. Es wird im Namen eines gemeinsamen Wir gesprochen, um genau dieses Wir einzufordern. Dabei wird eine gewisse Identität zwischen dem, was LesMigraS will und in der eigenen Arbeit macht, und dem, was der CSD eigentlich sein sollte, hergestellt. Auch die anderen Projekte, TransInterQueer e.V., das Jugendnetzwerk Lambda (BB) und die Türkis Rosa Lila Villa, konstituieren sich als bestimmte politische Subjekte, wenn sie auf eine spezifische Art und Weise das jeweils eigene Verhältnis zu den in ihren Städten stattfindenden CSD-, Pride- und Regenbogenveranstaltungen ausloten.

\subsubsection{Es gibt viele Arten, zur Pride Ja zu sagen}

Ähnlich wie LesMigraS nimmt auch die Berliner Einrichtung TransInterQueer e.V. (TrIQ) am alternativen Transgenialen CSD teil und bringt sich mit eigenen Redebeiträgen ein. In dem für die Feinanalyse ausgewählten Diskursfragment - einem Redebeitrag 
zum Transgenialen CSD (TCSD) 2013 - spricht TrIQ davon, wie eine »TransInterQueerTopia ${ }^{44}$ aussehen könnte. ${ }^{45}$ Ähnlich wie LesMigraS fordert auch TrIQ einen offeneren CSD, der solidarisch Trans* - und Inter*-Themen unterstützt. Die Forderungen von TrIQ werden ebenfalls aus einer spezifischen doppelten Bestimmung dessen, was der CSD eigentlich ist und sein sollte, begründet. Zum einen ist der CSD aufgrund seiner Bewegungsgeschichte ein möglicher Ort für Trans* - und Inter*-»Allies « ${ }^{46}$, denn die Geschichte des CSD ist eine über den "Schulterschluss von Drag Queens, Cross Dressern, Transsexuellen, Transgendern, Butches, Queers, Schwulen, Lesben, Femmes, Sexarbeiter*innen und Bi-Personen«. Zum anderen ist er aber auch ein Ort für Trans* - und Inter*-Themen, weil von ihm schon immer Kämpfe "gegen staatliche und medizinische Normierungen« ausgehen. Basierend auf dem bewegungsgeschichtlichen Hintergrund des CSD legt TrIQ fest, was der CSD eigentlich ist: Zum einen zeigen die »Stonewall Inn«-Proteste, dass der CSD immer schon ein Ort der Bündnisse mit Trans*-Personen und anderen war, $\mathrm{zu}$ anderem sind vom CSD schon immer Forderungen nach rechtlicher Anerkennung ausgegangen. So weit sagt TrIQ Ja zum CSD. Ähnlich wie LesMigraS lehnt aber auch TrIQ bestimmte Formen, die der CSD gegenwärtig angenommen hat, ab: einen CSD, an dem Trans* - und Inter* "Anhängsel eines schwul-lesbischen Aktivismus « sind, und einen CSD, an dem ein Kampf um Diskriminierung bedeutet, sich nur für die »bürgerlichen Rechte« von LGB-Menschen einzusetzen. Stattdessen schreibt TrIQ ein CSD-Ideal des solidarischen Bündnisses mit Trans* und Inter* im Kampf um deren rechtliche Anerkennung als »logisch « folgenden Schritt fest, denn LGB-Personen hätten sich - im Gegensatz zu Trans*-Personen - »mittlerweile« verschiedene Rechte erkämpft. Es sei, das suggeriert die Argumentation, jetzt an der Zeit, gemeinsam mit Trans*- und Inter*-Personen gegen medizinische Bevormundung und rechtliche Normierungen und Ungleichheiten vorzugehen. Neben dem Zeit-Argument wird auch das Argument angeführt, dass Menschen, die von Mehrfachdiskriminierung betroffen sind, härter getroffen seien: »Dabei trifft es jene, die Mehrfachdiskriminierung ausgesetzt sind, umso härter.« TrIQ bejaht bewusst den TCSD als einen Ort, von dem aus Gruppen im Namen ihrer Identitäten Anerkennung und Akzeptanz vom Staat fordern, um aus diesem Ort einen zukünftigen Ort zu machen, von dem aus gemeinsam gegen die medizinische und rechtliche Normierung von Trans* und Inter* vorgegangen wird. Sie bejahen den TCSD in einer spezifischen Lesart, die es ihnen möglich macht, auf einen zukünftigen TCSD zu verweisen, an dem Cis-Personen Trans*- und Inter* Themen unterstützen, weil es an der Zeit ist, diejenigen zu unterstützen, die es umso härter trifft.

44 Das ist das Motto des TCSD 2013.

45 TransInterQueer e.V.: Redebeitrag zum Transgenialen CSD 2013. TransInterQueer-Topia 2013, http s://www.transinterqueer.org/download/medien/pdf/TCSD_2013_Statement.pdf vom 20.11.2020.

46 Als Allies gelten zumeist Personen, die selbst nicht trans* oder inter* sind, die aber deren Themen und Kämpfe unterstützen. Dabei gibt es bestimmte Regeln, die Trans*-Allies einhalten sollten (z.B. nach dem bevorzugten Pronomen fragen, keine intimen, unzulässigen Fragen stellen, Trans* Personen zuhören, nicht vom Aussehen auf das Geschlecht schließen oder nicht ohne Erlaubnis outen). Vgl. René_Hornstein: Trans*verbündetenschaft. Was wünschen sich Trans*personen von Menschen in ihrer Umgebung an unterstützendem Verhalten? Diplomarbeit, Osnabrück 2017. 
Ebenfalls in Form eines Redebeitrags am Berliner CSD (nicht am Transgenialen CSD) positioniert sich das Jugendnetzwerk Lambda BB zum CSD. ${ }^{47}$ Anlass der Rede ist die Entgegennahme des »Soul of Stonewall Award«, der im Jahr 2015 an Lambda BB vergeben wurde. Entsprechend wird die Rede vom Landesvorstand des Jugendnetzwerks Lambdas BB gehalten. Nachdem in der Dankesrede zunächst davon gesprochen wird, wer das Jugendnetzwerk Lambda BB sei (engagiertes Team, ehrenamtliche Helfer*innen, Interessenvertretung für LGBTIQ*-Jugendliche, einziger queerer Jugendverband) und was Lambda BB mache (Vertretung in Politik und Öffentlichkeit, öffentliche Demonstrationen, Bildungsarbeit, Schutzräume schaffen), wird das besondere Verhältnis zum CSD ausgelotet. Lambda BB wird durch den Award für das besondere »Engagement « ${ }^{48}$ für die Community ausgezeichnet und nimmt diese Anrufung an. Damit positioniert sich das Netzwerk als »besonderer und wichtiger Teil« der (CSD-)Community. Die eigene Besonderheit und Wichtigkeit wird in der Dankesrede besonders hervorgehoben, wenn Lambda BB schreibt, dass sie »der einzige Jugendverband von und für lesbische, schwule, bisexuelle, trans*, inter* und queere Jugendliche in Berlin Brandenburg sind «. Im quantitativ größten Teil der Rede nutzt Lambda BB die »Bühne« des CSD, um die eigene Arbeit und die Einrichtung vorzustellen. Lambda $B B$ positioniert sich aber auch zum CSD als Ort, der durch die Annahme des Preises affirmiert wird. Lambda BB nimmt den Preis von einem CSD an, der »an das Erbe und den Geist« der »Stonewall Inn«Proteste im Jahr 1969 erinnere: »Kämpfe von Trans*, Drag-Queens, LSBTIQ* of Color [...] gegen alltägliche Gewalt«. Den Award im Namen dieser Kämpfe anzunehmen, verpflichte dazu, Mehrfachdiskriminierung und Ausschlüsse zu benennen:

»Nicht nur, aber auch durch die Entgegennahme des Publikumspreises der >Soul of Stonewall Awards` des CSD e.V. fühlen wir uns verpflichtet, alltägliche Gewalt, Sexismus, Rassismus, Fremdenfeindlichkeit, Homo-Bi und Transphobie, Diskriminierung aufgrund des Alters, der Religionszugehörigkeit, aufgrund von Behinderung oder klassische Diskriminierung sowie Mehrfachdiskriminierungen zu thematisieren und entgegenzuwirken.«

Der Award und damit die Anrufung als besonderer Teil der CSD-Community wird angenommen, indem diese Anrufung bewusst im »Erbe und Geist « dessen, worum es bei den »Stonewall Inn«-Protesten wirklich ging, (miss-)verstanden wird. Lambda BB versteht die Anrufung absichtlich »falsch « und verweist so auf die Diskrepanz zwischen der missverstandenen und ausgerufenen Anrufung. Auch hier wird - ähnlich wie bei LesMigraS und TrIQ - im Namen eines eigentlichen CSD, der durch seine Geschichte bestimmt ist, Kritik am aktuellen CSD geübt. Lambda BB kritisiert, dass nur »mehrheitsweiße Organisationen für den Publikumspreis nominiert werden«. Das, was der CSD eigentlich ist, wird durch eine eindeutige, unmissverständliche Erzählung dessen, was »Erbe und Geist« der »Stonewall Inn«-Proteste ist, festgeschrieben: ein Kampf gegen »rassistische, Trans*diskriminierende und klassistische Polizei- und Staatsgewalt«.

47 Jugendnetzwerk Lambda:BB: Stellungnahme des Jugendnetzwerks Lambda Berlin-Brandenburg auf dem Berliner Christopher Street Day am 27. Juni 2015, https://www.lambda-bb.de/wp-content /uploads/2012/12/Stellungnahme-Lambda-zu-CSD_030820105-.pdf vom 20.11.2020. 
Gleichzeitig nutzt das Jugendnetzwerk Lambda BB die durch diese Geschichtserzählung eröffnete Möglichkeit - nämlich die Anrufung durch den CSD »missverstanden« anzunehmen -, um die eigene Arbeit und das, was Lambda BB ist, sichtbar zu machen. Lambda BB bejaht den CSD als Erbe der »Stonewall Inn«-Proteste, also als Kampf gegen Gewalt und Mehrfachdiskriminierung. Lambda BB bejaht aber gleichzeitig auch den CSD als Ort, der dem Netzwerk eine Bühne zur Selbstdarstellung gibt.

In ihrem kurzen Bericht zur Regenbogen-Parade 2014 in den »Lambda-Nachrichten« sagt auch die Türkis Rosa Lila Villa auf eine bestimmte Art und Weise Ja zur Regenbogenparade, wenn beschrieben wird, wie die Einrichtung an der Parade teilnimmt. ${ }^{49}$ Die Teilnahme an der Regenbogenparade ist bestimmt durch Kooperationen und Solidarität mit anderen, vor allem antirassistischen und migrantischen Gruppen: »Die Rosa Lila Villa organisierte mit etlichen anderen Gruppen einen queer-feministischen, migrantischen und antirassistischen Truck, der ein großer Erfolg war«. Die Villa sagt Ja zur Regenbogenparade und stellt gleichzeitig durch die Teilhabe an der Regenbogenparade alternative Räume her. Das Hausprojekt berichtet von Solidarisierungen mit migrantischen Gruppen beziehungsweise davon, gemeinsam als »antirassistischer«, »migrantischer«, »queer-feministischer Truck« auf der Regenbogenparade einen Ort für jene Gruppen zu schaffen, die sich durch die bestehenden Räume auf der Regenbogenparade nicht repräsentiert sehen. Sie »bejahen« die Regenbogenparade als Ort des Ausschlusses, der gleichzeitig die Möglichkeit bietet, Räume für die von der Regenbogenparade Ausgeschlossenen zu schaffen. Ähnlich wie die Milchjugend an der Pride, nimmt die Türkis Rosa Lila Villa an der Regenbogenparade teil, um diese durch die eigene Teilhabe als den Ort herzustellen, der die Parade sein soll. Anders als die Milchjugend, die über die körperlichen Fähigkeiten verfügt, die Pride - trotz Kommerzialisierung - zum Erscheinungsort des Politischen zu machen, nimmt die Türkis Rosa Lila Villa (im Folgenden kurz: Villa) die Regenbogenparade als einen Möglichkeitsraum, der im Erscheinen selbst »offener« gestaltet werden kann, an. Die Villa macht so - ähnlich wie LesMigraS - die Anordnung der Regenbogenparade selbst zum Ort des Politischen. Anders als LesMigraS, das den CSD durch die Nicht-Teilnahme zum Kampfschauplatz macht, stellt die Villa durch ihre Teilnahme die Regenbogenparade als den Raum her, den sie fordert: einen solidarischen, antirassistischen, offenen Raum. ${ }^{50}$ Die Villa spricht in ihrem Bericht davon, wie in und durch konkrete eigene Praktiken auf der Regenbogenparade alternative Räume hergestellt werden, in denen die Villa auf der Regenbogenparade schließlich einen Grund zum Jubeln hat.

Der in der Zeitschrift »Out!« des Jugendnetzwerks Lambda ${ }^{51}$ veröffentlichte Artikel »CSD - Politik oder Konsum ${ }^{52}$ weist Ähnlichkeiten im Aufbau der Argumentation mit

49 Türkis Rosa Lila Villa: »Willkommen im Korridor! Parade 2014«, in: Lambda-Nachrichten vom JuliAugust 2014, https://lambdanachrichten.at/ln143.pdf vom 20.11.2020, S. 16.

50 Auch LesMigraS hat regelmäßig an TCSDs und KCSDs teilgenommen und alternative Räume an diesen Umzügen geschaffen.

51 »Out! « ist die Zeitschrift des Bundesverbandes des Jugendnetzwerks Lambda, der dem Berliner Jugendverband sehr nahesteht, aber nicht identisch mit diesem ist. Der Artikel wurde zusätzlich zur Stellungnahme des Berliner Jugendverbandes gewählt, weil die Argumentation eine andere ist. Jugendnetzwerk Lambda: »CSD - Politik oder Konsum«, in: Konsum, Out! 2012. 
dem bereits vorgestellten Artikel der Milchjugend auf. Das liegt wahrscheinlich auch daran, dass es sich - im Gegensatz zu den Stellungnahmen und Redebeiträgen, die sowohl an die eigene Community als auch an die Öffentlichkeit gerichtet sind - um einen Kommentar in einer projekteigenen Zeitschrift handelt, die hauptsächlich die eigene Community anspricht. Ähnlich wie der Artikel der Milchjugend beginnt auch der »Out!«-Text die Auslotung des Verhältnisses der eigenen Gemeinschaft zum CSD mit einer Darstellung der Ausschlüsse der Pride/des CSD - insbesondere einer Kritik an der Kommerzialisierung -, die in einem (vermeintlichen) Gegensatz zum Politischen des CSD steht (»Politik oder Konsum?«). Dabei dreht sich auch in der »Out!« die Auslotung des Verhältnisses zum CSD maßgeblich um die Frage, ob »ein kommerzialisierter CSD noch Sinn macht«. Im Unterschied zur Milchjugend werden die zwei sich widersprechenden Aspekte des CSD - das Politische und das Kommerzielle - aber nicht auf einer primär affektiven Ebene evident gemacht. Vielmehr wird - ähnlich wie es bisher in fast allen Argumentationen der Fall war - die Geschichte der »Stonewall Inn«-Proteste als für das Politische des CSD stehende postuliert und die dem entgegenstehende Kommerzialisierung und die Ausschlüsse des CSD werden in Bezug auf Kritiken aus der Community benannt. Hier wird die immer »lauter werdende Kritik an der Kommerzialisierung «, aber auch die Kritik durch den Transgenialen CSD und Judith Butler vor allem am antimuslimischen Rassismus des Berliner CSD - hervorgehoben. Ähnlich wie die Milchjugend positioniert sich auch das Jugendnetzwerk Lambda zu den Kritiken aus der Community am CSD. Während die Milchjugend allerdings für eine Gleichzeitigkeit von »Politik und Konsum« plädiert, wird im Diskursfragment der »Out!« die Entscheidung für oder gegen den CSD zu einer individuellen Entscheidung gemacht: »Es liegt in der Verantwortung einer_eines jeden Einzelnen, wohin sie_er geht, wofür sie_er jubelt«. Es wird kein Wir hergestellt, das die Pride trotz Kommerzialisierung bejaht (Milchjugend), kein Wir, das den CSD in einer bestimmten Lesart und aufs Zukünftige verweisend bejaht (TrIQ), kein Wir, das alternative Räume auf der Regenbogenparade herstellt (Türkis Rosa Lila Villa), und auch kein Wir, das das Wir des CSD selbst zum primären Verhandlungsort macht (LesMigraS). Es wird ein Wir hergestellt, das keinen Konsens in Bezug auf das Verhältnis zum CSD braucht, ein Wir, das aus vereinzelten Individuen besteht, die jeweils für sich eine Entscheidung treffen. Der einzige Konsens, der hergestellt wird, ist der, dass die Einzelnen »bewusst « entscheiden sollen, wie sie sich zum CSD verhalten: "Wir können uns entscheiden, uns und die relative Freiheit zu feiern, uns mit Stolz zu zeigen und riesige Partys zu bevölkern. Und wir können den Sexismus und Rassismus auf den Flyern der CSD-Partys beachten und kritisieren [...]. Wichtig ist nur, dass jeweils das Bewusstsein dafür da ist, was wir warum tun [...].« Egal ob die Entscheidung für oder gegen den CSD ausfällt, wird doch das Politische als die handlungsleitende Maxime festgeschrieben, die wiederum bewegungsgeschichtlich legitimiert wird: "gerade auch in Gedenken an die Stonewall riots«. Auch hier wird das Politische des CSD als gemeinsamer Nenner bejaht, es wird aber nicht festgeschrieben, was das Politische des CSD ist. Der CSD wird weder als ein Ort noch als kein Ort zum Jubeln eines kollektiven Wir hergestellt, sondern die Antwort auf die Frage, ob der CSD noch Sinn mache, wird zu einer individuellen (bewussten und reflektierten) Entscheidung. Die Konsequenz aus der Widersprüchlichkeit des CSD - er ist gleichzeitig ein und kein Ort zum Jubeln - ist die, dass kein gemeinsames Verhältnis zum CSD 
ausgelotet werden kann. Es gibt kein gemeinsames Ja zum »Transparente halten statt Bier trinken«, keine gemeinsame Forderung nach Einschluss, keine Forderungen nach gemeinsamen Bündnispolitiken gegen staatliche Normierung und kein gemeinsames Herstellen von alternativen Räumen auf dem CSD, sondern Einzelne, die entscheiden, ob sie Bier trinken, Transparente halten, die Einladungspolitik kritisieren, Einzelne, die entscheiden, ob sie auf den Transgenialen CSD, den Berliner CSD oder auf keinen CSD gehen.

Auch wenn oberflächlich betrachtet alle fünf Projekte fast immer an den CSD-PrideRegenbogenparaden teilnehmen und ihnen allen gemeinsam ist, dass sie eine politische Pride wollen, loten die Gruppen im Sprechen über den CSD das eigene Verhältnis zu ebendiesen doch auf eine jeweils spezifische Art und Weise aus. Gemeinsam ist allen Projekten, dass sie sich mit ihrer Teilnahme oder Nicht-Teilnahme zum CSD positionieren. Dabei versuchen die Gruppen teilzuhaben an den Prozessen, in denen hergestellt wird, was der CSD ist. Während manche an diesen Prozessen teilhaben, indem sie auf eine bestimmte Art und Weise am CSD erscheinen, kritisieren andere im Namen des eigentlichen CSD, den sie gleichzeitig einfordern, den aktuellen CSD. Die Milchjugend bejaht die Pride trotz Kommerzialisierung als Erscheinungsort des Politischen und sagt damit nach einem langen Reflexionsprozess Ja zur anfänglichen Euphorie. LesMigraS macht die ein- und ausschließenden Praktiken des CSD selbst zum Ort des Politischen, indem Einschluss in ebendiese gefordert wird. TrIQ verweist auf den CSD als zukünftigen Ort für Trans* - und Inter* -Personen, indem der CSD in einer bestimmten Lesart an seine Geschichte der Bündnispolitiken gekoppelt wird und er, als an den Staat Anspruch erhebend, bejaht wird. Das Jugendnetzwerk Lambda BB bejaht in der Dankesrede den CSD im »Geist und Erbe« der »Stonewall Inn«-Proteste, was dazu verpflichtet, auf Ausschlüsse in der Community zu verweisen. Dieses Ja eröffnet aber gleichzeitig auch die Möglichkeit, das eigene Projekt auf der »Bühne« des CSD sichtbar zu machen. Die Villa bejaht die Regenbogenparade als einen Ort, an dem alternative Räume für die durch die Paraden nicht Repräsentierten hergestellt werden können. Und in der »Out!« des Jugendnetzwerks Lambda wird die Frage, ob der CSD als politischer Ort bejaht werden könne, zu einer Frage der individuellen Entscheidung und Verantwortung. Welche Rolle spielen nun aber Identitäten oder Identitätspolitiken - vor dem Hintergrund, dass »queer « aus einer Kritik an eindeutigen Identitäten und Identitätspolitiken heraus entstanden ist - in diesen verschiedenen Arten, das jeweilige kollektive Wir in Bezug auf die Pride zu konstituieren? Wenn Identität ein auf Schließung abzielender Prozess ist und »queer« demgegenüber als offenes Projekt verstanden wird, welchen Grad der Schließungen nehmen die jeweiligen Aushandlungen des eigenen Verhältnisses zum CSD an?

\subsubsection{Identität bezeichnet nicht, was wir sind}

Welche Rollen spielen Identitäten für die Art und Weise, wie die Milchjugend Ja zur anfänglichen »Euphorie« und damit zur Pride als »Erscheinungsort« des Politischen sagt? Auf der Ebene konkret verwendeter Identitäts-Kategorien findet sich im ganzen Diskursfragment nur die Bezeichnung »falschsexuell« als Selbstbezeichnung, die 
als Identitätsbezeichnung gelten könnte. Die Milchjugend selbst versteht »falschsexuell« allerdings als deutsche Übersetzung von "queer« und damit eben als eine nichtidentitätspolitische, subversive Bezeichnung. ${ }^{53}$ Die Milchjugend stellt im Verhältnis zur Pride ein kollektives Wir her, für das eine Gleichzeitigkeit von Politik und Kommerzialisierung auf der Pride möglich ist. Die Pride als Erscheinungsort des Politischen ermöglicht es »Körpern« die Straße einzunehmen, "gegen Heteronormativität anzurennen« und »Transparente zu halten«. Die Milchjugend schreibt in der Auslotung des Verhältnisses zur Pride eine Idee des Politischen fest, nach der Körper auf der Straße in Erscheinung treten, den öffentlichen Raum einnehmen, sichtbar werden, und Forderungen stellen. Es werden Körperpolitiken und keine Politiken, die im Namen einer Identität sprechen und Forderungen stellen, bejaht. Es wird auch nicht davon gesprochen, Rechte oder Anerkennung für bestimmte um Identität angeordnete Gruppen einzufordern, sondern gegen »Heteronormativität anzurennen«. Das heißt, im Sprechen über ihr Verhältnis zur Pride werden zunächst einmal keine offensichtlich identitätspolitischen Rhetoriken angestimmt. Das Verhältnis zur Pride wird von der Milchjugend nicht über eine Bestimmung dessen, »was sie sind«, sondern über affektive Anrufungen und Körperbilder eingefordert.

Wird die Frage nach Bestimmung des Verhältnisses zu Identitätspolitiken jedoch in Bezug auf den Grad der Schließung gestellt, kommt die Frage auf, welche Körper innerhalb des kommerzialisierten Raums der Pride in Erscheinung treten können und welche nicht. Nicht nur Identitäten, auch die affektiven Anrufungen und Körperbilder verweisen auf einen bestimmten Platz, von dem aus die Pride trotz Kommerzialisierung ein Grund zum Jubeln darstellt. Was ist aber mit den Körpern, die diesen Platz nicht einnehmen können? Die, die nicht durch das euphorisierende Gefühl »Teil« einer Gemeinschaft zu sein angerufen werden können oder wollen, weil sich vielleicht der Schmerz und die Wut (historisch) in ihre Körper eingeschrieben hat ${ }^{54}$ Was ist mit den Körpern, die ohne »Unterstützungsmöglichkeiten ${ }^{55}$ nicht dazu in der Lage sind, »Transparente zu halten und Werbeflyer in die Tasche« zu stecken? Bejaht die Milchjugend die Pride als einen Ort, der eine bestimmte körperliche Konstitutionen - Transpa-

53 Milchjugend: Hauptgang.

54 Diese Gedanken nehmen Bezug auf einen Artikel von Ann Cvetkovich, in dem es auch um das Politisierungspotential von Schwarzer Wut und Depression geht, die sich zum Teil historisch in Schwarze Körper eingeschrieben haben. Umgekehrt bleiben Schwarzen Körpern, Cvetkovich zufolge, »privilegierte«, »feel good«-Politiken allerdings häufig verschlossen. Vgl. Cvetkovich, Ann: »Depression ist etwas Alltägliches: Öffentliche Gefühle und Saidiya Hartmans `Lose Your Mother «, in: Angelika Baier/Christa Binswanger/Jana Häberlein et al. (Hg.), Affekt und Ceschlecht. Eine einführende Anthologie, Wien: Zaglossus Verlag 2014, S. 57-86.

55 Auch hier wird implizit Bezug auf das, was Judith Butler mit Blick auf Perspektiven aus den Disability Studies »Infrastrukturen«nennt, genommen. Unter Infrastrukturen versteht Butler das »komplexe Geflecht aus Umwelt, Sozialbeziehungen, Unterstützungs- und Versorgungsnetzwerken«, die nicht vom Erscheinungsraum des Politischen zu trennen sind. Die Straße muss auf eine bestimmte Art und Weise beschaffen sein, damit bestimmte Menschen - beispielsweise mit einer körperlichen Behinderung - erscheinen können. Es kann aber auch um fehlende Infrastrukturen in Bezug auf jene gehen, für die ein Erscheinen auf der Straße beispielsweise aufgrund eines ungesicherten Aufenthaltsstatus prekär ist. J. Butler: Anmerkungen zu einer performativen Theorie der Versammlung, S. 175. 
rente halten und Werbeflyer einstecken - und affektive Stimmungen voraussetzt und die damit jene ausschließt, die diese Voraussetzungen nicht erfüllen? Oder fordert die Milchjugend dadurch, dass sie auf einer Straße erscheint und die Pride im Erscheinen als einen Erscheinungsraum des Politischen einsetzt, eine Pride, auf der alle »Transparente halten« können?

Andere, jenseits der Kommerzialisierung stattfindende Ausschlüsse, wie beispielsweise rassistische- oder transphobe Ausschlüsse bleiben in der Diskussion um die Pride gänzlich unerwähnt. ${ }^{56}$ Gleichzeitig ist die Milchjugend eine Gruppe, die die finanziellen Möglichkeiten hat, an der kommerzialisierten Pride teilzunehmen. Die Jugendpride ist eine Welt, die die Milchjugend Geld kostet. Die Einrichtung kann Ja zu Gleichzeitigkeit von Politik und Konsum sagen, weil sie es sich leisten kann. Gleichzeitig überlässt die Milchjugend, als eine Gruppe, die es sich leisten kann, den Platz (die Pride) nicht einfach denjenigen, die ihn einzig als kommerziellen Ort bejahen. In und durch ein Ja zu einer politischen Pride setzt sich die Milchjugend für den CSD als Erscheinungsort des Politischen und gegen einen kommerzialisierten CSD (Transparente hochhalten statt Bier trinken) ein. Die Teilnahme an diesem Kampf ist allerdings nur für die Menschen möglich, die auf den Zug der Pride-Euphorie aufspringen können und deren Körper die Fähigkeit besitzen, gleichzeitig Transparente und Werbeflyer zu tragen.

LesMigraS verwendet im Vergleich zur Milchjugend sehr wohl (Mehrfach-)Identitätsbezeichnungen, wie beispielsweise die Bezeichnungen "geflüchtete LSBTIQ« oder »LSBTIQ of Color «, wenn sie den Einschluss ebenjener Gruppen in den CSD fordern. Sie selbst verstehen solche Bezeichnungen als »Identitäts- oder ZugehörigkeitsKategorien«, die beschreiben, dass Personen bestimmten (oft mehreren) Gruppen angehören. Diese Zugehörigkeits-Kategorien ordnen nach dem Verständnis von LesMigraS die (machtvolle, ungleiche) Verteilung der Teilhabe an der Gesellschaft. ${ }^{57}$ Insofern sind es mit Judith Butler gesprochen Kategorien, die als »kulturell beschränktes Ordnungs- und Hierarchieprinzip« verstanden werden. ${ }^{58}$ LesMigraS spricht weder im Namen von »single-axis«-Kategorien, ${ }^{59}$ noch spricht LesMigraS von IdentitätsKategorien, die bezeichnen »wer sie sind «. ${ }^{60}$ Zugehörigkeits-Kategorien in dieser Weise zu verstehen und zu fassen, ist wohl auch eine Antwort auf die vielfältigen Kritiken an Identitätspolitiken. Auf der einen Seite findet hier eine Politik statt, die sich eindeutig von Identitätspolitiken als »single-axis-Politiken« abgrenzt und IdentitätsKategorien eben nicht als Beschreibung dessen versteht, was das kollektive Wir ist. Auf der anderen Seite werden vermeintliche Mehrfachidentitäts-Kategorien eingesetzt,

56 Die Milchjugend spricht in der Zeitschrift generell über andere Ausschlüsse, beispielsweise aufgrund von Rassismus oder Ableismus. Im Kontext der Pride spielt dieses Thema 2012 bis 2016 jedoch kaum eine Rolle.

57 LesMigraS: Was ist Mehrfachzugehörigkeit und Mehrfachdiskriminierung? 2012, https://lesmigras .de/tl_files/lesmigras/Tapesh/Texte/2015_MFD.pdf vom 20.11.2020.

58 J. Butler: Das Unbehagen der Geschlechter, S. 48.

59 K. Crenshaw: Demarginalizing the Intersection of Race and Sex: A Black Feminist Critique of Antidiscrimination Doctrine, Feminist Theory and Antiracist Politics, S. 140. 
von denen sogar gefordert wird, dass sie in den Mittelpunkt der Politik gestellt werden. Das, was sich verschoben hat ist - neben der Verwendung von Mehrfach-, statt Einfachidentitäts-Kategorien - die Bedeutung, die diesen Kategorien zukommt. Sie gelten oft nicht einfach nur als Identitäts-Kategorien, sondern als Kategorien, durch die ungleiche Verteilungen und Machtverhältnisse angeordnet werden. Entsprechend wird dem Einsatz dieser Kategorien nicht die Wirkung zugeschrieben, identitätspolitische Schließungen $\mathrm{zu}$ affirmieren, sondern Machtverhältnisse anders/neu anzuordnen. Allerdings sind es wieder Kategorien und Gruppen, die selbst von Machtverhältnissen durchzogen sind, die zum Einsatzort des Kampfes nach Einschluss und alternativer Verteilungen gemacht werden.

Ein weiterer Aspekt der Mehrfachidentitätsbezeichnung ist die Verwendung des Akronyms LSBTIQ. Das Kürzel LSBTIQ stellt eine, in queeren Kontexten gebräuchliche und häufig verwendete Art dar, um die vielfältigen Lebens- und Seinsweisen in der queeren Community zu repräsentieren. ${ }^{61}$ Es ist eine in der queeren Gemeinschaft geteilte Symbolik, die eine eigene Geschichte hat. Viele dieser Zugehörigkeiten, die in den Buchstaben-Kürzeln repräsentiert werden, mussten und müssen sich bis heute einen Platz in den, zu Beginn zumeist schwulen zum Teil auch lesbischen Repräsentationspolitiken erkämpfen. Die Kämpfe darum, wer in und durch die Abkürzungen wann und wie repräsentiert wird, finden nach wie vor statt und sind konstitutiv offen. ${ }^{62}$ Es wird aber häufig auch kritisiert, dass manche Lebens- und Seinsweisen $\mathrm{zu}$ Unrecht repräsentiert werden. ${ }^{63}$ Zumeist gilt die Regel: so vielfältig wie möglich, aber nicht vereinnahmend. Einen Buchstaben nicht zu vereinnahmen, heißt zumeist auch, ihn »authentisch « zu repräsentieren. Das setzt aber umgekehrt voraus, dass eine »authentische« Repräsentation möglich ist. ${ }^{64}$ Das Akronym steht aber auch in der Kritik, Identitäts-Kategorien zu vervielfältigen und aufzuzählen. Durch Aneinanderreihung und Vervielfältigung von Identitäts-Kategorien würden insofern Schließungen produziert, als Identitäten affirmiert werden. Es würden in und durch die Vervielfältigung aber auch neoliberale Bedürfnisse nach möglichst unterschiedlichen und einzigartigen Konsument"innen und einem unternehmerischen Selbst befriedigt. Die Aneinanderreihungen stellen also einen mehr oder weniger offenen Kampfschauplatz um Repräsentation dar. Wie und warum werden nun aber diese Zugehörigkeits-Kategorien in der Argumentation von LesMigraS verwendet? Sind sie notwendig? Und wenn ja, warum?

LesMigraS begründet die Forderung an den CSD, Menschen mit Mehrfachzugehörigkeiten in den Mittelpunkt zu stellen, nicht. Die beiden Male, in denen diese Forderung gestellt wird, geschieht dies allerdings in Abgrenzung zu den diese Gruppen ausschließenden Praktiken des CSD (z.B. entwicklungspolitische Äußerungen). Daher kann diese Forderung im Namen von »Mehrfachzugehörigkeits-Kategorien« als eine

61 Alle Projekte verwenden diese Akronyme, wenn auch mehr oder weniger häufig und auf unterschiedliche Art und Weise.

62 Mittlerweile werden z.B. häufig auch Menschen aus dem asexuellen Spektrum mit der Abkürzung A inkludiert.

63 Ähnlich kritisiert LesMigraS, dass der CSD den Anspruch erhebt alle zu repräsentieren, ohne sie wirklich zu repräsentieren. 
Forderung nach »Öffnung« der ausschließenden CSD-Gemeinschaft verstanden werden. Gleichzeitig scheinen diese Forderungen, gerade aufgrund der ausschließenden Praktiken des CSD, notwendig zu sein. Im Gegensatz zur Milchjugend verfügt LesMigraS nicht über die notwendigen Möglichkeiten (körperlichen Fähigkeiten), trotz der ausschließenden Praktiken des CSD auf der Straße zu erscheinen. Es scheint, ähnlich wie Petra Rostock es am Beispiel von »FeMigra« beschreibt, dass gerade Mehrfachzugehörige zum Teil derart auf ihre Zugehörigkeiten zurückgeworfen werden, dass ihr Einsatz notwendig ist. ${ }^{65}$ Die Kategorien sind in der realen Welt, mit Crenshaws Worten, derart wirkmächtig, dass sie politisch aufgegriffen werden müssen. ${ }^{66}$ Offen bleibt, welche Menschen mit welchen Mehrfachzugehörigkeiten in den Mittelpunkt der CSDArbeit gestellt werden sollen und was das für die Arbeit bedeutet, wenn, mit Spivak gesprochen, nicht von einem »homogenen subalternen Subjekt« gesprochen werden kann. ${ }^{67}$ Die Art und Weise, wie die Zugehörigkeits- oder Identitäts-Kategorien verwendet werden, führt bereits mitten in die Diskussion um die Frage nach dem Grad der Schließung, auf den die Konstitution von LesMigraS als kollektives Wir, das durch ein bestimmtes Verhältnis zum CSD ausgelotet wird, (unbewusst) abzielt.

Das Verhältnis zwischen LesMigraS und dem CSD wird vor allem jenseits der Verwendung von Zugehörigkeits-Kategorien artikuliert. Anrufungstheoretisch verweigert LesMigraS als Gruppe die Unterwerfung unter die Anrufungen des CSD und damit die Unterwerfung unter eine sich durch ausschließende Praktiken konstituierende CSDGemeinschaft. Die Einrichtung weigert sich, eine "Identität« zwischen sich und dem CSD herzustellen, indem sie sich der "geschlossenen « Anrufung nicht unterwirft. Stattdessen macht LesMigraS die Schließungen der Anrufungen, die die Einrichtung auf einen bestimmten Platz verweisen möchte, selbst zum Thema. Anstatt sich den einfachen Anrufungen durch den CSD zu unterwerfen, fordert LesMigraS einen Einschluss dessen, was durch die Praktiken des CSD nicht repräsentiert wird, und macht so den CSD selbst zum Ort der Verhandlung und Öffnung. In den Forderungen nach einer Öffnung der CSD-Gemeinschaft wird allerdings umgekehrt eine gewisse Identität zwischen dem, was LesMigraS politisch »will« und »tut«, und dem, was für den CSD gefordert wird, hergestellt. Verweigert LesMigraS auf der einen Seite eine identische Beziehung mit dem CSD, indem sich die Einrichtung von ihm nicht auf einen Platz verweisen lässt, so fordert LesMigraS auf der anderen Seite einen CSD, dessen politische Praktiken (beinahe) identisch mit denen von LesMigraS sind (mehrdimensionales Empowerment, Sensibilität, Offenheit, diskriminierungssensibel). Dabei stellt sich LesMigraS gleichzeitig - in Abgrenzung zum CSD - als eine bereits offenere Gemeinschaft her. Es wäre zu diskutieren, inwiefern dieser Kampf um eine Öffnung des CSD selbst ein offener ist. Ist die durch die Forderung hergestellte identische Beziehung (zwischen dem, was LesMigraS »will«, »tut« und »ist«, und dem, was der CSD »sein soll«) eine notwendige politische Strategie oder handelt es sich um eine umgekehrte Form der Schließung - die andere

65 P. Rostock: Jenseits von >Identität«? Zu den Un/Möglichkeiten nicht-identitärer Strategien politischen Handelns, S. 193.

66 K. Crenshaw: Mapping the Margins: Intersectionality, Identity Politics, and Violence against Women of Color, S. 1296.

67 G. C. Spivak: Can the Subaltern Speak?, S. 284. 
Seite der Medaille? Welche Schließungen werden produziert, wenn LesMigraS sich in Abgrenzung zu den Forderungen nach einem offeneren CSD selbst als die offene Gemeinschaft par excellence konstituiert?

Auch das sich selbst als »mehrheitsweiße« Organisation bezeichnende Jugendnetzwerk Lambda BB fordert im »Geiste Stonewalls« einen Einschluss von »queeren, nichtmehrheitsweißen Vereinen«. Der Einsatz für den Einschluss von Menschen mit Mehrfachdiskriminierungserfahrungen muss - so der Landesvorstand in der Dankesrede - »insbesondere auch für Menschen mit Privilegien wie mich, die sich beispielsweise als schwul, cis-männlich und weiß identifizieren«, gelten. Hier wird eine Beziehung zwischen der Identifikation mit (»privilegierten«) Identitäten und dem Einsatz und Ort des Politischen hergestellt. »Was wir sind«, bestimmt, »was wir fordern/tun«. Auf der separaten Homepage des peer-to-peer-Projektes »queer@school ${ }^{68}$ des Jugendnetzwerks Lambda $B B$ wird näher bestimmt, wie das Verhältnis von Identitäten und Privilegien verstanden wird: »Alle Menschen haben immer mehrere Identitäten (Mehrfachzugehörigkeit) und erleben daher in Bezug auf ihre Identitäten mehrere Diskriminierungen und Privilegierungen. $\aleph^{69}$ Diskriminierung und Privilegierung werden wiederum als etwas verstanden, das auf »individueller«, »institutioneller « und »struktureller « Ebene stattfindet. Über die beiden Begriffe »Privilegien« und »Diskriminierung« werden die Identifikations-Kategorien auch mit »Machtverhältnissen« verknüpft: »Machtverhältnisse führen dazu, dass bestimmte Personengruppen Vorteile gegenüber anderen Personengruppen haben. Bevorteilung (Privilegierung) geschieht auf Kosten von Benachteiligung (Diskriminierung) bestimmter Gruppen. " ${ }^{70}$ Machtverhältnisse schaffen, dieser Argumentation folgend, einerseits die, die profitieren (»die Privilegierten«), und andererseits die, auf deren Kosten die Privilegierung geht (»die Diskriminierten «). ${ }^{71}$ Identifikations-Kategorien werden hier als Ordnungsschemata verstanden, die über Privilegierungen und De-Privilegierungen in der Gesellschaft bestimmen. Lambda BB positioniert sich innerhalb eines bestimmten Critical-Whiteness-Diskurses, der im deutschsprachigen Raum unterschiedlich diskutiert und in die Praxis umgesetzt wird.

Exkurs Critical Whiteness (CW): Critical Whiteness wird als ein wichtiger Bestandteil antirassistischer Theorie und Praxis im deutschsprachigen Raum verstanden. Je nach Umsetzung und Verständnis gehen mit CW allerdings auch Schwierigkeiten und Widersprüche einher. Die Kritiken beziehen sich zum Teil auf das Konzept und die Theorie, die hinter dem Konzept steht, zum Teil aber auch - und das ist oft nicht ganz klar trennbar und trotzdem wichtig zu unterscheiden - auf konkrete Formen, die das Konzept

68 Inwieweit diese Begriffsbestimmungen repräsentativ für die Position des Jugendnetzwerks Lambda BB generell sind, wird nicht expliziert.

69 Jugendnetzwerk Lambda:BB: queer@school.

70 Ebd.

71 Jule Karakayali/Vassilis S. Tsianos/Serhat Karakayali/Aida Ibrahim: »Decolorise it! Die Rezeption von Critical Whiteness hat eine Richtung eingeschlagen, die die antirassistischen Politiken sabotiert«, in: ak - analyse \& kritik (2013), S. 5-8, hier S. $5 \mathrm{ff}$. 
in der Umsetzung in die Praxis angenommen hat. Critical Whiteness oder auch »Kritisches Weißsein« ist der Versuch, den Fokus auf Menschen mit »Privilegien« zu richten und deren Beteiligung an Rassismus - und dessen Aufrechterhaltung - zu benennen. ${ }^{72}$ Der Privilegien-Begriff ist im Zuge der »Theorieproduktion zu Rassismus«, im Anschluss an die Schwarze Bürgerrechtsbewegung in den USA, entstanden. Während einerseits die Meinung vertreten wird, dass die Praxis der »Privilegien«-Benennung notwendig sei, um Rassismus nicht $\mathrm{zu}$ verleugnen und unsichtbar $\mathrm{zu}$ machen, und dass diese Form der Reflexion in der konkreten Praxis wirkungsvoll gegen Rassismus sei, wird andererseits die Frage gestellt, was es zu gewinnen gebe, wenn Menschen in Bezug auf ihre verschiedenen Identitäts-Kategorien jeweils auf die Plätze »privilegiert « oder »diskriminiert« verwiesen werden. Zudem affirmiere, so die Kritik, die Verhältnisbestimmung von "Privilegien« und »Diskriminierungen« als ein Nullsummenspiel eine neoliberale Konkurrenz-Logik. Das erschwere auch eine Politik der gemeinsamen Interessen. ${ }^{73}$ Es wird in Frage gestellt, ob es für eine antirassistische Praxis notwendig sei, »politische Analysen und theoretische Zugänge an die Herkunft von Menschen« zu binden, und die Befürchtung geäußert, dass dabei das Politische als Ort des »Dissens« verloren gehe. Diesen Kritiken wird wiederum entgegengesetzt, dass Positionierungen nicht per se essentialisierend seien, sondern dass es sich um historisch gewachsene »Positionen im Kontext « von Rassismus handele, die auch Koalitionsarbeiten nicht entgegenstehen würden. ${ }^{74}$ Auch hier greift das Argument der Notwendigkeit, auf real wirkende rassistische Strukturen aufmerksam zu machen. Die Vision, die hinter der »Privilegien«-Praxis steht, kann diesen Debatten folgend insofern als eine offene verstanden werden, als es darum geht, Koalitionen möglich zu machen, in denen Möglichkeiten und Ressourcen politischer und gesellschaftlicher Teilhabe miteinander geteilt werden, aber auch insofern, als die Auflösung der Kategorie race angestrebt wird. Eine geschlossene Praxis ist sie aber insofern, als Kategorien, die auf einen Platz verweisen, sowie mitunter das Nullsummenspiel neoliberaler Logiken affirmiert werden. In Bezug auf die verschiedenen Formen, die Critical Whiteness in der konkreten Praxis annehmen kann, wird zudem kritisiert, dass sie zu einem »Wettbewerb der Unterdrückten« oder der »Weißen«, die sich als "gute solidarische Subjekte ins Rampenlicht stellen«, führen kann. ${ }^{75}$ Politiken im Namen von Critical Whiteness wird aber auch vorgeworfen, »moralisierend« zu sein oder bewusst Gefühle der »Schuld« und »Scham« bei den

72 Der Privilegien-Begriff wird nicht immer in einer »Nullsummen-Logik« verwendet. Es gilt, sich jeweils genau anzuschauen, wie er verstanden wird.

73 Vassilis S. Tsianos/Jule Karakayali/Sharon D. Otoo/Joshua K. Aikins/Serhat Karakayali: »Dimensionen der Differenz. Ein Cespräch über Critical Whiteness und antirassistische Politik zwischen Vassilis Tsianos, Juliane Karakayali, Sharon Dodua Otoo, Joshua Kwesi Aikins und Serhat Karakayali«, in: ak - analyse \& kritik (2013), S. 11-18, hier S. 11.

74 Ebd., S. 14.

75 Bee, Melanie: Das Problem mit >Critical Whiteness،. Migrazine 2013, https://www.migrazine.at/art ikel/das-problem-mit-critical-whiteness vom 23.11.2020. 
»Privilegierten« hervorzurufen. ${ }^{76}$ Zudem sei es im Namen von CW nur noch möglich, " ExpertIn< seiner/ihrer selbst zu sein «. ${ }^{77}$

Wenn das Jugendnetzwerk Lambda BB als »privilegierte mehrheitsweiße« Gruppe vom CSD fordert, den Blick verstärkt auf mehrfachmarginalisierte Vereine zu richten, positioniert sich die Einrichtung in einem Critical-Whiteness-Diskurs, der zum einem umkämpft ist und zum anderen sehr unterschiedliche Formen annehmen kann. Zunächst einmal fordert Lambda BB im Namen dieser Praxis Solidarität mit und Einschluss von mehrfachmarginalisierten Gruppen. Damit setzt sich die Einrichtung für eine Öffnung des CSD ein und konstituiert sich selbst als eine solidarische Gemeinschaft. Gleichzeitig scheint Lambda BB im Namen einer Politik, in der Privilegien verantwortungsvoll geteilt werden, das dadurch legitimierte »Rampenlicht ${ }^{78} \mathrm{zu}$ nutzen, um primär über die eigene Arbeit zu sprechen. Lauren Berlant verweist auf eine mögliche Widersprüchlichkeit, die mit den »mitfühlenden Praktiken subalterner Zeug*innenschaft und Anklage» einhergeht:

》Öffentlich zur Schau gestellte Sentimentalität präsentiert sich als kollektive Weigerung, das kollektive Leid eines Teils der Bevölkerung noch länger zu ertragen, de facto stellt sie jedoch allzu oft eine defensive Reaktion von Leuten dar, die sich mit Privilegien identifizieren, zugleich jedoch fürchten, als unmoralisch dazustehen, weil sie einer bestimmte Form struktureller Gewalt, aus der sie einen Nutzen ziehen, stillschweigend zustimmen. ${ }^{79}$

Lambda BB positioniert sich innerhalb eines Critical-Whiteness-Diskurses, indem in Bezug zu Identitäts-Kategorien stehende Privilegien benannt und mit der politischen Aufforderung verknüpft werden, sich für den Einschluss von Mehrfachdiskriminierung einzusetzen. Mit dieser Haltung versucht Lambda BB, eine offene, solidarische Bündnispolitik zu betreiben. Dieser Versuch läuft aber gleichzeitig auch Gefahr, in die damit einhergehenden »Fallen« zu tappen und anderweitig Schließungen zu produzieren. Diese Politik wird zudem im Namen dessen, was bei »Stonewall« wirklich passiert sei - der »richtigen« Geschichte - , legitimiert; diese wird so erneut festgeschrieben.

Auch TransInterQueer e.V. erinnert an eine bestimmte Geschichte, wenn die Einrichtung Ja zum CSD sagt. Auch im Fall von TrIQ gibt es nur eine komplizierte Antwort auf die

76 Dem steht wiederum das Konzept der»White Fragility « entgegen, dass eine Reaktion weißer Menschen beschreibt, die nicht damit umgehen können, dass Ihnen Rassismus vorgeworfen wird. Indem sie den Fokus auf die eigenen Gefühle, die durch die Kritik an deren rassistischen Praktiken verletzt wurden, richten, tragen sie dazu bei, dass erneut wieder nur über »weiße« Menschen gesprochen wird. DiAngelo, Robin: White Fragility. Why It's So Hard for White People to Talk About Racism, Boston: Beacon Press 2018.

77 V. S. Tsianos/J. Karakayali/S. D. Otoo/J. K. Aikins/S. Karakayali: Dimensionen der Differenz.

78 Sie können nur mit gutem Gewissem Ja zum CSD sagen, wenn sie es im »Ceiste von Stonewall« im Einsatz für »Mehrfachdiskriminierte«tun.

79 Lauren Berlant: »Das Subjekt wahrer Gefühle: Schmerz, Privatheit und Politik«, in: Angelika Baier/Christa Binswanger/Jana Häberlein et al. (Hg.), Affekt und Geschlecht. Eine einführende Anthologie, Wien: Zaglossus Verlag 2014, S. 87-117, hier S. 88. 
Frage, wie sich das Verhältnis von Identität und der Konstruktion der eigenen politischen Gemeinschaft im Kontext des CSD artikuliert. TrIQ fordert zunächst im »Geiste« seiner Geschichte einen CSD der Bündnispolitiken oder auch »Allies«. Bündnisse oder auch »Koalitionen des Überlebens« - wie Sabine Hark sie nennt - gelten als Möglichkeiten der Politik jenseits identitätspolitischer Schließungen und neoliberaler Logiken. ${ }^{80}$ Diese »Koalitionen des Überlebens« werden als Bündnisse, in denen im Namen von Trans* und Inter* ein Ende von rechtlicher und medizinischer Normierung gefordert wird, näher bestimmt. Diese Bestimmung enthält zunächst einmal vermeintliche Identitäts-Kategorien, wie Trans* und Inter*. Im »Trans* Inter* Queer ABC« definiert TrIQ Trans* und Inter* als Begriffe, die nicht zuletzt aufgrund der Offenheit, die das Sternchen darstellt, die Vielfalt trans* - und inter*-geschlechtlicher Lebens- und Seinsweisen umfassen. ${ }^{81}$ Das, was das Trans* - oder Inter*-Spezifische dieser Vielfalt ist, wird nur zirkulär über die Begriffe Trans" - und Inter*-Geschlechtlichkeit erklärt. TrIQ spricht von der Vielfalt inter*-geschlechtlicher Realitäten beziehungsweise Körperlichkeiten und trans*-geschlechtlicher und anderer, nicht der Norm entsprechender Geschlechtsidentitäten, Selbstbezeichnungen und Lebensentwürfe. Damit bleiben, zumindest in dieser Definition, die Begrifflichkeiten so offen, dass sie streng genommen nichts aussagen, außer dass sie Vielfalt repräsentieren und bei inter* eher Körperlichkeiten und Realitäten, bei trans* eher Geschlechtsidentitäten, Selbstbezeichnungen und Lebensentwürfe eine Rolle spielen. Die Bezeichnungen, in deren Namen zukünftige Koalitionen gefordert werden, werden also von TrIQ selbst nicht beziehungsweise nicht nur als Identitäts-Kategorien verstanden.

Bündnisse werden in den Forderungen von TrIQ aber auch durch das Ziel - Gleichheit und Anerkennung vom Staat zu fordern - näher bestimmt. Auf Berlants Ausführungen Bezug nehmend, kann davon gesprochen werden, dass in diesen Forderungen der "Staat und das Recht als zentraler Hort des Personseins« bestätigt wird. Der Staat gilt, queer-theoretischen Überlegungen zufolge, selbst auch als Ort, der ungleiche Verteilungen und damit Ungerechtigkeiten organisiert. ${ }^{82}$ Forderungen an den Staat laufen Gefahr, sich dem Staat als dem Ort, der Ungerechtigkeiten organisiert, zu unterwerfen oder zumindest den Staat als den Ort anzuerkennen, der bis zu einem gewissen Grad darüber entscheidet, wie Ungerechtigkeiten verteilt werden. ${ }^{83}$ Auf der einen Seite nehmen sich Gruppen, die Recht und Schutz durch den Staat einfordern, die Möglichkeiten der eigenen Handlungsmächtigkeit ${ }^{84}$ und bejahen einen »libertären « oder »autoritären« Neoliberalismus, der über das Versprechen sexueller Freiheiten oder Sicherheiten fungiert. ${ }^{85}$ Auf der anderen Seite wird ja gerade weniger autoritative/hierarchische Abhängigkeit von staatlichen Institutionen eingefordert. Insofern geht es auch

80 Vgl. S. Hark: Koalitionen des Überlebens.

81 TransInterQueer e.V.: Trans*Inter*Queer*ABC.

82 L. Berlant: Das Subjekt wahrer Gefühle: Schmerz, Privatheit und Politik, S. 88.

83 Judith Butler/Gayatri C. Spivak: Sprache, Politik, Zugehörigkeit, Zürich: Diaphanes 2011, S. 8.

84 J. Butler: Haß spricht, S. 37.

85 Gundula Ludwig/Volker Woltersdorff: „Sexuelle Politiken im autoritären Neoliberalismus zwischen den Versprechen von Freiheit und Sicherheit«, in: Katharina Pühl/Birgit Sauer (Hg.), Kapitalismuskritische Gesellschaftsanalyse: queerfeminstische Positionen, Münster: Westfälisches Dampfboot 2018, S. 47-72, hier S. 54ff. 
darum, bestimmte Grenzlinien, entlang derer die Möglichkeiten gesellschaftlicher Teilhabe verlaufen, zu öffnen (aufzubrechen). Allerdings schließen solche Forderungen nur jene Menschen ein, die Rechtssubjekte sind und Anspruch auf medizinische Versorgung erheben können. Indem TrIQ Ja sagt zu einer zukünftigen Koalition im Namen der Geschichte, sagt die Einrichtung Ja zu Bündnispolitiken, die ein »öffnendes« Potential haben. Gleichzeitig setzt auch TrIQ im Namen der Geschichte schwul-lesbischer Kämpfe fest, um welche Forderungen diese Bündnispolitiken angeordnet sind. Sowohl die Festsetzung als auch die Forderungen selbst bringen bestimmte Momente der Schließung mit sich. ${ }^{86}$

In der »Out! « des Jugendnetzwerks Lambda wird das Verhältnis zwischen der eigenen Gemeinschaft und dem CSD jenseits der expliziten Verwendung von Identitäts-Begriffen hergestellt. Auf den Maßstab des Grades der Schließung rekurrierend, scheint die in Bezug auf den CSD hergestellte - politische Gemeinschaft vermeintlich offen, kann doch jede* $r$ in Bezug auf den CSD entscheiden, wie er*sie will. Im Sprechen von individueller Entscheidung und Verantwortung klingt jedoch unhinterfragt ein neoliberaler Imperativ an. Es wird kein kollektives Wir um ein gemeinsam geteiltes Verhältnis zum CSD hergestellt - sondern ein Wir, das aus vielen, für sich selbst verantwortlichen Individuen besteht. Die Ausschlüsse derjenigen »Leben«, die aufgrund der »ungleichen Verteilung von Gefährdetheit « vermehrt einer solidarischen Gemeinschaft bedürfen, sind in solch einer individualistischen Konstruktion von Gemeinschaft konstitutiv. ${ }^{87}$ Eine individuelle Entscheidung kann nicht jede Person auf gleiche Weise treffen. Manche Personen sind mit Butlers Worten gefährdeter als andere und bedürfen eher der Unterstützung einer Gemeinschaft, um auf der Straße erscheinen zu können. ${ }^{88}$ Das, was hier hergestellt wird, ist eine vermeintliche Gemeinschaft, die insofern offen ist, als alle sich zum Teil widersprechenden, unterschiedlichen Entscheidungen in Bezug auf den CSD als Teil der Lambda-Gemeinschaft getroffen werden können. Allerdings können diese Entscheidungen nur »individuell« und eben nicht als Gemeinschaft getroffen werden. Damit werden neoliberale Logiken, denen zufolge »jede" $r$ « für sich selbst verantwortlich ist, auf Kosten solidarischer Politiken, die auf einer wechselseitigen Abhängigkeit voneinander beruhen, bejaht.

Im Gegensatz zu dieser individualistischen Konstruktion von Gemeinschaft bejaht die Türkis Rosa Lila Villa eine Regenbogenparade, die nicht alle repräsentiert, indem sie solidarisch und in Kooperation mit anderen Projekten einen eigenen, alternativen Raum für die Nicht-Repräsentierten auf der Parade herstellt. In der und durch die Zusammenarbeit und Kooperation mit anderen Projekten ordnen sich die CSD-Politiken um eine queere Vision von Koalitionen an. Analog zu dem, was Muñoz als disidentification bezeichnet - nämlich "gleichzeitiges Arbeiten an, mit, und gegen« dominante Anrufungen -, arbeitet die Villa zunächst mit der Anrufung der Regenbogenparade, indem sie

86 J. Butler: Das Unbehagen der Geschlechter, S. $34 \mathrm{f}$.

87 J. Butler: Anmerkungen zu einer performativen Theorie der Versammlung, S. 196.

88 Vgl. ebd. 
teilnimmt. ${ }^{89}$ Damit bejaht sie zunächst auch die durch die Praktiken der Regenbogenparade produzierten Ausschlüsse. Gleichzeitig arbeitet die Villa jedoch auch "gegen« die ausschließenden Mechanismen der Parade, indem die Einrichtung sich nicht vollständig mit der Anrufung der Regenbogenparade »identifiziert«, sondern die aus der Anrufung Ausgeschlossenen hereinzuholen versucht, indem ein alternativer Raum ein "queer-feministischer antirassistischer Truck« - hergestellt wird. Die Parade wird umgearbeitet, anstatt die durch die Parade produzierten Schließungen vollständig anzunehmen. Anders als andere Pride-Paraden gilt die Wiener Regenbogenparade allerdings als ein Ort, an dem es keine streng kontrollierte Ordnung gibt und Gruppen sich relativ »frei bewegen« und anordnen können:

»Bis auf ein paar marginale Sicherheitsmaßnahmen, wie in Bezug auf Mindestseitenabstände zu den großen Trucks, können sich die Individuen frei bewegen. Dies geht sogar so weit, dass einzelne aktivistische Cruppen, die ihre Teilnahme aus unterschiedlichen Gründen nicht offiziell anmelden wollen, sich zu anderen Gruppen ihrer Wahl hinzugesellen und mit ihren eigenen Transparenten, Slogans und Performances die Parade bereichern können ${ }^{90}$

Die Villa sagt also Ja zu einer ausschließenden Parade, um selbst auf der Straße an den ausschließenden Grenzen der Regenbogenparade zu arbeiten. Dieses Ja ist aber nur aufgrund der spezifischen Anordnungen der Regenbogenparade in Wien möglich.

In der Art und Weise, wie die einzelnen Projekte sich in der Verhandlung ihres eigenen Verhältnisses zur Pride als kollektives Wir konstituieren, zeigt sich, dass alle Projekte bestimmte Kritiken an (identitätspolitischen) Schließungen ernst nehmen und versuchen, darauf zu antworten. Vor allem in der Weise, wie vermeintliche IdentitätsKategorien verwendet werden, hat es eine Verschiebung gegeben. So wird zumeist von »Mehrfachidentitäten « gesprochen, die nicht festschreiben, »wer wir sind«, sondern "wie wir in Machtverhältnisse verstrickt sind«, oder es werden Begriffe verwendet, die als »Sammelbegriffe« für die »Vielfalt« verschiedenster Identitäten und Körperlichkeiten stehen. Verwendet werden diese vermeintlichen Identitäts-Kategorien, um eine Öffnung des CSD für die bezeichneten Gruppen einzufordern (Solidarisierung mit Mehrfachdiskriminierten, Unterstützung von Trans*- und Inter*-Kämpfen). Um der neoliberalen Vereinnahmung von vielfältigen Identitäten zu entkommen, werden Affekte und Körper statt Identitäten als Mittel und Zweck des Politischen aufgerufen. Gemeinsam ist allen Aushandlungen, dass der CSD ein offener Ort jenseits von (rassistischen und kommerzialisierten) Ausschlüssen sein soll. Oft wird allerdings in Namen dessen, was der CSD eigentlich ist, ein offenerer CSD gefordert. Dabei wird häufig auf eine bestimmte Art und Weise, den CSD zu erinnern, rekurriert und im Namen einer anderen, wahren »Stonewall Inn«-Geschichte ein weniger ausschließender und kommerzialisierter CSD gefordert. 


\subsection{Mehrfachdiskriminierung und Rassismus: Wir sind queer - Wir sind solidarisch}

Die offizielle queere Geschichte beginnt nicht mit »Stonewall«, auch wenn, wie in der vorherigen Analyse zur Pride versucht wurde zu zeigen, queere Politiken den CSD als Erinnerung an »Stonewall« und damit als Ort für queere Politiken einfordern. Im Gegensatz zu den oft als identitätspolitisch geltenden schwul-lesbischen Politiken des Stolzes, die »Stonewall« repräsentiert, wird der Anfang von »queer« in der Aids-Krise und den damit einhergehenden Bündnispolitiken verortet. Die Analysen zur Positionierung in Bezug auf die Pride verweisen bereits auf die zentrale Rolle queerer Bündnisse. Wie sehen aber solche Bündnisse aus, nachdem der Kampf gegen den Tod für viele nicht mehr derart zentral ist? ${ }^{91}$ Wie lassen sich Bündnisse begründen, die nicht durch ein gemeinsames identitätspolitisches Band zusammengehalten werden? Im Materialkorpus ist die Frage nach queeren Bündnissen und Koalitionen maßgeblich gekoppelt an Diskurse um Mehrfachdiskriminierungen. Besonders präsent ist in diesem Zusammenhang bei (fast) allen Projekten die Verhandlung von Mehrfachdiskriminierung im Kontext von Rassismus. Die Art und Weise, wie Bündnispolitiken auszusehen haben, wird maßgeblich darüber verhandelt, wie die jeweilige politische Gemeinschaft Mehrfachdiskriminierungen vor allem in Bezug auf Rassismus thematisiert. Das kollektive Wir wird über eine bestimmte Art und Weise, Mehrfachdiskriminierung zu thematisieren, hergestellt. Gemeinsam ist dabei allen Projekten, dass sie sich in und durch einen bestimmten Umgang mit Mehrfachdiskriminierung als solidarisches Wir konstituieren. Die Thematisierung beziehungsweise der Umgang mit Mehrfachdiskriminierung findet dabei auf verschiedenen Ebenen statt. Zunächst geht es darum, den Rassismus sowie die Leerstellen und Widersprüche im Kontext von Rassismus innerhalb der eigenen Gemeinschaft zu hinterfragen und zu reflektieren und einen entsprechenden Umgang damit auszuloten. Gleichzeitig - und das ist von der Auseinandersetzung mit Rassismus und Mehrfachdiskriminierung in der eigenen Gemeinschaft nicht zu trennen - geht es auch darum, die Leerstellen, Widersprüche und rassistischen Praktiken »außerhalb« der eigenen Gemeinschaft (z.B. in der Community, Gesellschaft, Politik oder medialen Berichterstattung) zu benennen und »Solidarität « von ebendiesen einzufordern. Von den anderen Solidarität einzufordern, ist dabei wiederum auch eine Form, sich als solidarisches Wir zu konstituieren. Sich im Kontext von Mehrfachdiskriminierung als solidarisches Wir zu konstituieren, bedeutet in den diskursiven Aushandlungen der fünf queeren Projekte, die eigenen problematischen, ausschließenden Praktiken zu benennen und an ihnen zu arbeiten. Es bedeutet aber auch, die ausschließenden Praktiken der anderen zu benennen und von ihnen Solidarität einzufordern. Dabei schließen die Thematisierungen von Mehrfachdiskriminierung, insbesondere von Rassismus, durch die fünf Projekte an bestimmte, in deutschsprachigen (queeren) Kontexten dominante Diskurse und Debatten an. Spätestens mit der Ablehnung des Zivilcourage-Preises durch Judith Butler am CSD 2010 wurde eine Debatte um Rassismus in vielen Teilen

91 Zumindest für die Mehrheit der westlichen queeren Community ist der Kampf gegen den Tod nicht mehr derart zentral. Das gilt aber nicht für alle. Trans*-Personen, Sexarbeiter*innen und Queers of Color sind auch im Westen besonders gefährdet, Opfer von Hate Crime zu werden. 
der deutschsprachigen queeren Communitys angestoßen. Auch diese Debatten nehmen vorwiegend Bezug auf Interventionen aus den USA, wie sie im Gefolge der Schwarzen Bürgerrechtsbewegungen entstanden sind. ${ }^{92}$ Der in dem Analysematerial häufig verwendete Begriff »Mehrfachdiskriminierung«, aber generell auch die Art, wie versucht wird, die intersection von verschiedenen Unterdrückungssystemen in den Blick zu nehmen, verweist darauf, dass intersektionale Perspektiven konstitutiver Bestandteil aktueller queerer Diskurse sind. Auch verschiedene postkoloniale Interventionen und Critical-Whiteness-Perspektiven sind zentrale Diskurspositionen, die die untersuchten Debatten zum Thema Mehrfachdiskriminierung prägen.

Je nach Themen- und Arbeitsschwerpunkt, aber auch abhängig von der Rolle, die Rassismus in der jeweiligen Community spielt, findet die Auseinandersetzung mit Rassismus innerhalb und außerhalb der jeweiligen Projekte mehr oder weniger intensiv statt - sie wird aber in allen Projekten geführt. Nachdem der Arbeitsschwerpunkt von LesMigraS in der Antigewalt- und Antidiskriminierungsarbeit mit Fokus auf Mehrfachdiskriminierung liegt, hat das Thema Rassismus einen zentralen Stellenwert in der Bewegungsöffentlichkeit der Einrichtung. So trägt die jahrelange Präsenz und Aufklärungsarbeit von LesMigraS und anderen Gruppen dazu bei, dass vor allem in der Berliner Community das Thema Rassismus besonders präsent ist. Entsprechend positionieren sich alle drei Berliner Gruppen (TrIQ e.V., Lambda BB und LesMigraS) regelmäßig zum Thema Rassismus, vor allem im Rahmen von Auseinandersetzungen in der Berliner Community. In der Villa sind Debatten um Rassismus wohl auch insofern präsent, als sich seit den 2000 er-Jahren verschiedene queere "migrantische« Gruppen in den Räumen der Villa gegründet haben. Das seit 2008 bestehende "FreiRäumchen ", ${ }^{93}$ das regelmäßig in der Villa stattfindet, gilt als der Austauschort und Treffpunkt für viele Queers of Color, "geflüchtete« und "migrantische« Queers. Entsprechend positioniert sich auch die Villa schon seit Beginn des Analysezeitraums (2010) zu Mehrfachdiskriminierung und Rassismus, vor allem in Bezug auf Flucht und Asyl. Flucht und Asyl wird besonders mit der Gründung des Vereins "Queer Base«, dessen Ziel es ist, sicheren Wohnraum für queere Geflüchtete zu schaffen, im Jahr 2014 relevant. Auch im »Milchbüechli« kommen Auseinandersetzungen mit Rassismus vor, allerdings haben sie nicht die gleiche Relevanz wie in den anderen Projekten. Hin und wieder gibt es Kommentare, in denen vor allem die eigenen rassistischen Ausschlüsse thematisiert werden. Im Vergleich zu den anderen Projekten, aber auch den anderen Themen im »Milchbüechli« hat das Rassismus-Thema allerdings einen geringeren Stellenwert. Auch in der Jugendzeitschrift des Jugendnetzwerks Lambda werden rassistische Ausschlüsse kaum bis gar nicht thematisiert. Im Gegenteil, hier zeigt sich besonders in der Rubrik »Denkwürdiges« eher ein Diskurs des Othering.

Nachdem die Kritik an rassistischen Praktiken des CSD durch LesMigraS im vorherigen Abschnitt bereits detaillierter beleuchtet wurde, wurden für diesen Analyseab-

92 In Kapitel eins ist eine Darstellung der Interventionen Schwarzer Feminist*innen und postkolonialer Perspektiven zu finden.

Das »FreiRäumchen« wird von den Villa-Aktivist*innen getragen. 
schnitt die Auseinandersetzungen von zwei (mehrheitsweißen) ${ }^{94}$ Projekten für die feinanalytische Darstellung gewählt. Beide Diskursfragmente wurden ausgewählt, weil sie repräsentativ für die Auseinandersetzung der jeweiligen Projekte sind und weil sie an jeweils ein diskursives Ereignis anknüpfen. Zum einen wurde der Artikel »Abartige gegen Abschiebung “" ${ }^{95}$ der Türkis Rosa Lila Villa ausgewählt. Der Artikel ist insofern für die Villa-Diskurse repräsentativ, als es sich bei Rassismus und Flucht um das am meisten verhandelte Thema in den Villa-Texten handelt. Zudem wird die Überschrift - »Abartige gegen Abschiebung «-, die gleichzeitig auch Aufhänger des Artikels ist, immer wieder in Öffentlichkeitskontexten der Villa verwendet. Ursprünglich war es die Aufschrift auf einem Banner, das 2010 von der Hausfassade hing; im Artikel wird dieses Statement erneut aufgegriffen, um über das Projekt »Queer Base« zu berichten. Das Banner ist auch auf dem Bild des zuvor analysierten CSD-Berichtes zu sehen und die Perverse Partei Österreichs verwendet den Slogan für die eigenen Plakatkampagnen. Der 2015 erschienene Text schließt auch an die gesellschaftspolitischen Debatten um die sogenannte »Flüchtlingswelle« im Jahr 2015 an, die in diesem Zeitraum zu einem medialen diskursiven Ereignis wurden. ${ }^{96}$ Das zweite Feinanalysebeispiel ist eine Pressemitteilung des Jugendnetzwerks Lambda BB, die Bezug nimmt auf eine in der Berliner Community sehr kontroverse Debatte um das Lesbisch-schwule Stadtfest. Die Pressemitteilung wurde ausgewählt, weil diese Debatte so etwas wie ein diskursiver Knotenpunkt ${ }^{97}$ in der Auseinandersetzung mit rassistischen und transphoben Ausschlüssen im Analysezeitraum in der Berliner Community ist. ${ }^{98}$ Alle drei Berliner Projekte - und das ist für den Analysezeitraum einzigartig - positionieren sich zu den Auseinandersetzungen um das Lesbisch-schwule Stadtfest (wenn auch in unterschiedlichen Jahren). Auch in queeren und linken Medien ist und war die Auseinandersetzung in Berlin präsent. ${ }^{99}$ Für das Jugendnetzwerk Lambda BB ist die Pressemitteilung auch insofern repräsentativ, als die Solidarität mit nicht-mehrheitsweißen Gruppen - wie sie es bezeichnen - neben den eigenen Kämpfen um Räume und finanzielle Ressourcen das zentrale Thema der Stellungnahmen von Lambda BB ist. Die beiden Diskursfragmente wurden auch deshalb für die Feinanalyse ausgewählt, weil sich in ihnen zeigt, dass trotz Bezugnahme auf ähnliche Begrifflichkeiten (z.B. Solidarität, Privilegien) und gleiche (theoretische) Diskurse (Critical Whiteness, Intersektionalität, postkoloniale Theorien) die Art und Weise, wie sich das jeweilige Wir in der Auseinandersetzung mit Mehrfachdiskriminierung als solidarisch konstituiert, unterschiedlich ist.

Die Villa versteht sich zumindest historisch als mehrheitsweißes Projekt und spricht im Text von vielen mehrheitsweißen Aktivist*innen, die Teil des Projektes sind. Die Villa bezeichnet sich aber selbst nicht als mehrheitsweiße Organisation.

95 Türkis Rosa Lila Villa: Abartige gegen Abschiebung, https://dievilla.at/blog/abartige-gegen-abschi ebung/ vom 14.09.2020.

96 LesMigraS und die Villa, also die beiden Projekte, die Flucht und Asyl thematisieren, sprechen schon lange vor der medialen Präsenz des Themas über Flucht und Asyl. 


\subsubsection{Abartige gegen Abschiebung}

In der Türkis Rosa Lila Villa hat nicht zuletzt auch aufgrund der Gründung des »FreiRäumchens« im Jahr 2008 und des Projekts »Queer Base« im Jahr 2014 das Thema »Rassismus« einen zentralen Stellenwert. Die Villa nutzt seit 2014 vermehrt die eigene Öffentlichkeit (»Lambda-Nachrichten«, Homepage, Hausfassade), um auf die notwendige Unterstützung von »Queer Base« aufmerksam zu machen. Aus diesem Grund wurde vor allem im Jahr 2015 Rassismus im Kontext von Flucht und Asyl häufig thematisiert. So wurde die im Zuge des »Tötet Schwule«-Hatespeech auf der Villa-Hauswand entstandene öffentliche Aufmerksamkeit 2014 genutzt, um auf »Queer Base« aufmerksam zu machen. ${ }^{100}$ Auch der Mord an der türkischen Trans*-Person Hande Öncu, die zu Beginn des Jahres 2015 bei der Sexarbeit ermordet wurde - mit der sie unter anderem ihre Wohnung finanzierte -, wurde politisiert, um Unterstützung für das Wohnprojekt "Queer Base« zu fordern. ${ }^{101}$ Gleichzeitig ist 2015 das Jahr, in dem - aufgrund der sogenannten »Flüchtlingskrise« - dem Thema »Flucht und Asyl« medial und politisch besondere Aufmerksamkeit zukam. In Österreich haben beispielsweise Berichte über die sogenannte »Willkommenskultur« am Wiener Hauptbahnhof, aber auch über siebzig Tote in einem Lkw in Parndorf im August sowie Diskussionen um einen Grenzzaun in Spielfeld die mediale Öffentlichkeit dominiert. In dieses Jahr 2015 fällt auch das für die Feinanalyse ausgewählte Diskursfragment mit dem Titel »Abartige gegen Abschiebung «. ${ }^{102}$ Der Artikel wurde im Mai 2015 auf der Villa-Homepage im Villa-Blog unter der Rubrik »Asyl« veröffentlicht. Am Ende des Artikels wird darauf verwiesen, dass der Artikel kurz zuvor bereits im »Hinterland « erschienen sei, dem »Vierteljahresmagazin des Bayrischen Flüchtlingsrats « (Heft 28/15). ${ }^{103}$ Die Autor*in des Artikels veröffentlicht häufiger Beiträge zur Villa-Politik in externen Online-Zeitschriften. Damit trägt sie maßgeblich zur medialen Außendarstellung der Villa bei. Der auf der Homepage veröffentlichte Artikel ist dem im »Hinterland« veröffentlichten Artikel sehr ähnlich. Im »Hinterland«-Artikel sind die Sätze oft etwas kürzer, es gibt mehr Absätze und diese sind zum Teil mit Überschriften versehen, die auf der Homepage-Veröffentlichung weggefallen sind. Beide Versionen beginnen allerdings mit der Überschrift »Abartige gegen Abschiebung «, die auf ein Banner Bezug nimmt, das 2010 die Hausfassade der Villa bedeckte.

Ein Bild, das sich unter dem ersten Absatz des Artikels auf der rechten Seite befindet, zeigt die damalige Hausfassade mit dem ca. 20 Meter langen Transparent. Die Unterüberschrift des Artikels - »Solidarität mit LGBTIQ Flüchtlingen« - verweist auf die politische Haltung, die mit dem Banner und seinem Aufdruck ausgedrückt werden

100 Türkis Rosa Lila Villa: Schatzi, wir lassen uns nicht einschüchtern!

101 Türkis Rosa Lila Villa: »Zum Mord an Hande Öncü«, in: Lambda-Nachrichten, https://lambdanachr ichten.at/In1501.pdf vom 24.11.2020, S. 18.

102 Türkis Rosa Lila Villa: Abartige gegen Abschiebung.

103 Marty Huber: »Abartige gegen Abschiebung«, in: Hinterland, https://www.hinterland-magazin .de/wp-content/uploads/2016/11/hinterland-magazin-28-68-abartige-gegen-abschiebung.pdf vom $17.20 .2020,2015$, S. 68-69. 
soll. Der Artikel ist in sieben Abschnitte unterteilt, die formal durch Absätze gekennzeichnet sind. Zwischen dem ersten und dem dritten Abschnitt und neben dem zweiten Abschnitt befindet sich ein Bild der Villa-Hausfassade mit dem Banner »Abartige gegen Abschiebung«. Auf dieses Banner, dessen Statement zugleich die Überschrift des Artikels darstellt, nimmt der erste Abschnitt Bezug.

Im ersten Abschnitt wird zunächst das Banner aus dem Jahr 2010 beschrieben und über die Abschiebung eines nigerianischen Geflüchteten als Anlass für den Aufdruck auf dem Banner gesprochen.

Der Abschnitt beginnt mit der Beschreibung des Banners, dessen Motiv zugleich Überschrift und Aufhänger des Artikels ist: »ein Portrait der damaligen ÖVPInnenministerin Maria Fekter und die betenden Hände Albrecht Dürers von denen Blut tropfte. Neben den Worten >Maria Frontex war der Spruch >Abartige gegen Abschiebungく zu lesen.« Der Beschreibung und Erklärung des Banners folgt keine Interpretation. Die Aussage des Banners wird entweder als »selbsterklärend« erachtet oder bleibt bewusst der Interpretation der Leser*innen überlassen. Was sind nun aber die Aussagen des Banners? Welche Verbindungen und Verknüpfungen werden hergestellt und in welchem Bezug stehen diese zu der Art und Weise, wie die Villa sich im Sprechen über Mehrfachdiskriminierung und Rassismus als politisches Subjekt konstituiert? Zunächst einmal kann die Bezeichnung "Maria Frontex«, in der der Nachname der Innenministerin "Fekter« durch "Frontex« ersetzt wird, als Synekdoche ${ }^{104}$ bezeichnet werden. In dem Fall wird durch den Einsatz der rhetorischen Figur eine semantische Beziehung zwischen »Fekter« und »Frontex", die eigentlich der Synekdoche vorausgehen sollte, hergestellt. Der Eigenname »Maria« (Fekter) - als Repräsentantin der österreichischen Innenpolitik und der »Österreichischen Volkspartei« (ÖVP) - wird verknüpft mit »Frontex«, der Europäischen Agentur für Grenzund Küstenwache, die symbolisch für restriktive Flüchtlingspolitiken steht. Indem der Nachname »Fekter« durch »Frontex« ersetzt wird (beide Begriffe repräsentieren eine bestimmte Politik), wird eine (familiäre) Beziehung zwischen Maria Fekter und dem, wofür »Frontex« steht - nämlich restriktive Flüchtlingspolitik -, hergestellt. Auch die »Betenden Hände« Albrecht Dürers, die im Bild zu Maria Fekters Händen werden, rufen eine bestimmte Kritik an der ÖVP und der österreichischen Innenpolitik auf. Volkstümlich stehen die »Betenden Hände« Dürers für Religiosität. Damit verweisen sie auf den in Alltagsdiskursen oft aufgemachten Widerspruch zwischen der europäischen/österreichischen Asylpolitik einerseits und den christlichen Werten der ÖVP beziehungsweise Österreichs andererseits. ${ }^{105}$ Von den »Betenden Händen « tropft Blut, das auf die vielen Toten, verschuldet durch die österreichische/europäische Asylpolitik, verweist. Die blutenden Hände erinnern auch an die vor allem in queeren

104 Das ist eine rhetorische Figur, in der ein Begriff durch einen anderen aus demselben Begriffsfeld ersetzt wird.

105 Allerdings verweisen die - die christlichen Werte symbolisierenden - Hände gar nicht notwendig auf einen Widerspruch. Die »Betenden Hände« als (Körper-)Teil Maria Fekters fügen sich - denkt mensch beispielsweise an die Rolle der christlichen Missionierung im Zuge der Kolonisierung gut in durch eine christliche Partei forcierte rassistische Politiken, die töten, ein. 
aktivistischen Kreisen bekannte »Bloody Hand«-Kampagne der queeren Gruppe »Act Up«. Mit dieser Kampagne machte »Act Up« in den USA während der Aids-Krise darauf aufmerksam, dass der Staat und die Katholische Kirche »Blut an ihren Händen hat ${ }^{106}$ Die Kampagne wurde auch in Österreich von »Act Up«-Ablegern und der »Anti-ÖVP-Aktion« in den 9oer-Jahren übernommen. Der Slogan wurde etwas umgeschrieben, aber auch damals wurden die ÖVP und die Kirche für die Toten (der Aids-Krise) verantwortlich gemacht: "Die ÖVP und die katholische Kirche haben Blut an den Händen. «107 Mit den blutenden Händen wird auf einer Text-Bild-Ebene eine Verknüpfung zwischen der Kritik an den Aids-Toten und der Kritik an den Toten, die die österreichische/europäische Asylpolitik verschuldet, hergestellt. Der Kampf gegen den Tod derjenigen Leben, die - mit Butler gesprochen - als »nicht betrauerbar« gelten, wird als gemeinsamer Nenner solidarischer Politiken aufgerufen. ${ }^{108}$ Die Toten der Aids-Krise sind jetzt die Toten der Flüchtlingspolitik.

Der Beschreibung des Banners folgt eine Erklärung des Umstands, der 2010 der Anlass zur Herstellung des Banners war: die Abschiebung eines »Flüchtlings« aus Nigeria, der seinen Fluchtgrund »Homosexualität« zu spät im Asylverfahren genannt hat. Durch die Verwendung der Konjunktiv-Form und die Formulierung »nennt sich das « distanziert sich der Artikel von der damaligen Begründung des negativen Asylbescheids: "Außerdem könne er, weil seine Homosexualität noch nicht amtsbekannt sei, innerhalb Nigerias umziehen und sich und seine Neigung verstecken. Innerstaatliche Fluchtalternative nennt sich das und wird besonders gern bei Ländern wie Nigeria, Indien oder Pakistan angewandt.«

Der zweite Abschnitt ist direkt neben dem Bild der Villa-Fassade samt Banner angeordnet. Hier wird berichtet, dass in den letzten Jahren immer mehr »LGBTIQ-Flüchtlinge« Unterstützung in der Villa gesucht hätten, was die Aktivist*innen aufgrund mangelnder Fortbildung und Wissen um das sich ständig ändernde Asylverfahren vor große Herausforderungen gestellt habe.

Zunächst wird die Türkis Rosa Lila Villa als ein Ort beschrieben, an dem »LGBTIQFlüchtlinge« Unterstützung suchen. Gefunden haben zunächst einmal die Aktivist*innen Widersprüche und Leerstellen - ihre eigne Arbeit betreffend: »[E]s wurde für die Aktivist_innen immer klarer, dass [sic!] aufgrund der Komplexität des Asylverfahrens, die in Österreich halbjährlich stattfindenden Verschärfungen [sic!] eine genaue Auseinandersetzung und Fortbildung brauchten.«Die Villa konstituiert sich hier insofern als offener Raum, als Menschen, die noch nicht mitgedacht werden, die Möglichkeit haben, trotzdem Unterstützung anzufordern. Als Antwort auf die Leerstelle, mit der die Villa-Aktivist*innen durch die Forderungen der Geflüchteten konfrontiert wurden, folgen Überlegungen zu einer Öffnung der Unterstützungsmöglichkeiten. Gleichzeitig

106 In den USA symbolisierten blutende Hände an den Wänden eine Kritik an Kirche und Politik, die zu den vielen Toten während der Aids-Krise beitrugen. VgI. L. Hieber: Politisierung der Queer Culture durch ACT UP.

107 U. Repnik: Die Geschichte der Lesben- und Schwulenbewegung in Österreich, S. 158.

108 J. Butler: Raster des Krieges, S. 21. 
positionieren sich die Aktivist*innen »im Kontext freiwilliger und unbezahlter emanzipatorischer Arbeit « und verweisen damit auf die Widersprüchlichkeit, mit der sie, wenn sie auf die »Notwendigkeit« - Unterstützungsmöglichkeiten für LGBTIQ-Geflüchtete zu schaffen - antworten, konfrontiert sind. Mit den Selbstzuschreibungen »freiwillig « und »unbezahlt « wird den Aktivist"innen zunächst ein Stück weit die Verantwortung, auf die »Notwendigkeit « $\mathrm{zu}$ antworten, genommen, indem sie auf mangelnde Ressourcen (»unbezahlte Arbeit«) und implizit auch auf die Aufgabe des Staates - institutionalisierte und professionalisierte Einrichtungen als Gegenspieler zur »unbezahlten«, »freiwilligen« Arbeit - verweisen. Die daran anschließende Selbstbeschreibung als »emanzipatorisch« stellt, im Gegensatz zur »Freiwilligkeit« und »Unbezahltheit«, aber wieder eine (politische) Verantwortlichkeit her. Mit diesen drei selbstbeschreibenden Begriffen werden die Widersprüchlichkeiten im Umgang mit der durch die Geflüchteten eingeforderten »Notwendigkeit« aufgezeigt: Auf der einen Seite ist es Aufgabe des Staates, die Verantwortung auch für LGBTIQ-Geflüchtete zu übernehmen, auf der anderen Seite kann die Villa als emanzipatorisches Projekt nicht Nein sagen zu den angeforderten Unterstützungen und will diese auch nicht dem Staat überlassen. Dafür brauchen die "freiwillig «, »unbezahlt « und »emanzipatorisch « arbeitenden Aktivist*innen selbst Unterstützungsstrukturen, wie Fortbildungen, Ausbildungen und Vernetzungen: „Gerade im Kontext freiwilliger und unbezahlter emanzipatorischer Arbeit ist es besonders wichtig, gut informiert und vernetzt zu sein«. Die Villa stellt sich in diesem Abschnitt als kollektives Wir her, das aus einer emanzipatorischen Verantwortung heraus offen ist für diejenigen, die in den Unterstützungsstrukturen der eigenen Gemeinschaft noch nicht mitgedacht sind. Gleichzeitig benötigt dieses Wir selbst aber auch Unterstützung, um angemessen antworten zu können, weil es selbst unter prekären Bedingungen arbeitet.

Im dritten Abschnitt wird über das Asylverfahren und die besonderen Schwierigkeiten für LGBITQ-Geflüchtete aufgeklärt. Diese müssten - obwohl sie sich zum Teil noch nie geoutet haben - ihren Fluchtgrund glaubwürdig darstellen. Im Anschluss wird auf Leerstellen und Probleme bei der Unterbringung und Betreuung von LGBTIQ-Geflüchteten eingegangen: Diese erfahren und erleben in den Unterkünften psychische und physische Gewalt, die von den Institutionen mitgetragen werde.

Im dritten Abschnitt wird zunächst über die Schwierigkeiten im Asylverfahren berichtet, um im Anschluss die Asylunterkünfte als potentielle Ressourcen, aber auch als potentiell negative Verstärker im Asylverfahren festzuschreiben. Zunächst wird von den Anforderungen eines Asylantrags berichtet, in dem "glaubwürdig der eigene Fluchtgrund « geschildert werden muss. Hier wird auf die spezifische Schwierigkeit verweisen, dass gerade für Geflüchtete, die bisher ihre Sexualität oder Geschlechtsidentität verstecken mussten, das »Coming-out «, das der Asylantrag erfordert, eine schwierige Aufgabe ist. Der Artikel spricht aber auch explizit von Geflüchteten, die sehr »klar und spezifisch « in der Schilderung eines Fluchtgrundes seien. Dieser Verweis ist ein Versuch, keinen Gegensatz zwischen den »stolzen geouteten « Homosexuellen und Trans*Menschen im toleranten Europa und den ungeouteten Geflüchteten aus dem intoleranten "globalen Süden« herzustellen. Trotzdem sollen in diesem Abschnitt die spezifischen Dimensionen der Mehrfachmarginalisierung von LGBTIQ-Geflüchteten betont werden. Im zweiten Teil dieses Abschnitts - der in der ersten Version (»Hinterland«- 
Artikel) durch einen Absatz markiert ist - wird dann von der Unterbringungs- und Betreuungssituation gesprochen, die ebenfalls spezifische Schwierigkeiten für LGBTIQGeflüchtete mit sich bringt. Die Überleitung vom Asylverfahren zur Betreuungssituation wird hergestellt, indem das Asylverfahren im Gegensatz zur Wohnsituation als etwas beschrieben wird, das sich mit der Zeit gebessert habe: »Während sich nämlich manche gesetzlichen Voraussetzungen in den letzten Jahren verbessert haben und etwa nicht mehr mit der Begründung >man könne ja die eigene Homosexualität verstecken abgeschoben werden darf, sieht es bei der Unterbringung und Betreuung von LGBTIQ Flüchtlingen schlecht aus.« Hier wird ein Unterschied zwischen dem Asylverfahren, in dem sich Dinge verbessert hätten, und der Unterbringungssituation, die nach wie vor unverändert sei, gemacht, um die Wohnsituation als zentralen Ort der Intervention festzuschreiben. In Bezug auf die Unterbringungssituation wird, an eine Formulierung von Judith Butler anschließend, davon gesprochen, dass die »Gefährdung« der LGBTIQ-Geflüchteten - explizit ist damit die "psychische und physische Unversehrtheit gemeint « - durch »mangelnde Sensibilität« in den Asylunterkünften aufs »Spiel« gesetzt werde. Die Formulierung »aufs Spiel setzen« ist eine abgeschwächte Form der in der ersten Version des Artikels (»Hinterland«) verwendeten Formulierung »nicht gewährleistet wird «. »Aufs Spiel setzen« verweist darauf, dass die psychische und physische Unversehrtheit riskiert oder deren Verletzung vielleicht sogar leichtfertig in Kauf genommen wird. Der Begriff der Sensibilität, dessen Mangel den Asylunterkünften vorgeworfen wird, ist bereits im Diskursfragment von LesMigraS zum CSD häufiger verwendet worden. Sensibilität bedeutet »Empfindsamkeit « oder auch »Gespür für Verletzendes «. ${ }^{109}$ Es ist ein Begriff, der im Kontext von Critical-Whiteness-Diskursen für eine »Empfindsamkeit« oder ein »Gespür für Verletzendes«, das auf (Mehrfach-)Diskriminierung zurückgeht, steht. Critical Whiteness fordert insbesondere von sogenannten "privilegierten Personen« Sensibilität für (Mehrfach-)Diskriminierung. Eine durch mangelnde Sensibilität gekennzeichnete Unterbringungssituation sei nicht dazu in der Lage - hier wird festgeschrieben, was in der Unterkunft »eigentlich « möglich sein sollte -, die Geflüchteten, für die unter anderem das Asylverfahren eine »traumatische« und »belastende« Angelegenheit darstellt, zu entlasten. Der Gegensatz zwischen dem, was sein sollte - Entlastung aufgrund des belastenden Asylverfahrens -, und dem, was eigentlich ist - »zusätzliche Belastung« durch die Wohnsituation -, wird aufgemacht, um die Wohnsituation weiter als notwendigen Ort der Villa-Intervention festzuschreiben: Die schlechte Unterbringungssituation verstärke »die Tendenz sich aus Selbstschutz zurückzuziehen und mit niemandem über den eigentlichen Fluchtgrund zu reden $\ll$.

An diesen Satz anschließend folgt das Fazit: »So wird das Verfahren zu einem Glücksspiel.« Das »So« als Übergangswort verweist auf die Beziehung zwischen der Gefährdung in den Asylunterkünften und der Tatsache, dass das Asylverfahren zum »Glücksspiel« werde. Würde es nicht an Sensibilität in den Asylunterkünften fehlen, so suggeriert die Argumentation, wäre das Spiel nicht mehr ein derartiges »Glücksspiel«. Im anschließenden Satz wird das Glücksspiel allerdings auf die Wahrscheinlichkeit, an eine »verständnisvolle Rechtsberatung« oder an eine Person zu geraten, die sich

Duden: Sensibilität, https://www.duden.de/rechtschreibung/Sensibilitaet vom 24.11.2020. 
aufgrund »moralisch-religiöser Vorstellungen von Sexualität und Geschlechtsidentität « nicht für die »Geflüchteten« einsetzt, bezogen. Ähnlich wie im Kontext der Asylunterkunft wird hier die »Spiel«-Metapher aufgerufen. Während vorher auf das, was im Spiel riskiert wird, verwiesen wurde, ist es hier die »Willkürlichkeit« des »Spiels«. Damit wird das Asylverfahren im Gegensatz zu dem, was es eigentlich sein sollte - ein Ort der transparenten und gerechten Rechtsprechung -, als willkürlich und damit ungerecht kritisiert. Ein »Glücksspiel « ist aber auch ein »Spiel«, in dem das Casino, statistisch gesehen, letztlich immer gewinnt. In dieser Analogie wird Kritik an einem Asylverfahren geübt, in dem Geflüchtete nichts zu gewinnen haben, ihre Rechte folglich nicht gewährleistet werden. Ähnlich wie bereits durch die blutenden Hände wird auch hier Religiosität als Instanz, die tötet, festgeschrieben. Ob die Rechte von LGBTIQ-Asylsuchenden gewahrt werden, ist - so die zentrale Aussage des Abschnitts - ein »Glücksspiel«. Indem die Unterbringungssituation verbessert werde, könne zumindest die Wahrscheinlichkeit ein wenig erhöht werden, in diesem »Spiel« auch mal zu gewinnen. Alternative Asylunterkünfte seien notwendig, weil sich in diesem Bereich, im Gegensatz zu anderen Bereichen, noch nichts verbessert habe. Sie seien aber auch notwendig, weil sie im Asylverfahren entlastend - oder zumindest nicht zusätzlich belastend - sind und so die Chancen im »Glücksspiel« zumindest ein wenig verbessert werden könnten.

Im vierten Abschnitt wird - basierend auf Erfahrungen, von denen "Betroffene« berichten - erzählt, welchen verletzenden Situationen LGBTIQ-Geflüchtete ausgesetzt sind. Es werden aber auch die Überforderungen und »Abwehrreaktionen« der mehrheitsweißen Aktivist*innen reflektiert, die sich gegenüber den Erzählenden in einer Machtposition befinden.

Zunächst wird aufgezeigt, dass das, was die Behörden und Asylunterkünfte leichtfertig »aufs Spiel setzen « - das Leben oder zumindest die Unversehrtheit der Geflüchteten -, zu einer gelebten Realität für viele LGBTIQ-Geflüchtete wird:»Besonders schwierig wird es, wenn die Angst vor Übergriffen in den Unterkünften sich bewahrheitet und Mitasylwerbende zu Täter_innen werden. Psychische Häme, nicht glauben wollen, dass der Fluchtgrund der Wahrheit entspricht, bis hin zu Körperverletzungen und sexueller Gewalt, sind viel zu oft Erfahrungen, die [sic!] uns Betroffene erzählen.« Die Villa ist als Beratungs- und Unterstützungsort mit den Lebensrealitäten, die sich in die Körper der "LGBTIQ-Flüchtlinge« eingeschrieben haben und von denen sie sprechen, konfrontiert. Sie konstituieren sich aber nicht nur als Austausch- und Unterstützungsort, sondern auch als sich selbst reflektierendes Wir, das erkennt, dass es mit dieser Konfrontation überfordert ist und dass der »mehrheitsangehörige« Teil der Villa selbst auch oft nicht frei von Vorurteilen und Abwehrreaktionen ist. Selbstkritisch wird die eigene Machtposition und das eigene Selbstbild in Frage gestellt: »Die Macht zu haben, jemanden als glaubwürdig einzuteilen und die persönliche Abwehr von traumatischen Erzählungen, die das Selbstbild einer demokratischen und gerechten Gesellschaft in Europa stören.«In dieser Art und Weise, die eigenen Machtverhältnisse zu benennen und zu reflektieren, schließt die Villa abermals an Critical-Whiteness-Diskurse an. Die Villa konstituiert sich hier als eine Gemeinschaft, die mit den Lebensrealitäten der Geflüchteten konfrontiert ist, die aber auch reflektiert und die eigenen Machtpositionen, 
Vorurteile und Abwehrreaktionen, die in dieser Konfrontation zu Tage treten, erkennt und benennt.

Im fünften Abschnitt wird die Notwendigkeit betont, die »Unterbringung von LGBTIQGeflüchteten« zu einem zentralen Punkt des Villa-»Engagements « zu machen, damit positive Asylbescheide zugestellt werden können, die Unterbringung und Grundversorgung gewährleistet ist und eine zusätzliche Belastung zum Asylverfahren genommen wird.

In der ersten Version - dem »Hinterland «-Artikel - hat dieser Abschnitt noch die Überschrift »Wohnungen verbessern prekäre Lage«. Auch der erste Satz des Abschnitts wurde umformuliert. Im »Hinterland«-Artikel »wurde« die »sichere Unterbringung von LGBTIQ die geflüchtet sind, [...] zum Kernthema des Engagements«. In der Villa-BlogVersion »musste« sie zum »Kernthema« werden. Das »wurde«, als eine Beschreibung von etwas, dass stattgefunden hat, wird zu einem "werden müssen«, als eine Beschreibung von etwas, das notwendigerweise stattgefunden hat. Die Forderungen der »LGBTIQ-Flüchtlinge« nach Unterstützung, die Tatsache, dass Unterbringung ein wichtiger Ort der Unterstützungsmöglichkeit ist, und das emanzipatorische Wir legen die Notwendigkeit, die Unterbringung von Geflüchteten zum »Kernthema« des Villa-»Engagements« zu machen, nahe. Mit dem Begriff der »Prekarisierung « - mit dem die zusätzliche Belastung im Asylverfahren bezeichnet wird - wird auf einen wichtigen Begriff für solidarische Koalitionen in Judith Butlers Philosophie des Politischen angespielt. ${ }^{110}$ Prekarität - in dem Fall die schlechte Unterbringungssituation als zusätzliche Belastung zum Asylverfahren - wird zum Ausgangspunkt und zum verbindenden Moment des Villa-Engagements.

Im sechsten Abschnitt wird zunächst davon gesprochen, dass es für die Villa als Wohnprojekt auf der »Hand liegt«, sich neben der Aneignung von Beratungskompetenzen auch um Wohnungen für »LGBTIQ-Flüchtlinge« zu kümmern. Hierzu wurden Netzwerke genutzt und auch Verhandlungen mit der Politik geführt.

Zunächst einmal wird mit Bezug auf die Geschichte der Villa das »Engagement für sicheren und selbstbestimmten Wohnraum« von LGBTIQ Geflüchtetem als logische Fortführung/Konsequenz dessen, was die Villa ist - ein aus der Hausbesetzer*innenbewegung entstandenes Wohnprojekt - beschrieben (»[e]s liegt auf der Hand«). In den nächsten Absätzen wird aufgezählt, was die Villa im Namen dieses Ziels bereits erreicht hat und was gerade noch verhandelt wird: »es wurden in einem ersten Schritt Wohnungen angemietet «, »seit geraumer Zeit wohnt etwa ein pakistanischer Schwuler in der Hausgemeinschaft«, »[die Villa] unterstützt auch aktiv bei der Wohnungssuche«, »verstärkt [...] Kompetenzen [...] in der Rechtsberatung und [hat] Netzwerke zu Anwält_innen und Asyl-NGOs«, »[v]erhandelt [...] einen Sonderstatus für LGBTIQ Flüchtlinge« und »wirbt«»für 3 selbstverwaltete Wohnhäuser«. Die Villa konstituiert sich hier erstens als ein Wir, das bereits aktiv handelt, und zweitens als ein Wir, für das das »Engagement« für Räume, insbesondere Wohnräume, schon immer ein zentrales, zusammenhaltendes Element war. Die Notwendigkeit, sich für Wohnraum einzusetzen, 
wird erneut in Bezug auf die allgemeine Belastung im Asylverfahren begründet: »Denn die Isolation und Vereinsamung in den Standardunterbringungen ist für viele LGBTIQ ein weiteres Hindernis zu einem fairen Verfahren.«Um dieses Argument zu stützen, wird zusätzlich das Beispiel von Trans*-Frauen in den Asylunterkünften angeführt, die oft nicht einmal duschen können, weil »ihre Intimsphäre nicht geschützt wird«. Der Kampf um Wohnraum für LGBTIQ-Geflüchtete wird als wichtiger Kampf um Unterstützungsstrukturen ausgewiesen. ${ }^{111}$ Wohnraum wird als Möglichkeitsbedingung, das eigene Recht auf Asyl (faireres/gerechtes Asylverfahren) einfordern zu können, in den Villa-Politiken reklamiert. Die Villa konstituiert sich als ein Wir, das solidarisch mit denjenigen ist, die - mit Butler gesprochen - insofern "prekarisiert« sind, als sie aufgrund einer »ungleichen Verteilung von Gefährdetheit « mehr auf Unterstützungsmöglichkeiten angewiesen sind als andere. Die Einrichtung fordert gemeinsam mit den Geflüchteten ebendiese Unterstützungsmöglichkeiten ein.

Im siebten und letzten Abschnitt wird darauf verwiesen, dass solidarisches Handeln auch heiße, Orte zu schaffen, an denen Geflüchtete sich austauschen und vernetzen können. Solidarisches Handeln bedeute zudem, eine Politik zu verfolgen, in der die eigenen »Privilegien [...] im Sinne der Betroffenen« genutzt werden.

In diesem letzten Abschnitt grenzt sich die Villa noch einmal explizit von einer solidarischen Politik ab, die den »Geflüchteten« von »oben« hilft. Allerdings wird zumindest semantisch - die von Spivak kritisierte Geber*innen-und-Nehmer*innenDichotomie wiederholt, wenn es heißt, dass es darum gehe, den Geflüchteten »Raum zu geben, um ihr Wissen und ihre Erfahrung einzubringen«. Diese Wortwahl widerspricht aber den vorherigen Darstellungen des Artikels, denen zufolge zunächst die Geflüchteten Unterstützung von der Villa eingefordert haben. Die Aussage, dass solidarische Politik bedeute, dass Menschen mit »Privilegien« ihr politisches Handeln »nach den Bedürfnissen der Mehrheitsangehörigen« ausrichten, ist ebenfalls im Kontext der Critical Whiteness einzuordnen. Abschließend wird - ebenfalls Bezug nehmend auf CriticalWhiteness-Diskurse - betont, was in den Beschreibungen der vorherigen Abschnitte bereits deutlich wurde: Solidarische Politiken dürften keine Top-down-Politiken sein und es gehe darum, dass diejenigen, die weniger prekär leben, die eigenen Ressourcen nutzen, um diejenigen zu unterstützen, die mehr auf Unterstützung angewiesen sind. Damit wird die Vorgehensweise der Villa, die den eigenen Beschreibungen zufolge diesen Anforderungen zu entsprechen scheint, als solidarisch festgeschrieben.

\section{Prekarität als das verbindende Moment}

Die Villa stellt sich in der Auseinandersetzung mit Mehrfachdiskriminierung im Kontext von Flucht und Asyl als ein solidarisches Wir her. Dieses Wir ist insofern solidarisch, als die Villa ein »offener « Ort ist, an dem auch diejenigen, die noch nicht Teil der Villa sind, "gehört« werden. Die Villa ist auch solidarisch, weil die Einrichtung zunächst "zuhört " und nicht von oben herab entscheidet, wie andere unterstützt werden sollen. Das Villa-Wir ist aber auch insofern solidarisch, als dieses Zuhören reflektiert ist - die eigenen »Leerstellen« und »Widersprüche« werden kritisch in den Blick genommen - 
und trotz unbezahlter Arbeit die notwendige Verantwortung angenommen wird. Die Villa ist also zunächst in Bezug auf sich selbst solidarisch - indem sie "zuhört« und »reflektiert« - , sie ist aber auch solidarisch, indem sie Verantwortung übernimmt und handelt (die Berater*innen bilden sich fort, es werden Netzwerke geknüpft und Forderungen an die Stadt gestellt). Die Art und Weise, wie Verantwortung übernommen wird, begründet die Villa aus der eigenen Geschichte - Wohnen als ein Thema, das für die Villa auf der "Hand liegt« - , aber auch in Bezug auf das Asylverfahren (Wohnen als mögliche Be- oder Entlastung im Asylverfahren). Dabei ist Prekarität das verbindende Moment der solidarischen Villa-Politik, das auf mehreren Ebenen hergestellt wird. Auf der Bild-Ebene verweisen die blutenden Hände als Anklage an die ÖVP und die Kirche auf die gemeinsam geteilte Gefährdung. Während queere Menschen im Zuge der AidsKrise dem Tod ausgesetzt waren, sind mit Jasbir Puars Worten Geflüchtete die neuen death-figures. ${ }^{112}$ Zweitens wird eine verbindende Beziehung über den Begriff der Prekarität hergestellt, indem sich das, was die Villa aufgrund der eigenen Vergangenheit als Hausbesetzer*innenprojekt geworden ist, nahtlos einreiht in den Kampf gegen Prekarität - als Kampf um Wohnraum für queere Geflüchtete. Dass der Kampf um Wohnraum bevorzugter Ort der Interventionen gegen Prekarität ist, wird wiederum über dessen zentrale Rolle im Asylverfahren legitimiert, aber auch darüber, dass in diesem Bereich, der Villa zufolge, bisher wenige Fortschritte erreicht wurden.

\subsubsection{Das Lesbisch-schwule Stadtfest}

Im Analysezeitraum (2010 bis 2016) ist die Solidarisierung mit Gruppen mit Rassismuserfahrungen - neben den sehr dominanten Forderungen nach Räumen und finanziellen Ressourcen für das eigene Projekt - ein zentrales Thema in den Stellungnahmen von Lambda BB. So solidarisiert sich Lambda BB - wie bereits in der Analyse zur Pride herausgestellt wurde - in der Rede zur Entgegennahme des »Soul of Stonewall Award « mit Gruppen wie LesMigraS. In einer anderen Stellungnahme kritisiert die Einrichtung die Unsichtbarmachung von Queers of Color und Trans*-Personen im Film "Stonewall ${ }^{113}$ Im für die Feinanalyse ausgewählten Diskursfragment aus dem Jahr 2015 zeigt sich das Jugendnetzwerk Lambda BB (JNLBB) solidarisch mit der Nicht-Teilnahme von LesMigraS am Lesbisch-schwulen Stadtfest. Das Lesbisch-schwule Stadtfest ist, nach dem CSD, das zweitgrößte Community-Event in Berlin. Die Kritik am Lesbisch-schwulen Stadtfest - mit der das Jugendnetzwerk Lambda BB sich in der Stellungnahme solidarisiert - hat eine längere Vorgeschichte. Das Jugendnetzwerk Lambda BB ist (wie auch LesMigraS und TrIQ e.V.) Mitglied im »Netzwerk Diskriminierungsfreie Szene für alle ${ }^{114}$ Bereits 2012 hat das »Netzwerk Diskriminierungsfreie Szene für alle« den Ver-

112 J. K. Puar: Terrorist Assemblages, xxi.

113 Jugendnetzwerk Lambda:BB: Pressemitteilung Nr. 03-2015 ... zu Roland Emmerichs Film »Stonewall« 2015, https://www.lambda-bb.de/wp-content/uploads/2012/12/StellungnahmeStonewall.pdf vom 23.11.2020.

114 Das Netzwerk wurde 2010 in Berlin von »Cladt e.V.« gegründet und hat vor dem Hintergrund, dass eigene Diskriminierungserfahrungen nicht davor schützen, selbst zu diskriminieren, das Ziel, queere Räume«-insbesondere in Bezug auf Mehrfachdiskriminierung -»diskriminierungsärmer« werden zu lassen. Hierzu gibt es regelmäßig Netzwerktreffen, in denen Austausch stattfindet; zu- 
anstalter*innen (Regenbogenfonds der schwulen Wirte e.V.) mitgeteilt, dass es auf dem Stadtfest zu rassistischen und trans"-feindlichen Übergriffen gekommen sei. Dies wurde mit der Begründung, dass es keine belegbaren Vorfälle gäbe (da sie nicht bei Polizei oder Ordnungshüter*innen gemeldet wurden), wenig beachtet beziehungsweise als Antwort einzig ein »Diskriminierungstelefon« eingerichtet. Ein Jahr später veröffentlicht TrIQ gemeinsam mit »Gladt e.V.« eine Stellungnahme, in der berichtet wird, dass die Vorfälle sich wiederholt hätten. Es wird gefordert, dass die »Kritik und Forderungen von verschiedenen Organisationen [...] ernstgenommen werden «. ${ }^{115} 2015$ veröffentlicht LesMigraS eine politische Stellungnahme zur Nicht-Teilnahme am Lesbisch-schwulen Stadtfest. ${ }^{116}$ Als Grund nennt LesMigraS zum einen die Tatsache, dass die Organisator"innen bis dahin - mit Ausnahme der Einrichtung eines SOS-Telefons - nicht auf die vorkommenden Gewalt- und Diskriminierungsfälle reagiert hätten. Auslöser war zudem das Werbeplakat zum Stadtfest:

»Das Stadtfest wirbt mit dem Motto: `Cleiche Rechte für Ungleicheく, in diesem Jahr sowohl auf Deutsch wie auch auf Arabisch. Der arabische Satz ist spiegelverkehrt und nicht korrekt übersetzt. Die Zeichnung von zwei sich küssenden Frauen ist stereotypisierend und repräsentiert Lesben und/oder Muslima als Publikum des Festes, allerdings ohne dafür zu sorgen, dass diese keiner sexistischen und rassistischen Diskriminierung ausgesetzt sind. Wir kritisieren die Praxis, andere nach eigenen (stereotypen) Vorstellungen darzustellen, statt sie selbst zu Wort kommen zu lassen. « $^{117}$

Im Anschluss an diese Pressemitteilung kam es auf Social-Media-Plattformen (LesMigraS hat die Pressemitteilung beispielsweise auf der eigenen Facebook-Seite verlinkt) zu heftigen Diskussionen, auf die auch das Jugendnetzwerk Lambda BB in seiner Pressemitteilung verweist. Der Vorstand des Regenbogenfonds weist die Vorwürfe von LesMigraS noch im Juni des gleichen Jahres zurück und behauptet, das Plakat sei erfolgreich gewesen, da es ja Aufmerksamkeit bekommen habe. LesMigraS selbst nimmt im Jahr 2016 wieder am Lesbisch-schwulen Stadtfest teil, nachdem sich - nach eigener Aussage infolge der Auseinandersetzungen im Jahr 2015 einiges verändert habe. ${ }^{118}$

Die im Fokus der Analyse stehende Stellungnahme des Jugendnetzwerks Lambda BB schließt an die Stellungnahme von LesMigraS aus dem Jahr 2015 an, in der die Einrichtung die Nicht-Teilnahme verkündet. Der Titel der Stellungnahme von Lambda BB lautet entsprechend: »Stellungnahme zur Nicht-Teilnahme der Lesbenberatung/Les-

dem werden Veranstaltungen und Workshops organisiert. Netzwerk diskriminierungsfreie Szene für alle: Das Netzwerk, https://diskriminierungsfreieszenenfueralle.wordpress.com/netzwerke/ vom 24.11.2020.

115 TransInterQueer e.V./Cladt e.V.: Pressemitteilung von TransInterQueer e.V. 2013, https://www.trans interqueer.org/download/pm/PM_Stadtfest2013.pdf vom 24.11.2020.

116 LesMigraS: Stellungnahme zum Lesbisch-Schwulen Stadtfest 2015, https://lesmigras.de/tl_fil es/lesbenberatung-berlin/Newsletter_/Stellungnahme_2015_Stadtfest_Lesbenberatung.pdf vom 24.11.2020.

117 Ebd.

118 LesMigraS: Archiv. 
MigraS am Lesbisch-Schwulen Stadtfest 2015 «. ${ }^{119}$ Die Überschrift ist fast identisch mit der Überschrift der Pressemitteilung von LesMigraS, mit dem Unterschied, dass es dort heißt: "Stellungnahme der Lesbenberatung Berlin e.V. zur Nicht-Teilnahme am Lesbisch-Schwulen Stadtfest $2015 . \ll^{120}$ Formal sind - ähnlich, wie es auch in den Pressemitteilungen der anderen Projekte der Fall ist - die Pressemitteilungen des Jugendnetzwerks Lambda BB (fast) immer gleich gestaltet. Im Fall von Lambda BB befindet sich am Kopf, vor einem gelben Hintergrund mit pinken Streifen, ${ }^{121}$ oben rechts der Name und das Logo des Jugendnetzwerks Lambda:: Berlin-Brandenburg e.V. Die Pressemitteilung selbst ist zwei Seiten lang und in fünf Abschnitte unterteilt. In den letzten drei Abschnitten ist jeweils der erste Satz - die zentrale Aussage - fett gedruckt. Am Ende der Pressemitteilung befindet sich unten rechts die Anschrift des Jugendnetzwerks Lambda BB und erneut der Name und das Logo.

Im ersten Abschnitt erklärt sich das JNLBB solidarisch mit der Nicht-Teilnahme von LesMigraS und der Lesbenberatung am Lesbisch-schwulen Stadtfest 2015 und äußert sich beunruhigt über die Reaktionen auf den Social-Media-Plattformen. Lambda BB wolle, dass die Kritik von LesMigraS ernst genommen wird.

Es wird zunächst ganz grundsätzlich - wie es in einer Pressemitteilung üblich ist die zentrale Aussage der Pressemitteilung formuliert: Lambda BB solidarisiert sich mit LesMigraS. Nachdem in der Überschrift bereits deutlich wird, worum es geht - das in der Community aktuell kontrovers diskutierte Thema: die Nicht-Teilnahme von LesMigraS und der Lesbenberatung am Lesbisch-schwulen Stadtfest 2015-, wird im ersten Satz bereits klar, wie sich Lambda BB dazu positioniert: solidarisch. Im Folgenden wird ausgeführt, was eine solidarische Politik für Lambda BB bedeutet und was das verbindende Moment der Solidarisierung ist. Zunächst wird die Solidarisierung, als durch Affekte vermittelt, beschrieben. Die Reaktionen auf den Social-Media-Plattformen habe Lambda BB »erschrocken« und »beunruhigt« wahrgenommen. Hier werden Gefühle, die negativ berühren und sogar »erschrecken«, als verbindendes Moment aufgerufen. Die Social-Media-Diskussionen haben bei Lambda BB Angst (»erschrocken«) und Sorge (»beunruhigt«) ausgelöst. Es sind Affekte, die Teil unserer Offenheit gegenüber der Welt und damit auch gegenüber den anderen sind. Sie sind Butler zufolge der Stoff der Kritik, ${ }^{122}$ aber auch Stoff, der eine »ethische Begegnung « ${ }^{123}$ möglich macht. Diese Affekte werden als verbindendes Moment einer solidarischen Politik aufgerufen. Um diese negativen Affekte zu »beruhigen«, wünscht sich Lambda BB, »dass die Kritik, die hinter der Nicht-Teilnahme steht, gehört und ernst genommen wird.«

\footnotetext{
119 Jugendnetzwerk Lambda:BB: Stellungnahme zur Nicht-Teilnahme der Lesbenberatung/LesMigraS am Lesbisch-Schwulen Stadtfest 2015, https://www.lambda-bb.de/wp-content/uploads/2015/06/St ellungnahme_Nicht-Teilnahme-Lesbenberatung_FINAL_18062015.pdf vom 23.11.2020. LesMigraS: Stellungnahme zum Lesbisch-Schwulen Stadtfest 2015.

121 Das sind die gleichen Farben und Anordnungen wie auf der Homepage von Lambda BB.

122 J. Butler: Raster des Krieges, S. 40.

123 J. Butler: Anmerkungen zu einer performativen Theorie der Versammlung, S. 133.
} 
Im zweiten Abschnitt wird beschrieben, warum - nach dem Verständnis von Lambda BB - die Lesbenberatung und LesMigraS nicht am Lesbisch-schwulen Stadtfest teilnehmen. Die Organisator*innen des Stadtfests würden »rassistische, sexistische und transphobe Übergriffe« nicht anerkennen und entsprechend auch nichts dagegen unternehmen. Das diesjährige Plakat veranschauliche das gut.

In diesem Abschnitt versucht Lambda BB, die zentralen Gründe für die NichtTeilnahme von LesMigraS zu benennen, ohne diese Benennung als letztgültig wahre Aussage zu setzen. Stattdessen werden die genannten Gründe als »eigene« Interpretation markiert: »Nach unserem Verständnis liegen die Gründe dafür im Wesentlichen [...].« Damit positioniert sich Lambda BB als ein Wir, das eine gewisse Vorsicht walten lässt, wenn im Namen anderer Gruppen gesprochen wird. So wird implizit ein Gegensatz zum Lesbisch-schwulen Stadtfest hergestellt, das ohne Beteiligung der entsprechenden Gruppen ein Plakat, das ebendiese Gruppen repräsentieren soll, erstellt hat.

Im dritten Abschnitt wird zunächst fett hervorgehoben erklärt, dass die Kritik von LesMigraS auch als Kritik an allen, die am Stadtfest teilnehmen, verstanden werde. Die Organisator*innen hätten noch nicht genug getan, um »umfassende diskriminierungssensible Strategien « umzusetzen. Nicht alle könnten sich in der Community sicher fühlen, wobei die Kritik insofern auch eine »Chance« sei, als sie die Möglichkeit der Selbstkritik eröffnet. Entsprechend möchte das Jugendnetzwerk Lambda BB die Kritik von LesMigraS und die damit einhergehende Selbstkritik am eigenen Stand thematisieren, um Sichtbarkeit zu schaffen und Verantwortung zu übernehmen.

Lambda BB interpretiert im dritten Abschnitt die Kritik von LesMigraS als eine Kritik an allen, die am Stadtfest teilnehmen, und nicht nur an den Organisator*innen. Insofern, als Lambda BB also am Lesbisch-schwulen Stadtfest teilnimmt, fühle sich die Einrichtung durch die Kritik von LesMigraS auch angerufen: »Wir möchten diese Kritik - auch als Kritik an uns selbst - aufnehmen«. Indem die Einrichtung die Kritik von LesMigraS als Selbstkritik annimmt, konstituiert Lambda BB sich als Wir, das offen für Kritik ist. Lambda BB nimmt die Kritik auf eine bestimmte Art und Weise an, versteht sie als »Warnsignal und Chance«. Die Tatsache, dass sich in der Community »nicht alle sicher sich fühlen können«, sei ein »Warnsignal«. Der Ausdruck »Warnsignal« ist ein Kollektivsymbol, das auf eine akute Gefahr verweist. In diesem Fall ist die Community als »sicherer Ort für alle« in Gefahr. In einer Umkehrung legt die - durch das Bild des »Warnsignals« beschriebene - Bedrohung eine (utopische) Vorstellung einer Community nahe, in der sich alle sicher fühlen können. Sich mit LesMigraS zu solidarisieren, bedeutet für Lambda BB nicht, ebenfalls auf die Teilnahme am Lesbisch-schwulen Stadtfest zu verzichten, sondern stattdessen durch die Teilnahme auf die eigene Solidarität und Kritik aufmerksam zu machen: „Wir möchten diese Kritik - auch als Kritik an uns selbst - aufnehmen und zumindest an unserem Stand thematisieren.« Die solidarische Teilnahme wird mit der Notwendigkeit begründet, zu sensibilisieren und Ausschlüsse sichtbar zu machen: »Nur in einer Auseinandersetzung können wir versuchen, weiterhin Ausschlüsse sichtbar zu machen und verantwortungsvoll zu sensibilisieren. «Lambda BB konstituiert sich - indem es die Kritik von LesMigraS auch als Kritik an sich selbst versteht - in diesem Abschnitt als Wir, das selbstkritisch ist. Gleichzeitig stellt Lamb- 
da BB sich auch als verantwortungsvolles Wir her, das Sichtbarkeit schafft und in die Auseinandersetzung geht.

Im vierten Abschnitt wird noch einmal wiederholt, dass Lambda BB vor allem von den Reaktionen auf die Stellungnahme beunruhigt sei. Die »rassistischen, sexistischen, islamfeindlichen und/oder lesbenfeindlichen« Reaktionen würden auf die Notwendigkeit und Berechtigung der Kritik von LesMigraS verweisen. Sie würden auch zeigen, dass die Stellungnahme von LesMigraS nicht verstanden beziehungsweise nicht ernst genommen wurde. Diesen ignoranten Umgang lehnt Lambda BB ab.

Lambda BB betont - wie schon im ersten Abschnitt - die eigene "Beunruhigung « und das »Erschrecken« »insbesondere über die Reaktionen auf die Stellungnahme«. Die »Beunruhigung « und das »Erschrecken« beziehen sich auch hier auf die OnlineReaktionen. Nicht die Erfahrungen, von denen LesMigraS und andere Einrichtungen (seit Jahren) berichten, werden als Auslöser der »Beunruhigung und des Erschreckens« - damit als affektiv verbindendes Moment - genannt, sondern der Hatespeech, der online sichtbar ist: »Eine Großzahl der Kommentare, die online gemacht wurden, zeigen auf erschreckende Weise, wie notwendig und berechtigt die Kritik ist.« Dabei wird implizit die Logik des Lesbisch-schwulen Stadtfestes selbst wiederholt, nach der (nur) sichtbare - gemeldete - Übergriffe »von Gewicht sind«. Es sind die medial vermittelten und verlängerten Diskriminierungen und Übergriffe, die Affekte hervorrufen, die wiederum auf die Notwendigkeit solidarischer Politiken verweisen. An diesem Punkt scheint die Überschrift in die Irre zu leiten. Es ist nicht die Nicht-Teilnahme von LesMigraS, zu der hier Stellung bezogen wird, sondern es sind die darauffolgenden OnlineHass-Kommentare. Jenseits vom öffentlich sichtbaren Online-Hatespeech scheinen rassistische und trans* phobe Übergriffe für andere (mehrheitsweiße) teilnehmende Organisationen nicht derart sichtbar zu sein und damit weniger solidarisch verbindende Affekte hervorzurufen. Erst die durch die Online-Hassreden ausgelösten Affekte bestätigen die Kritik von LesMigraS. Indem Hassreden ins Zentrum des Lambda BB-Statements gestellt werden, wird aber auch auf eine (gemeinsam geteilte) Verletzlichkeit verwiesen. ${ }^{124}$ Es sind Affekte, ausgelöst durch sichtbare Verletzungen, die das verbindende Moment der Solidarisierung mit LesMigraS herstellen.

So wenig, wie Lambda BB davon spricht, auf welchen Plattformen die Diskussionen stattgefunden haben, so wenig wird in der folgenden Argumentation auch erklärt, warum und inwiefern die Kommentare »rassistisch, sexistisch, islamfeindlich und/oder lesbenfeindlich « sind. Ersteres ruft die Community in Berlin, die die Diskussionen kennt, als Zielgruppe der Pressemitteilung an. Letzteres wird als eindeutig und deshalb als nicht zu begründend festgeschrieben. Es ist aber etwas - so die Pressemitteilung weiter -, das sich so ähnlich vor kurzem im Zuge der Kritik von »Gladt e.V.« an der Kiss-in«-Aktion von »Maneo ${ }^{125}$ bereits gezeigt habe. Auch hier wird wieder die

124 J. Butler: Haß spricht, S. $26 f$.

125 Gladt e.V.: Stellungnahme CLADT e.V. zum Kiss In von Maneo am 17. Mai Internationaler Tag gegen Homophobie und Trans*phobie 2015, http://dkp-queer.de/stellungnahme-gladt-e-v-zumkiss-in-von-maneo-am-17-mai-internationaler-tag-gegen-homophobie-und-transphobie/9158 vom 24.11.2020. 
Berliner Community als Zielgruppe angesprochen, wenn ohne zusätzliche Erklärungen Community-interne Debatten aufgegriffen werden. Gleichzeitig bestätigt die Wiederholung des Falls die »Sorge« und »Angst« von Lambda BB. Die Online-Reaktionen werden abschließend als »ignoranter Umgang « bezeichnet, von dem sich Lambda BB mit einem Ausrufezeichen entschieden distanziert: »Wir lehnen diesen ignoranten Umgang mit der Stellungnahme entschieden $a b !$ L Lambda BB konstituiert sich in diesem Abschnitt als ein Wir, das als Teil der (mehrheitsweißen) Gemeinschaft aufgrund der durch Online-Hassreden ausgelösten Affekte (Sorge und Angst), im Gegensatz zum »ignoranten Umgang« der Anderen, die Kritik von LesMigraS ernst nimmt.

Im letzten Abschnitt wird die Überzeugung ausgesprochen, dass die »weiße, christliche, cis-männliche und nicht-behindert dominierte Community [...] auf Kritik angewiesen ist «. Die Online-Reaktionen würden zeigen, dass eine kritische Auseinandersetzung mit Diskriminierung in der Community noch immer kein Konsens sei. Lambda BB werde die Selbstkritik und die Nicht-Teilnahme von LesMigraS am diesjährigen Stadtfest am eigenen Stand thematisieren und sichtbar machen, um die Berliner Community als einen Ort gegenseitiger Kritik wiederherzustellen.

Zunächst positioniert Lambda BB sich und die Community als »weiße, christliche, cis-männliche und nicht-behindert dominiert«. Hier wird eine Praxis der »Selbstpositionierung «, die auf die Critical Whiteness Studies zurückgeht, bejaht. Mit dieser Positionierung gehe einher - zumindest setzt Lambda BB das im Schreiben als Selbstverständlichkeit voraus -, dass mensch »auf Kritik aus unterschiedlichen Bewegungen angewiesen« ist. Es wird ein Zusammenhang zwischen der Positionierung und der Angewiesenheit auf Kritik hergestellt. Lambda BB verweist im Anschluss auf eine Diskrepanz zwischen dem mit der Positionierung einhergehenden Soll-Zustand und dem Status quo: »die Internetreaktionen zeigen, dass die Sichtbarmachung von Diskriminierung nicht als der gemeinsame und notwendige Lernprozess verstanden wird, der er sein sollte.« Hier wird ein Verfahren der Kritik, das bereits in vielen Diskursfragmenten zur Pride verwendet wurde, aufgenommen. Es wird eine Diskrepanz zwischen dem, was die Community eigentlich sein sollte - offen für Kritik - , und dem, was sie aktuell ist, offengelegt. Im Gegensatz zu dem, was von der Community im Namen dessen, was sie sein soll, gefordert wird, stellt Lambda BB sich als ein Wir her, das dieses Soll bereits erreicht hat: »Unsere Teilnahme versucht, verantwortungsvoll zu sein und unsere eigenen Versäumnisse und Möglichkeiten in den verschiedenen Debatten zu reflektieren.« Dabei spricht Lambda BB explizit nicht für LesMigraS, sondern für die mehrheitlich weiße Community, für die »das Fehlen der Lesbenberatung« auch ein Problem darstelle. Lambda BB positioniert sich auch hier in Abgrenzung zum Lesbisch-schwulen Stadtfest, das eine Gruppe repräsentiert, die es nicht repräsentiert. Im Gegensatz dazu erhebt das Jugendnetzwerk Lambda BB nicht den Anspruch, für LesMigraS zu sprechen. Das Jugendnetzwerk spricht stattdessen als »Teil einer mehrheitlich weißen Community« und fordert in diesem Sprechen eine Community, die offen ist für Kritik. Denn das sei es, was die Berliner Community ausmache: "Gerade in Berlin sind die verschiedenen LGBTI*Bewegungen das, was sie sind, weil sie sich kontinuierlich gegenseitig kritisieren und ernst nehmen! Lambda BB fordert eine Community, die sich wieder auf das zurückbesinnt, was sie Lambda BB zufolge eigentlich ist, was sie ausmacht, was sie »besonders« 
macht. Hier wird im Namen einer lokalen Sentimentalität auf das »Exklusive« als das, was die Berliner Community eigentlich ist, verwiesen. Das, was in Gefahr ist - jene Bedrohung, die affektiv aufgerufen wird - , ist die Community als das, was sie ist. Das verbindende Band solidarischer Politiken ist der Wunsch, die Community als das, was sie ausmacht - als Ort der kollektiven (wertschätzenden und ernst nehmenden) Auseinandersetzung - , aufrechtzuerhalten. Lambda BB konstituiert sich als ein Wir, das sich um die Community sorgt, das Angst hat, dass die Community das verliert, was sie eigentlich ist und ausmacht: eine Gemeinschaft, die insofern solidarisch ist, als sie offen ist für Kritik und diese auch ernst nimmt.

Das Jugendnetzwerk Lambda BB stellt sich als ein solidarisches Wir her, das insofern solidarisch ist, als es Solidarität von den anderen fordert und im Gegensatz zu den anderen offen ist für Selbstkritik. Die verbindenden Elemente der Solidarisierung sind die durch Online-Hassreden ausgelösten Affekte der Sorge und der Angst. Es sind auf der einen Seite Affekte, die auf sichtbare Verletzungen zurückgehen und somit eine »ethische Bindung« an die »Gefährdeten« begründen. Auf der anderen Seite sind es Affekte, die aus einer Sorge und Beunruhigung darüber resultieren, dass das, was die Community »besonders« macht, in Gefahr ist: eine offene und damit solidarische Community.

\section{Solidarität als Selbstkritik und Forderung}

Im Umgang mit Rassismus konstituieren sich sowohl die Villa als auch das Jugendnetzwerk Lambda BB als solidarische, politische Gemeinschaften. Was ist das Verbindende dieser solidarischen Politiken? Und wie tritt dieses verbindende Moment in Erscheinung? Bei der Villa ist Prekarität - also die ungleiche Verteilung von Gefährdetheit - das Band, das solidarische Koalitionen zusammenhält. Das gemeinsame Band im Kampf gegen die ungleiche Verteilung von Ressourcen - in dem Fall Wohnraum - wird in doppelter Hinsicht an die Geschichte der Villa geknüpft. Zum einen knüpft der Einsatz für die adäquate Unterbringung queerer Geflüchteter vermeintlich lückenlos an die Geschichte der Villa, die ihren Anfang in der Hausbesetzer*innenbewegung genommen hat, an. Zum anderen wird eine Verbindung geknüpft zwischen der Prekarität der Körper, die in der Aids-Krise mit dem Tod konfrontiert werden, und der Prekarität der Geflüchteten. Nicht nur Prekarität, als eine erhöhte Wahrscheinlichkeit zu sterben, sondern auch der »Feind« ist ein gemeinsam geteilter: die ÖVP und die Kirche. Es ist die kohärente Geschichte und die geteilte Erfahrung für der ÖVP und der Kirche als nicht betrauberbare Leben zu gelten, die Prekarität als verbindendes Moment der solidarischen Villa-Politiken aufruft. Beim Jugendnetzwerk Lambda BB sind es eher Affekte der Beunruhigung, Sorge und Angst, in denen sich Solidarität begründet. Insofern diese Affekte durch Verletzungen ausgelöst sind, ist es auch hier eine gemeinsam geteilte Verletzlichkeit, die Solidarität begründet. Viel mehr als das ist es aber die Sorge und Angst um die Community, die als das bedroht ist, was sie eigentlich ist und was sie so besonders macht: eine offene, selbstkritische Community. Einmal ist es Prekarität als gemeinsam geteilte Erfahrung, und einmal ist es die »Angst « um den Verlust einer offenen und selbstkritischen Community, die das Band solidarischer Politiken knüpfen.

Die Solidarität der beiden Projekte unterscheidet sich auch in Bezug auf die Art und Weise, wie die Notwendigkeit einer Solidarisierung in Erscheinung tritt. In den 
Erzählungen der Villa tritt die Notwendigkeit der Solidarisierung in Erscheinung, wenn und weil die Geflüchteten selbst die Villa als Ort der Unterstützung aufsuchen und in diesem Aufsuchen die Unterstützung, die von der Villa bisher noch nicht mitgedacht wurde, fordern. Indem die Villa "zuhört«, erkennt die Einrichtung die eigenen Leerstellen und die Notwendigkeit, Verantwortung zu übernehmen. Beim Jugendnetzwerk Lambda BB treten die Affekte, die auf die Notwendigkeit solidarischer Politiken verweisen, medial vermittelt auf. In den Sozialen Medien werden auch für das JNLBB die Rassismen und Verletzungen, von denen LesMigraS spricht, sichtbar. Die Notwendigkeit einer solidarischen Politik tritt nicht primär durch eine Praxis des »Zuhörens« in Erscheinung, sondern dadurch, dass Lambda BB die Verletzungen selbst live miterlebt. Für das JNLBB wird die Notwendigkeit einer solidarischen Politik vor allem durch die sich in »öffentlich sichtbare« Räume einschreibenden Hassreden sichtbar. Auch wenn sich die Art und Weise, wie die Notwendigkeit solidarischer Politiken in Erscheinung tritt und wie das Band einer verantwortungsvollen Bindung an die anderen geknüpft ist, unterscheidet, so ist doch beiden Projekten gemein, dass Solidarität auf der einen Seite bedeutet, selbstkritisch und offen zu sein, und andererseits bedeutet, aktiv Verantwortung zu übernehmen. Die Art und Weise, wie die jeweiligen Projekte solidarisch handeln, steht in Beziehung zu dem gemeinsamen Band der Solidarität. Die Villa setzt sich aktiv gegen die prekäre Situation der Geflüchteten ein. Lambda BB thematisiert die Selbstkritik und die von LesMigraS erfahrenen Verletzungen am eigenen Stand und fordert gleichzeitig eine selbstkritischere Community. Während solidarisch handeln für die Villa heißt, etwas gegen prekäre Verhältnisse zu unternehmen, heißt es für Lambda $B B$, sich für eine offenere, zuhörende Community einzusetzen und gleichzeitig selbst Offenheit zu zeigen.

\subsubsection{Es gibt verschiedene Arten, solidarisch zu sein}

Auch TrIQ fordert - wie schon in der Analyse zur Pride gezeigt wurde - insbesondere von der Community Solidarität mit Menschen, die Mehrfachdiskriminierung erfahren. Allerdings liegt, entsprechend des Arbeitsschwerpunktes, der Fokus hier klar auf Mehrfachdiskriminierung in Bezug auf Trans" und Inter*. TrIQ thematisiert aber auch Rassismus. Die Einrichtung ist, wie auch LesMigraS und Lambda BB, Mitglied des Netzwerks "Diskriminierungsfreie Szene für alle« und in weiteren kooperativen Projekten gegen Rassismus engagiert. ${ }^{126}$ In den von TrIQ produzierten Informations- und Aufklärungsbroschüren wird oft auch eine intersektionale Perspektive eingenommen und die mit »Gladt e.V.« gemeinsam verfasste Stellungnahme zum Lesbisch-schwulen Stadtfest 2013 verweist auf eine enge Zusammenarbeit von TrIQ mit antirassistisch arbeitenden queeren Einrichtungen. ${ }^{127}$ Exemplarisch für die Art und Weise, wie Rassismus

126 Cemeinsam mit »Cladt e.V.« hat TrIQ e.V. 2013 eine Pressemitteilung zu Diskriminierungsfällen am Lesbisch-schwulen Stadtfest veröffentlicht, und im Kontext des »Netzwerks Trans*-Inter*Sektionalität«, das von TrIQ e.V. gegründet und koordiniert wurde, wurden Plakate und Broschüren produziert, die explizit auch Rassismus thematisieren. 
bei TrIQ verhandelt wird, ist der auf der Homepage des »Gunda-Werner-Instituts« veröffentlichte Artikel »Danke für nix - Wie geht Christopher Street Day in solidarischen Communities? «. ${ }^{128}$

$\mathrm{Zu}$ Beginn dieses Artikels nimmt TrIQ Bezug auf das bereits in der Überschrift anklingende Motto des CSD - »Danke für nix« - , um eine Verbindung zwischen der Einrichtung und dem CSD herzustellen. Der CSD und TrIQ teilen eine Kritik an der Untätigkeit der Politik in Bezug auf ihre Interessen. Auch für Trans*-Personen sei es in den letzten Jahren politisch nicht wesentlich vorangegangen. Im Anschluss an das im ersten Abschnitt angerufene, mit der Community verbindende Moment - »Danke für nix « - findet ein inhaltlicher Bruch in der Erzählung statt. Der Bruch symbolisiert, dass es darüber hinaus nichts Gemeinsames zu nennen gibt. Der Bruch im inhaltlichen Aufbau der Argumentation spiegelt einen Bruch in der Beziehung zwischen TrIQ und der Community wider. Der (neue) Ausgangspunkt dieser Bruch-Geschichte - der Geschichte des Bruchs (mit der Community) - ist ebenfalls ein aktuelles Ereignis: das Hassverbrechen in Orlando, »bei dem 49 Menschen, vor allem aus queeren Latinx-Communities, getötet wurden $« .{ }^{129}$ Ausgehend von diesem Ereignis kritisiert TrIQ zunächst die rassistischen Praktiken der medialen Berichterstattung: Zum einen würden Sichtbarkeiten hergestellt, indem über bestimmte Ereignisse berichtet wird und über andere nicht. Zum anderen würden die Medien die Welt in eine "gute« westliche und eine »schlechte» nicht-westliche Welt einteilen: »Das Bild, das uns im Anschluss an dieses Hassverbrechen und weitere Anschläge verkauft wurde, teile die Welt, so die Kritik, wieder einmal in >gut < und >böse< ein. « Verschwiegen werde dabei, dass auch im »sogenannten « Westen »Rassismus, Transfeindlichkeit, Interfeindlichkeit und Homofeindlichkeit tötet«. TrIQ kritisiert, auf postkoloniale Perspektiven Bezug nehmend, eine rassistische mediale Praxis, die eine Dichotomie zwischen »dem Westen« und den »Anderen« herstellt.

Die zunächst gegenüber der medialen Berichterstattung geäußerte Kritik - und hier kommt der Bruch - trifft auch auf große Teile der Community zu: »Von diesen Zuschreibungen sind auch Menschen und Institutionen unserer Communities nicht frei - wenn wir uns an Aussagen gerade auch in den letzten Wochen erinnern. « ${ }^{130}$ TrIQ bricht mit den rassistischen Aussagen aus der Community, »an die wir uns erinnern«, und »ädt « dementgegen »dazu ein«, sich »antimuslimischem Rassismus, anderen Rassismen und Antisemitismus entgegenzustellen«. Es ist eine Einladung zu einer Feier, die einen Preis hat: »anecken, unbequem sein, keine einfachen Antworten haben«. Es ist aber auch eine Einladung an Gäste, die den Medien nicht auf den Leim gehen: »[sich] kein einfaches Bild verkaufen zu lassen«. Die Community wird als besserwissend angerufen: Sie weiß, dass auch im Westen »Transfeindlichkeit, Interfeindlichkeit und Homofeindlichkeit tötet«. TrIQ bricht mit dem CSD - der sich Rassismen nicht

128 TransInterQueer e.V.: » Danke für nix<-Wie geht Christopher Street Day in solidarischen Communities?«, in: Gunda Werner Institut, https://www.gwi-boell.de/de/2016/07/28/danke-fuer-nix-wie-g eht-christopher-street-day-solidarischen-communities vom 24.11.2020. Am 12.06.2016 wurden in einem Club in Orlando 49 Menschen getötet und 53 verletzt. Hier wird auf Diskussionen verwiesen, die »alle zu kennen«scheinen. An welche Aussagen da »erinnert« werden soll, bleibt unbenannt und scheint ein in der »Community « geteiltes Wissen zu sein, das vielleicht auch nicht nach außen getragen werden soll. 
entgegenstellt -, ruft aber gleichzeitig ein gemeinsames (besseres) Wissen an, das ein gemeinsames Band im Kampf gegen Rassismus zu knüpfen vermag. Dabei wird der Community implizit vorgeworfen, wider besseres Wissen Rassismen zu produzieren. Das gemeinsam geteilte Wissen lasse aber keine Ausreden zu. Die Community wisse, wie es - entgegen medialer Berichterstattung - wirklich sei, weshalb sie, so die Anrufung, die Verpflichtung habe, gegen Rassismen anzugehen. Es wird auf ein gemeinsam geteiltes Wissen verwiesen, um die Community an ihre Verpflichtung zu binden, solidarisch und eben nicht rassistisch zu sein.

Im weiteren Text wird bestimmt, was mit der Solidarität, die von der Community gefordert wird, gemeint ist. In einer solidarischen Community seien »mehr Menschen sichtbar und hörbar", es könnten alle »andocken«, auch die Trans*-Sexarbeiter*in und die geflüchtete oder arbeitslose Person. Die von TrIQ ausgewählten Beispiele, die veranschaulichen sollen, wie eine solidarische Community aussehen könnte, nehmen Bezug auf Zielgruppen und Themen, die TrIQ selbst im Rahmen der eigenen Arbeit fokussiert (Trans*-Sexarbeit, Arbeitslosigkeit, Flucht). Ähnlich wie das Jugendnetzwerk Lambda BB nutzt auch TrIQ die Forderung an die »unsolidarische Community«, um sich (implizit) selbst, in Abgrenzung zur Community, als solidarisch herzustellen. Während Lambda $B B$ sich als solidarisch konstituiert, indem die Einrichtung davon berichtet, was sie tut: die Abwesenheit von LesMigraS und die eigene Betroffenheit thematisieren, stellt TrIQ sich implizit als solidarisch her, indem das, was TrIQ in der eigenen Arbeit leistet, zum Vorbild für solidarisches Handeln wird. Während beim Jugendnetzwerk Lambda BB die Fähigkeit zu Selbstkritik der zentrale Gradmesser des solidarischen Miteinanders in der Community ist, bedeutet Solidarität bei TrIQ, dass »mehr Menschen sichtbar und hörbar sind«.

TrIQ fordert also im Sprechen über Rassismus eine solidarische Community, in der mehr Menschen sichtbar/hörbar sind, und stellt sich selbst, in Abgrenzung zu den Angerufenen, als solidarische Gemeinschaft her. Während Lambda BB Solidarität von der Community im Namen dessen fordert, was die Community zu etwas Besonderem macht - dass sie ein Ort kritischer Auseinandersetzung ist -, fordert TrIQ eine solidarische Community im Namen eines gemeinsam geteilten, besseren Wissens - demzufolge eine Einteilung zwischen dem Westen als "gut« und dem Rest als »schlecht« $\mathrm{zu}$ einfach ist.

Bei LesMigraS sind Mehrfachdiskriminierung und Rassismus in (fast) jedem produzierten Text zentral. Auch anlässlich des Internationalen Tags gegen Homophobie und Trans*diskriminierung 2015 - für dessen Themenspektrum LesMigraS eine Erweiterung um Inter" -Diskriminierung fordert, die LesMigraS selbst umsetzt - , formuliert LesMigraS ein Statement gegen Mehrfachdiskriminierung. ${ }^{131}$ Im Fokus der Pressemitteilung von LesMigraS steht die Forderung, insbesondere Trans* of Color in den Mittelpunkt der Arbeit zu stellen. Begründet wird diese Forderung zunächst über ein ähnliches Verfahren, wie es bereits bei TrIQ im Redebeitrag zum CSD zur Anwendung gekommen

131 LesMigraS: Pressemitteilung der Lesbenberatung e.V. Berlin zum Internationalen Tag gegen Homophobie und Trans*diskriminierung 17. Mai 2015, https://lesmigras.de/tl_files/lesmigras/pressem itteilungen/PM_IDAHOT_2015_LB.pdf vom 24.11.2020. 
ist. Der Tatsache, dass Homosexualität bereits entpathologisiert und entkriminalisiert wurde, folge kausal nun die Aufgabe, Trans*-Personen - und im Falle von LesMigraS speziell Trans* of Color - in das Zentrum der politischen Forderungen zu stellen. Die Relevanz der Forderungen von Trans*-Personen wird im Vergleich zu dem, was Homosexuelle bereits erreicht haben, festgeschrieben. Indem im Folgenden beispielhaft Diskriminierungs- und Gewalterfahrungen von Trans* of Color - insbesondere von geflüchteten Trans* of Color - angeführt und als Formen struktureller Diskriminierung ausgewiesen werden, wird Solidarität mit diesen Gruppen als Notwendigkeit gesetzt. Die Forderungen nach einer solidarischen Community würden einerseits auf die Notwendigkeit von Zufluchtsorten für Trans* of Color antworten: "Die so produzierten Ausschlüsse von Trans* of Color und ihrer Perspektiven erschwert es ihnen, die LSBTICommunities und Communities of Color als Schutz- bzw. Entfaltungsräume, frei von Diskriminierung, zu nutzen." Andererseits würden sie auch auf die Notwendigkeit, gemeinsam gegen strukturelle Diskriminierung anzugehen, antworten: »Rassismus, Homo- und Trans*diskriminierungen sind keine alleinigen Angelegenheiten der jeweils Betroffenen, sondern Diskriminierungsverhältnisse, die gemeinschaftlich und auf gesamtgesellschaftlicher Ebene vorausschauend bekämpft werden müssen.« Dabei führt LesMigraS bereits aus, was solche gemeinsamen Forderungen - die als Notwendigkeit gesetzt werden - beinhalten könnten: »Anerkennung von LSBTI als besonders schutzbedürftige Geflüchtete«, »diskriminierungssensible Unterbringung«, »mehrdimensionale Herangehensweise gegen Gewalt und Diskriminierung generell«, "Aufhebung des Diagnoseschlüssels und der pathologisierenden Stigmatisierung « von Trans* - und Inter*-Personen und "gesetzlich verankerte Antidiskriminierungs- und Antirassismustrainings«.

Ähnlich wie bei TrIQ und dem Jugendnetzwerk Lambda kreist auch bei LesMigraS das Thematisieren von Mehrfachdiskriminierung und Rassismus zentral um die Forderung nach einer solidarischen Community. Während Lambda BB eine solidarische Community im Namen dessen, was sie als selbstkritische Community zu etwas Besonderem besonders macht, einfordert, verlangt TrIQ eine solidarische Community im Namen eines gemeinsam geteilten Wissens als einen Ort, an dem mehr Menschen sichtbar und hörbar sind. LesMigraS fordert eine solidarische Community, die gleichsam Zufluchtsort und Ausgangspunkt für gemeinsame Kämpfe gegen strukturelle Diskriminierung ist. Während die Community als Zufluchtsort zugleich Grund und Ziel der Forderungen nach einer solidarischen Community ist, wird die solidarische Community als Ort gemeinsamer Kämpfe gegen strukturelle Bedingungen, im Vergleich zu dem, was Homosexuelle bereits erreicht haben, gefordert. Ähnlich wie Lambda BB und TrIQ konstituiert sich auch LesMigraS in den die Pressemitteilung abschließenden Worten als Gruppe, die bereits das tut, was von der Community im Namen der Solidarität gefordert wird: "Seit 30 Jahren [...] arbeitet LesMigraS [...] daran Rassismus, Homophobie und Trans*diskriminierung zusammenzudenken«. LesMigraS ist bereits seit drei Jahrzehnten der (solidarische) Ort, der von der Community eingefordert wird.

Auch die Milchjugend spricht über Rassismus. Die Thematisierungen sind jedoch weniger zentral als bei den Berliner Projekten und der Villa. In Ausgabe fünf des »Milch- 
büechli« aus dem Jahr 2013 wird in dem Artikel »Weiss ${ }^{132}$ ist eine Hautfarbe ${ }^{133}$ Rassismus und "Kritisches Weiss sein« thematisiert. Mit dem Themenschwerpunkt der Ausgabe »an der Oberfläche« ist laut Editorial zum einen die »Oberfläche« der Zeitung, auf der sich die Artikel und Fotos einschreiben, selbst gemeint, zum anderen aber auch, dass in den Artikeln des »Milchbüechli« mehr »Praxis« (Oberfläche) und weniger »Theorie« (Tiefe) im Vordergrund steht. Der Artikel »Weiss ist eine Hautfarbe» nimmt ebenfalls auf die »Oberfläche« Bezug: »Jede Oberfläche hat eine Farbe. Ist dir eigentlich schon einmal aufgefallen, dass du eine Hautfarbe hast?«. Der graphische Aufbau der Überschrift verweist bereits implizit auf eine zentrale Aussage des Artikels. So sind die weißen Buchstaben der Überschrift »Weiss ist eine Hautfarbe« nur aufgrund des schwarzen Balkens, der einen Kontrast ermöglicht, lesbar. Damit wird schon durch die graphische Darstellung der Überschriften Weiß als unsichtbare Norm und Schwarz als Kontrast dazu sichtbar. Der Artikel wurde von dem damaligen Chefredakteur und Mitbegründer des »Milchbüechli« verfasst und beginnt mit einer persönlichen Erfahrung, die auch Anlass für den Artikel war: nämlich seine Reise nach New York/Harlem, auf der ihm das erste Mal bewusst wurde, dass er weiß ist. Es wird also über eine konkrete, beinahe banal wirkende Erkenntnis/Erfahrung des Autors in das Thema eingeführt. Diese niederschwellige Einführung, die von einer beinahe einfachen Erfahrung ausgeht, bietet auch jenen (jungen) Leser*innen, die sich bis jetzt noch nicht intensiv mit Rassismus und antirassistischen Theorien auseinandergesetzt haben, eine Identifikationsmöglichkeit an. Diese Herangehensweise steht im Kontrast zu den Pressemitteilungen und Redebeiträgen den Berliner Einrichtungen, die mit Begriffen wie »entwicklungspolitische Äußerungen«, Einteilung der Welt in »gut« und schlecht» oder »Privilegien« bereits eine Auseinandersetzung mit intersektionalen, postkolonialen und Critical-Whiteness-Perspektiven voraussetzen. Der »Milchbüechli«-Artikel versucht, vergleichsweise niederschwellig in das Thema einzuführen, und bleibt insofern, wie bereits im Editorial angekündigt, an der »Oberfläche«.

Die Erfahrung, weiß zu sein, wird im Verlauf des Artikels eingeordnet. Indem ein Band zwischen den Diskriminierungserfahrungen der "falschsexuellen« Jugendlichen und den Rassismuserfahrungen geknüpft wird, versucht der Artikel zunächst einmal verständlich zu machen, was Rassismus bedeutet. Diskriminierungserfahrung als gemeinsam geteilte Erfahrung aufzurufen, ist ein Versuch, Nähe zwischen der »falschsexuellen« Gemeinschaft und Menschen, die Rassismus erfahren, herzustellen. Im Namen dieses Bandes wird Kritik an "falschsexuellen« Gemeinschaften geübt, die Rassismus nicht ernst nehmen: »Plötzlich anerkennen wir die Vielfalt Schwarzer Menschen nicht mehr. Diese Vielfalt, die wir für uns selbst immer einfordern. Der schwule Schwarze ist immer gleich. Ein Klischee. «Im Sinne des Kant'schen Kategorischen Imperativs - »Handle so, dass die Maxime deines Willens jederzeit zugleich als Prinzip einer allgemeinen Gesetzgebung gelten könne« - wird den Jugendlichen nahegelegt, gleichermaßen die Vielfalt Schwarzer Menschen anzuerkennen, wie für die eigene Vielfalt Anerkennung gefordert wird. Im Gegensatz zu einem rassistischen Verhalten wird 
im Namen einer gemeinsam geteilten Diskriminierungserfahrung ein kritisches Weißsein eingefordert: »Wir haben alle die Möglichkeit, kritisch weiss zu sein. Der Anfang dazu ist, eigenes, vielleicht unbewusst rassistisches Verhalten zu ändern. Klarheit darüber zu bekommen, dass meine Interpretation und Weltsicht(en) weisse sind. Meine Privilegien werden damit >demaskiert « und aus der Unsichtbarkeit gehoben.« Die eigene Arbeit an sich selbst - der kritische Umgang mit dem Weißsein - sei die notwendige Voraussetzung, letztendlich "gemeinsam dem Rassismus und der Homophobie entgegenzutreten«.

Anders als es in den Argumentationen bisher der Fall war, wird die kritische Auseinandersetzung mit Rassismus nicht im Namen einer exklusiven, weil offenen Community (Lambda BB), nicht im Namen einer (historisch betrachtet) gemeinsam geteilten Prekarität (Villa), nicht im Namen eines gemeinsam geteilten besseren Wissens (TrIQ), sondern im Namen einer gemeinsam geteilten Diskriminierungserfahrung angerufen. Im Gegensatz zu den meisten anderen Projekten verwendet die Milchjugend nicht den Begriff der Solidarität, um Rassismus zu thematisieren. Sie fordert von den anderen auch nicht einen anderen (solidarischen, selbstkritischeren) Umgang mit Rassismus. Stattdessen schafft die Milchjugend, ausgehend von der eigenen Erfahrung, Räume, um an der »Oberfläche« über Rassismus nachzudenken, und fordert dazu auf, den eigenen Rassismus zu reflektieren. Ohne dass der Begriff »solidarisch« fällt, ist Selbstkritik, wie beispielsweise Lambda BB sich als solidarisch herstellt, auch zentral in der Auseinandersetzung der Milchjugend mit Rassismus. So konstituiert sich auch die Milchjugend selbst als selbstkritisch im Umgang mit Rassismus. Ähnlich wie bei LesMigraS - mit dem Unterschied, dass LesMigraS die Forderungen an die Community stellt - wird ein Umgang mit Rassismus gefordert, indem mit dem Rassismus in der eigenen Gemeinschaft aufgeräumt wird und gemeinsam dem strukturellen Rassismus entgegengetreten wird. Letzteres bleibt bei der Milchjugend allerdings ein vager Ausblick in die Zukunft.

Grundsätzlich ist allen fünf Projekten gemeinsam, dass sie sich mit Rassismus und Mehrfachdiskriminierung auseinandersetzen und sich in Bezug auf Rassismus selbst auf jeweils spezifische Art als solidarische, politische Subjekte konstituieren. Eine Ausnahme stellt die Zeitschrift "Out! « des Landesverbandes des Jugendnetzwerks Lambda dar. Dort ist Mehrfachdiskriminierung vor allem an der Schnittstelle von LGBTIQ* und Behinderung ein wichtiges Thema. Auseinandersetzungen mit Rassismus sind im Analyse-Zeitraum wenig zentral beziehungsweise finden fast gar nicht statt. Es fällt aber umgekehrt, vor allem in der Rubrik »Denkwürdig« oder »Queergeblickt «, ${ }^{134}$ auf, dass Lambda im Schreiben die eigene westliche Welt in Abgrenzung zu der Intoleranz der Länder im globalen Süden und den Ländern der (ehemaligen) Sowjetunion (sowie der Katholischen Kirche) herstellt. Überschriften, wie sie beispielsweise in der Rubrik "Denkwürdig« in der Ausgabe $18^{135}$ aus dem Jahr $2011 \mathrm{zu}$ finden sind, sind repräsentativ für das, was in dieser Rubrik im Analysezeitraum dargestellt wird. Auf der einen Seite wird über die Intoleranz der Anderen berichtet,

134 Jeweils am Anfang der Zeitschrift gibt es in der Rubrik»Denkwürdiges/Queergeblickt«kurze Nachrichten die neusten »queeren « News betreffend.

135 Jugendnetzwerk Lambda: »Denkwürdig«, in: Internationalität, Out! 2011. 
wie folgende Überschriften der Ausgabe 18 zeigen: »LGBT IN ASERBAIDSCHAN UNERWÜNSCHT, ANTI-HOMOSEXUALITÄTS-GESETZ VORLÄUFIG VOM TISCH«. Auf der anderen Seite wird von toleranten Errungenschaften in der westlichen Welt berichtet: »NBA-CLUB HAT SCHWULEN PRÄSIDENTEN«; »GLEICHES RECHT FÜR LEBENSPARTNERSCHAFT«. Dabei werden positive wie negative Identifikationsmöglichkeiten hergestellt. Die negativen Identifikationsangebote betreffen immer Beispiele aus der nicht-westlichen Welt oder aus der Katholischen Kirche. So wird in der Kurznachricht »ANTI-HOMOSEXUALITÄTS-GESETZ VORLÄUFIG VOM TISCH« über einen »von christlichen Fundamentalisten initiierte[n]) Gesetzesentwurf zu einem Anti-Homosexualitätsgesetz, das auf der Tagesordnung des ugandischen Parlaments stand « und nicht behandelt wurde, berichtet. Es wird also über einen Gesetzesentwurf in einem anderen Land auf einem anderen Kontinent gesprochen, der nicht einmal behandelt worden ist, nur um darauf zu verweisen, dass die Gefahr, dass dieses Gesetz »in der kommenden Legislaturperiode« behandelt werden könnte, besteht: »Es gibt keinen Grund zur Entwarnung. [...] Der Gesetzesentwurf kann unter neuem Namen und veränderten Inhalten sehr wohl wieder eingebracht werden.« Die Unverhältnismäßigkeit, die alleine schon die Relevanz dieser Kurznachricht betrifft, veranschaulicht exemplarisch, wie in und durch eine bestimmte Art und Weise, bestimmte Themen zu setzen oder nicht zu setzen, die Welt in "gut« und »böse« - wie TrIQ es bezeichnen würde - eingeteilt wird.

In der »Out! « wird also nicht, wie in den anderen Projekten, in der Auseinandersetzung mit Rassismus die eigene Gemeinschaft als solidarische Gemeinschaft hergestellt, sondern es werden, im Gegenteil, rassistische Praktiken der Produktion der intoleranten Anderen eingesetzt. Während es sich in diesem Fall recht eindeutig um eine rassistische Praktik handelt, gilt es für die Verhandlungen der anderen Einrichtungen die Frage nach dem Grad der Schließung zu stellen.

\subsubsection{Solidarität heißt, Mehrfachdiskriminierung in den Blick zu nehmen}

Welche Rolle spielen nun Identitäten beziehungsweise Identitätspolitiken für die Konstitution der jeweiligen politischen Gemeinschaften in der Auseinandersetzung mit Rassismus? Auf Ebene der konkreten (Identitäts-)Kategorien, über die und in deren Namen gesprochen wird, nimmt die Villa in Form von expliziten Nennungen Bezug auf die Mehrfachzugehörigkeitsbezeichnung "LGBTIQ*-Geflüchtete«. Diese Bezeichnung wird verwendet, um auf eine bestimmte prekäre Situation einer Gruppe aufmerksam zu machen, die aufgrund einer von der Norm abweichenden sexuellen oder vergeschlechtlichten Lebensweise geflüchtet ist. Ähnlich wie es sich bereits in den Auseinandersetzungen zum Thema Pride gezeigt hat, wird eine Mehrfach-Kategorie verwendet, die nicht einfach nur als Identitäts-Kategorie verstanden wird. Neu an der Herangehensweise, wie die Villa diese Kategorie verwendet, ist der Bezug zum Prekaritätsbegriff. Während die anderen Gruppen eher über die ungleiche Verteilung von Machtverhältnissen sprechen, die über solche Kategorien angeordnet sind, beschreiben im Fall der Villa Mehrfachzugehörigkeiten die ungleiche Verteilung von Prekarität. Damit wird ein Begriff eingesetzt, der erstens nicht an die in der queeren Community äußerst aufgeladenen Privilegien- und »Positionierungs«-Diskurse anknüpft 
und zweitens von Judith Butler explizit als ein Begriff ins Spiel gebracht wurde, der jenseits identitätspolitischer Differenzierungen funktioniert. ${ }^{136}$ Des Weiteren finden sich im Villa-Artikel Selbstbezeichnungen wie »Abartige« und »Mehrheitsangehörige«. Während das »Abartige« zugleich Alliteration und subversive Aneignung einer negativ konnotierten Fremdbezeichnung ist, wird die Bezeichnung »Mehrheitsangehörige« im Zusammenhang mit dem Privilegien-Diskurs verwendet. Mit diesem Begriff wird ein Unterschied zu den geflüchteten LGBTIQs markiert, der sich in Privilegien ausdrückt. Die Bezeichnung »Mehrheitsangehörige« beschreibt, was LGBTIQ-Geflüchtete nicht sind und was sie nicht haben - Privilegien. Die Privilegien werden in dem Diskurs der Villa an die Verpflichtung geknüpft, "sie im Sinne der Betroffenen zu nutzen«. Die Villa schließt sich also ebenfalls den bereits im Kontext der Pride diskutierten Positionierungs- und Privilegien-Diskursen der Critical Whiteness an, indem die Einrichtung sich selbst positioniert und aus dieser Positionierung die Verpflichtung zu handeln ableitet. Während im überwiegenden Teil des Artikels dargelegt wird, dass die (historisch geteilte) Prekarität eine Verantwortung füreinander begründet, wird zum Ende des Diskursfragmentes die Verantwortung zu handeln aus der Position als mehrheitsweiße und damit privilegierte Organisation begründet. Im ersten Fall handelt es sich um einen verbindenden Gemeinschaftsbezug, der ein verantwortungsvolles Handeln begründet. Im zweiteren Fall ist es das Trennende, die Differenz, die zum Handeln verpflichtet. Auch hier stellt sich wieder die Frage, im Namen welcher Betroffenen die Privilegien eingesetzt werden und was die Interessen der Betroffenen sind. Sollen es die Bedürfnisse der Menschen sein, die es, wie TrIQ es im Redebeitrag zum Transgenialen CSD formuliert, am härtesten trifft? Oder sollen die Gruppen, die, wie TrIQ und LesMigraS argumentieren, bisher noch kein Stück vom Rechtskuchen (Entpathologisierung) abbekommen haben, im Zentrum stehen?

Im Villa-Artikel werden die Orte des gemeinsamen Kampfes durch eine Praxis des "Zuhörens « und »Raumgebens « ausgelotet. ${ }^{137}$ Durch eine Praxis des »Zuhörens « ist es der Villa-Argumentation zufolge möglich, »im Sinne der Betroffenen« zu agieren. Im Anschluss an Spivaks Überlegungen ist eine Praxis des Zuhörens eine notwendige Voraussetzung für ein emanzipatorisches Handeln, das jenseits von Paternalismus die Handlungsfähigkeit der Subalternen aktivieren will. Es ist ein Versuch, die abgeschlossenen oder auch ein- und ausschließenden Räume des Sprechens und »Gehörtwerdens« zu öffnen. Um den »Geflüchteten« wiederum zuhören zu können, ist es notwendig, »ihnen [den LGBTIQ-Geflüchteten] Raum zu geben, um ihr Wissen und ihre Erfahrungen einzubringen«. Das »Raumgeben« ist eng verknüpft mit einer Praxis des »Zuhörens«, geht aber auch darüber hinaus. Es ist insofern eine »offene« Praxis, als denen, die keine Räume haben, Räume gegeben werden. Semantisch kann allerdings mit Bezug auf Spivak von einer paternalistischen »Geber*innen«-»Empfänger*innen«-Opposition gesprochen werden. Dabei werden die, denen Räume gegeben werden, auf den Platz der

136 J. Butler: Raster des Krieges, S. 37.

137 Das bedeutet nicht, dass TrIQ und LesMigraS nicht zuhören. Im Cegenteil: als konkrete Interessenvertretung von mehrfachmarginalisierten Gruppen sind sie per se Räume für Mehrfachdiskriminierte und müssen diese nicht erst herstellen. 
Hilfe-Nehmer*innen verwiesen. ${ }^{138}$ Der emanzipatorische Grad der jeweils konkreten Praxis des »Gebens« ist auch daran zu ermessen, inwieweit es sich in eine kapitalistische, rassistische und postkoloniale "Schuldenlogik « einschreibt. ${ }^{139}$

Während die Villa sich also als ein Ort konstituiert, in dem - aus einer Praxis des »Zuhörens« und »Raumgebens « heraus - die »Privilegien im Sinne der Betroffenen« genutzt werden, wird auf der anderen Seite der Kampf um Wohnraum in der Villa-Argumentation erst als der privilegierte Ort des verantwortungsvollen Handelns hergestellt. Es ist zum einen die kohärente Geschichtserzählung und zum anderen die Tatsache, dass das Asylverfahren als Ort möglicher Interventionen verworfen wird, die den Kampf um Wohnraum als privilegierten Einsatzpunkt erst herstellt. Dabei fragt die kohärente Erzählung - an die der Kampf um Wohnraum für Geflüchtete anknüpft - nicht danach, warum und inwiefern sich der Kampf um Wohnraum verändert und verschoben hat. Hieß »Kampf um Wohnraum« in den 1980er-Jahren noch »Häuser besetzen«, so heißt es mittlerweile, Unterstützung vom Staat zu fordern und Spendengelder zu akquirieren, um Mieten zahlen zu können. Die kohärente Erzählung schweigt in Bezug auf ihre Brüche und die zerbrochenen Möglichkeiten ${ }^{140}$ und akzeptiert so auch die aktuellen Möglichkeitsbedingungen politischen Handelns unhinterfragt. ${ }^{141}$ In beiden Fällen ist es allerdings der Staat, der als Produzent von »Ungleichheiten« als Unterstützer*in angerufen und gleichzeitig von der eigenen Verantwortung entlastet wird. Der Kampf um Wohnraum für Geflüchtete ist insofern ein offener, als es sich um ein verantwortliches Handeln im Namen einer gemeinsam geteilten Prekarität handelt. Er ist auch insofern offen, als er Resultat einer Praxis des "Zuhörens« ist. Gleichzeitig wird der konkrete Einsatzpunkt - die Wohnsituation - im Namen einer kohärenten Geschichte ausgelotet.

Auch in Bezug auf die Frage nach der Rolle von Identität in der Aushandlung von Rassismus beim Jugendnetzwerk Lambda BB soll zunächst einmal der Blick auf die Ebene der verwendeten (Nicht-)Identitätsbezeichnungen gelenkt werden, um sich dann generell der Frage nach den Schließungen und Öffnungen der solidarischen Positionierung in der Stellungnahme von Lambda BB zuzuwenden. Die konkret verwendeten Selbstbezeichnungen schließen, ähnlich wie auch bei der Villa, an die Selbstpositionierungen der Critical-Whiteness-Diskurse an. So spricht Lambda BB von der Community und sich selbst als »weiß, christlich, cis-männlich und nicht-behindert dominierte Community«. Wie Lambda BB an bestimmte Aspekte der Critical Whiteness anschließt, wurde bereits im Abschnitt zum CSD ausführlicher diskutiert. In dieser Pressemitteilung werden die

138 Vgl. Gayatri C. Spivak: Righting Wrongs. Unrecht richten, Zürich: Diaphanes 2008.

139 Lorey setzt dieser Schuldenlogik die emanzipatorische Idee von Queeren oder schwarzen Schulden entgegen. Vgl. Isabell Lorey: »Emanzipation und Schulden«, in: Alex Demirovic/Susanne Lettow/Andrea Maihofer et al. (Hg.), Emanzipation. Zu Geschichte und Aktualität eines politischen Begriffs, Münster: Westfälisches Dampfboot 2018, S. 10-15.

140 S. Ahmed: Living a Feminist Life, S. 168.

141 Selbstverständlich würde die prekäre Situation, in einem besetzten Haus zu wohnen, wahrscheinlich nicht oder weniger dazu beitragen, die Geflüchteten im Asylverfahren zu unterstützen. Zudem ist für Menschen ohne Aufenthaltsgenehmigung diese zum Teil »illegale« Form des politischen Protests nicht leistbar. 
Selbstbezeichnungen konkret verknüpft mit einer »Angewiesenheit auf Kritik von unterschiedlichen Bewegungen«, mit einer Verpflichtung zur »Verantwortung«, aber vor allem auch damit, dass Lambda BB nicht »für« LesMigraS sprechen kann, sondern nur "für« sich selbst, »als Teil einer mehrheitlich weißen Community«. Lambda BB kann nicht »für« LesMigraS sprechen (weil Lambda $B B$ »eiß, christlich, cis-männlich und nicht-behindert dominiert « ist) und spricht stattdessen »über« sich selbst. Warum kann Lambda BB aufgrund einer Positionierung nicht »für« LesMigraS sprechen? Diese Verknüpfung geht zurück auf Kritiken an Politiken, die im Namen einer universellen Identität vorgeben, eine Gruppe zu repräsentieren, die sie nicht repräsentieren. Es geht aber auch zurück auf die Kritik eines paternalistischen Sprechens im Namen der anderen, wie es Spivak am Beispiel der Witwenverbrennung ausführt. ${ }^{142}$ Bewusst nicht für eine andere Gruppe zu sprechen, kann in Anlehnung an Spivak auch als Versuch verstanden werden, das »Verstummen der Subalternen« zu beenden. Die Subalternen sind bei Spivak allerdings nicht durch Positionen, wie nicht-weiß, nicht-christlich, nicht-cismännlich usw., bestimmt. Sie stehen zwar in Differenz zu den Eliten, aber in Bezug auf die ihnen zur Verfügung stehenden $»$ Linien der Mobilität «. ${ }^{143}$ Es ist die Tatsache, dass sie nicht sprechen können, die Subalterne zu Subalternen macht, und nicht ihre Position in Bezug auf Geschlecht, Religion, Behinderung und Ethnie. Solche Verknüpfungen von Positionierung und Sprechen werden in antirassistischen Debatten unter anderem dafür kritisiert, dass politische Haltung und subjektive Erfahrung unmittelbar aneinander gekoppelt werden. Dies würde in einer Politik resultieren, in der streng genommen jede*r nur noch für sich selbst sprechen kann:

»Zu behaupten, dass eine politische Haltung unveränderbar an die Position in der Gesellschaft gebunden ist, bedeutet, einen statischen Begriff von Cesellschaft zu vertreten, der die Möglichkeit der Veränderung letztlich ausschließt. Die Gleichsetzung von subjektiver Erfahrung mit politischer Haltung führt dazu, dass am Ende jeder nur für sich selbst sprechen kann - entweder für sich als Individuum oder aber als Vertreterln einer irgendwie gearteten $>$ Hauptkategorie $\ll .{ }^{144}$

Diese Praxis wird als schließend bezeichnet, da sie statisch ist und die Möglichkeit der Veränderung letztlich ausschließt. Zum einen insofern, als gesellschaftliche Veränderungen verschlossen werden, zum anderen insofern, als eine identische Beziehung (Gleichsetzung) von politischer Haltung und subjektiver Erfahrung hergestellt wird. Zugleich kann durch die Haltung, nicht »für« eine andere Gruppe zu sprechen, auch ein paternalistisches Sprechen im Namen dieser Gruppe (hinter dem allzu oft [unbewusst] eigene Interessen stehen) verhindert werden beziehungsweise können die hegemonialen Räume des Sprechens für diejenigen eröffnet werden, die bislang nicht gehört wurden. Was heißt es aber, stattdessen »über« sich selbst oder für die mehrheitsweiße Community zu sprechen? Wird dann nicht doch der Raum eingenommen, um in dem

142 Vgl. hierzu die Ausführungen in Kapitel 1.3.

143 M. d. M. Castro Varela/N. Dhawan: Postkoloniale Theorie, S. 187.

144 J. Karakayali/V. S. Tsianos/S. Karakayali/A. Ibrahim: Decolorise it! Die Rezeption von Critical Whiteness hat eine Richtung eingeschlagen, die die antirassistischen Politiken sabotiert, S. 7. 
Fall sich selbst als solidarisch zu präsentieren? Oder muss eine »selbstkritische« Auseinandersetzung notwendigerweise »öffentlich« ausgetragen werden, um die Selbstkritik - die auch über sich selbst hinausreicht - sichtbar zu machen?

Neben einer Verknüpfung der Position mit den Möglichkeiten des »Sprechens für« spricht Lambda BB auch von einem Wir als "LGBTI* «. Es wird also eine ähnliche Zeichenkette, wie sie LesMigraS oder auch die Villa verwendet, aufgerufen. Im Gegensatz zur Villa und zu LesMigraS bezeichnet das Akronym nicht die Marginalisierungserfahrung, in dessen Namen Forderungen gestellt werden, sondern das eigene Wir. Wir Lambda BB - sind »LGBTI* «. ${ }^{145}$ Lambda BB verwendet die Zeichenkette »LGBTI* ", deren ambivalentes Verhältnis zwischen Schließung und Öffnung bereits diskutiert wurde, um das eigene Wir zu bezeichnen.

Auch im Fall von Lambda BB spielen Verletzungen eine Rolle für die Art und Weise, wie die eigenen solidarischen Politiken begründet werden. Lambda BB solidarisiert sich im Diskursfragment mit einer Gruppe, die Verletzungen aufgrund von Mehrfachzugehörigkeiten ausgesetzt ist. Es ist aber nicht, wie bei der Villa, der Begriff der Prekarität, sondern es sind die Affekte, die das verbindende Moment solidarischer Politiken anrufen. Lambda BB ist solidarisch, weil Lambda BB »beunruhigt « und »erschrocken « ist von dem Rassismus, der sich in den sozialen Netzwerken zeigt. Negative Affekte, ausgelöst durch Verletzungen anderer Körper, stellen eine Grundlage für eine ethische Verantwortung/Verpflichtung füreinander dar, die - wie Butler schreibt - wesentlich durch Medien vermittelt werden kann. Es ist, mit Judith Butler gesprochen, eine durch (soziale) Medien vermittelte Empathie, die aus einer ethischen Verantwortung füreinander Möglichkeiten solidarischer Politiken eröffnen kann. Doch Butler spricht von den medial vermittelten ethischen Verpflichtungen gegenüber denjenigen, die uns »fern« sind. ${ }^{146}$ Was heißt es, wenn sich - wie im Fall von Lambda BB - Hassreden erst in den öffentlichen, für alle sichtbaren Raum der sozialen Netzwerke einschreiben müssen, um solidarische Politiken zu eröffnen? Was bedeutet es, wenn die Verletzung als Zeug*in in den sozialen Medien erst selbst erfahren werden müssen, damit darauf geantwortet wird, obwohl die anderen nahe sind und ständig ${ }^{147}$ von diesen Verletzungen sprechen? Bedeutet das, dass Sprechen von LesMigraS nicht "gehört « wird? ${ }^{148}$ Dass die »hegemonial strukturierten Räume« des »Zuhörens« insofern affirmiert werden, als die, die auf der (hegemonialen) Seite des »Zuhörens« stehen, erst dann zuhören, wenn sie das »Gehörte« medial vermittelt selbst spüren? ${ }^{149}$ Aus der bereits diskutierten Unmöglichkeit,

145 Lambda BB verweist aber auch darauf, dass diese Zeichenkette keine homogene Gruppe bezeichnet: »Als LCBTI* sind wir zwar alle von Diskriminierung betroffen - wir sind aber nicht alle gleich davon betroffen!«

146 J. Butler: Anmerkungen zu einer performativen Theorie der Versammlung, S. 134.

147 Auch in den Jahren vor der Nicht-Teilnahme von LesMigraS gab es Kritik am Lesbisch-schwulen Stadtfest.

148 Durch das »Netzwerk Diskriminierungsfreie Szene für alle« gibt es Möglichkeiten des regelmäßigen Austauschs, und LesMigraS, aber auch »Cladt e.V.«und TrIQ e.V. haben, wie zu Beginn erwähnt, ja auch Pressemitteilungen veröffentlicht, in denen sie über die Verletzungen sprechen.

149 Vielleicht sind die »Rassismen« und »Sexismen«-strukturiert durch die Hatespeech-verstärkenden Möglichkeitsbedingungen sozialer Medien - auf eine andere Art auf die Spitze getrieben worden. So oder so sprechen Gruppen wie »Gladt e.V.« und LesMigraS schon seit Jahren vor al- 
"für « LesMigraS zu sprechen, werden die Affekte der Sorge und Angst nicht auf die Verletzungen und damit eine gemeinsam geteilte Verletzlichkeit bezogen, sondern auf die Community, die als »das, was sie ist«, in Gefahr ist. Ähnlich wie in sogenannten »Politiken mit der Angst« verweisen auch die Ängste und Sorgen von Lambda BB auf ein »Bedrohungsszenario «. ${ }^{150}$ Allerdings ist das, was als »bedroht « inszeniert wird, nicht die »Unversehrtheit « der durch den Hatespeech verletzten Körper - darüber kann Lambda BB als mehrheitsweiße Organisation nicht sprechen -, sondern das, was »bedroht « ist, ist die Community »als das, was sie ist«. Das, was »bedroht« ist, wird selbst als etwas »in sich Abgeschlossenes « beschrieben, als etwas, »das ist, was es ist « und was es in Abgrenzung zu anderen besonders macht. Lambda BB schreibt im Namen einer queeren, offenen Haltung - die Community als Ort der Selbstkritik - eben jene als »das, was sie sind«, fest. Ist dies ein Versuch, die eigenen Forderungen nach Solidarität als »Alleinstellungsmerkmal« in Abgrenzung zu den vielen anderen Communitys, die dieses Merkmal nicht teilen, begehrenswerter zu machen?

Auf der einen Seite ist die Stellungnahme eine solidarische Positionierung mit denjenigen, die rassistische und transphobe Verletzungen erfahren haben. Auf der anderen Seite werden hegemonial strukturierte Anordnungen des Sprechens und Hörens affirmiert, wenn die durch die sozialen Medien vermittelten Verletzungen - und nicht das, was LesMigraS und andere sagen - in den Vordergrund gestellt werden. Durch eine affektiv angerufene Sorge um die Community, die als »das, was sie ist «, in Gefahr ist, wird eine solidarische Community gefordert. Die Community wird in neoliberaler Manier "Exklusivität als Marke« - verkauft, um den Forderungen nach Selbstkritik Gewicht zu verleihen. Gleichzeitig ist das, was Lambda BB der Community verkaufen will - was sie von ihr (ein-)fordert, nämlich eine Öffnung der Community -, Solidarität im Sinne einer Gemeinschaft, die ein offener Ort »kollektiver Auseinandersetzungen« ist.

Auch TrIQ fordert eine »offenere«, solidarische Community und stellt sich (ähnlich wie Lambda BB) in Abgrenzung zu denen, die durch diese Forderungen angerufen werden, als bereits solidarische Gemeinschaft her. Im Gegensatz zur Villa und zu Lambda BB wird nicht das LGBTIQ*-Akronym verwendet. TrIQ spricht lediglich von Sexarbeiter*innen sowie geflüchteten oder arbeitslosen Personen als Gruppen, die einen Platz in der Community haben sollten. Die Haltung, die TrIQ gegenüber den Medien vertritt und von der Community fordert, schließt an postkoloniale Kritiken der Produktion des »intoleranten Anderen«, die zumeist entlang der Nord-Süd-Achse verläuft, an. Allerdings macht auch diese, an Saids Denken anschließende Dichotomie die Komplexität der Verhältnisse unsichtbar. Eine solche, auf postkolonialen Interventionen beruhende Kritik ist wichtig und notwendig, verweist sie doch darauf, wie queere Politiken und Errungenschaften auf Kosten derjenigen gehen, die in diesen Narrativen als »schlecht« gel-

lem rassistische und transphobe Übergriffe am Lesbisch-schwulen Stadtfest an. Lambda BB hingegen betont in der solidarischen Pressemitteilung gleich zweimal, dass es »insbesondere « über die »Online-Kommentare« erschrocken und schockiert sei, anstatt die Ängste und Sorgen direkt auf die Erzählungen ebendieser Gruppen zu beziehen.

150 H. Mitterhofer-M. Fritsche-T. Vogler-F. Madlung-M. Brandmayr: >UND WER SCHÜTZT UNS BÜRGER????<, S. 96. 
ten. TrIQ kritisiert hier also eine auf Schließung abzielende Praxis. Allerdings laufen solche Kritiken Dhawan zufolge auch Gefahr, umgekehrte Einseitigkeiten zu produzieren, sofern sie es unmöglich machen, Homophobie und Transphobie im globalen Süden $\mathrm{zu}$ benennen. Inwieweit die Forderungen nach solidarischen Politiken einer solchen Einseitigkeit entkommen, ist anhand der Diskursfragmente schwer zu beurteilen. ${ }^{151}$ Allerdings liegt der Fokus der Intervention von TrIQ auf der Community im globalen Norden. Es wird also nicht über den globalen Süden gesprochen. Die Community im globalen Norden wird dafür kritisiert, dass sie der Produktion des »intoleranten Anderen« auf den »Leim« geht. Statt den Medien auf den »Leim zu gehen« wird von ebendieser gefordert, Räume zu schaffen, in denen mehr Menschen sichtbar und hörbar sind. Ähnlich wie Lambda BB, fordert allerdings auch TrIQ die Community als das, was TrIQ ist, beziehungsweise stellt die Einrichtung sich in Abgrenzung zur Community als solidarisch her.

An diese Argumentation schließt auch LesMigraS - wie bereits im Diskursfragment zur Pride dargelegt - an. Expliziter als im Diskursfragment zur Pride wird in dieser Auseinandersetzung deutlich, warum die Community ein zentraler Ort des Politischen bei LesMigraS ist. LesMigraS ruft die Community als mögliches "Zuhause« an. Ausgehend von den Erfahrungen der von Gewalt und Diskriminierung Betroffenen, mit denen LesMigraS seit Jahren in der eigenen Arbeit konfrontiert ist, hat die Einrichtung ein Gewaltverständnis entwickelt, demzufolge die Community als eine Art »Prisma« fungiert, das manche Gewalt- und Diskriminierungsverhältnisse abschwächt, andere jedoch durchlässt oder sogar verstärkt. ${ }^{152}$ Gleichzeitig ist ein wichtiger Bestandteil der Arbeit von LesMigraS - auch das geht auf die Erfahrungen, die in der Arbeit gemacht wurden, zurück -, potentielle Unterstützer*innen von Gewalt- und Diskriminierungsbetroffenen anzurufen. ${ }^{153}$ LesMigraS fordert also eine Community, die solidarisch ist, weil die Erfahrungen gezeigt haben, dass die Community ein wichtiger Zufluchts- und Unterstützungsort für Menschen, die Gewalt und (Mehrfach-)Diskriminierung erfahren, sein kann. Die - wie bereits in der Analyse zur Pride gezeigt wurde - zum Teil auf Schließung abzielenden Forderungen an die Community sind Resultat eines aus der Arbeit mit den Betroffenen entstandenen Wissens, das wiederum das Gewaltverständnis von LesMigraS prägt. ${ }^{154}$

Im Gegensatz $\mathrm{zu}$ Lambda BB und TrIQ wird in der Pressemitteilung zum "IDAHoT_I « die Community nicht nur aufgefordert, in Bezug auf sich selbst solidarisch zu sein, sondern auch solidarisch die Kämpfe der anderen zu unterstützen. Solidarität geht dem Verständnis von LesMigraS zufolge über eine Praxis der Selbstkritik hinaus und bedeutet, kollektiv gegen strukturelle Beschränkungen zu handeln. Auch hier werden der Staat, die Medizin und das Recht als »zentraler Hort des Per- 
sonseins« angerufen, der, Dhawan folgend, als ein Pharmakon - zugleich Gift und Medizin - bezeichnet werden kann. ${ }^{155}$

Während die Berliner Projekte Solidarität von der Community fordern und sich in und durch diese Forderung in doppelter Hinsicht selbst als solidarisch konstituieren, konstituiert die Milchjugend sich insofern als solidarisch, als sie selbstkritisch Rassismus reflektieren will. Indem die Milchjugend an der »Oberfläche«, Bezug nehmend auf eigene Erfahrungen, über Rassismus spricht, wird zunächst all jenen eine Identifikationsmöglichkeit eröffnet, die sich noch nicht mit diesem Thema auseinandergesetzt haben. Die Milchjugend konstituiert sich so als ein Wir, das noch zu lernen hat und gleichzeitig diese Räume des Lernens herstellt. Die durch die Milchjugend eröffneten Möglichkeiten der Reflexion bleiben - wie im Editorial der entsprechenden »Milchbüechli«-Ausgabe angekündigt - an der »Oberfläche«, was auf der einen Seite den Zugang für viele Menschen eröffnet, auf der anderen Seite aber auch bestimmte Möglichkeiten des Denkens verschließt. Indem eine Analogie zwischen den selbst erfahrenen Stereotypen und denen, die Menschen aufgrund von Rassismus machen, gezogen wird, ist die Nachvollziehbarkeit bestimmter Mechanismen von Rassismus niederschwellig angelegt. Verschlossen bleiben in dieser nachvollziehbaren Analogie allerdings die wesentlichen Unterschiede zwischen der Diskriminierung aufgrund von »Falschsexualität « und Rassismus (beispielsweise die Frage der Sichtbarkeit, die Geschichte, Homonormativität, Homonationalismus) und die vielfältigen Mechanismen jenseits von »Klischees«, durch die Normen und Unterdrückungsverhältnisse wirken.

Solidarität wird in diesen queeren Diskursen eng verknüpft mit der Thematisierung von Mehrfachdiskriminierung, im Besonderen mit der Thematisierung von Rassismus, und so mit (neuen) Bedeutungen versehen. Gemeinsam ist allen fünf Projekten, dass Solidarität in Bezug auf Rassismus bedeutet, einen kritischen Blick auf die rassistischen Praktiken der eigenen Gemeinschaft zu werfen. ${ }^{156}$ Solidarität bedeutet also, mit Judith Butlers Worten, offen zu sein für Selbstkritik. Solidarität, wie es von den Projekten verstanden wird, verpflichtet aber auch, über die kritische Auseinandersetzung in der eigenen Gruppe hinaus zu handeln. Solidarität bedeutet, gemeinsam Forderungen zu stellen und dabei auch, wie im Fall der Berliner Projekte, eine solidarische Community zu fordern. Durch diese Forderungen nach einer solidarischen Community konstituieren sie sich selbst in doppelter Hinsicht als solidarisch: zum einen, indem sie sich in Abgrenzung zur Community als bereits solidarisch herstellen, und zum anderen, indem sie sich dadurch als solidarisch konstituieren, dass sie eine solidarische Community einfordern. Solidarität bedeutet in den Auseinandersetzungen der queeren Projekte aber auch selbst solidarisch zu handeln. Das geschieht auf unterschiedlichen Ebenen: Es sind Kämpfe um Wohnraum und Forderungen an das Recht, den Staat oder die Medizin. Unterschiedlich ist auch der gemeinsame Nenner, unter dem diese Forderungen

155 N. Dhawan: Homonationalismus und Staatsphobie: Queering Dekolonisierungspolitiken, QueerPolitiken dekolonisieren, S. 49.

156 Eine Ausnahme stellt die Verbandszeitschrift des Jugendnetzwerks Lambda dar, in der anstatt rassistische Ausschlüsse zu thematisieren, diese selbst produziert werden. 
gestellt werden: Es ist Prekarität, die bedrohte Exklusivität der Community, die besondere strukturelle Diskriminierung von Mehrfachmarginalisierten, gemeinsam geteilte Diskriminierungserfahrungen oder das gemeinsame Wissen darum, dass Transphobie, Homophobie und Rassismus auch im Westen töten. Die queeren Projekte versuchen sich in der Auseinandersetzung mit Rassismus also insofern als offene Orte herzustellen, als sie sich als solidarische Gemeinschaften konstituieren. Dabei besteht eine gemeinsam geteilte Idee von Solidarität als selbstkritische Auseinandersetzung mit Rassismen in der eigenen Gemeinschaft, die zum Teil auf unterschiedliche Art an intersektionale, postkoloniale und Critical-Whiteness-Diskurse anschließen. Manche Projekte beziehen sich auf Privilegien- und Selbstpositionierungs-Diskurse, andere sprechen davon, »zuzuhören« und »Räume zu geben«. Auch im gemeinsamen solidarischen Handeln über die eigene Gemeinschaft hinaus werden unterschiedliche Fokusse gesetzt, die zum Teil auch mit den konkreten, projektspezifischen Konstellationen zusammenhängen. Die Berliner Projekte sind sehr auf das Ziel einer solidarischen Community fokussiert, was sicherlich auch an der Berlin-spezifischen Community-Situation liegt. Die Villa bezieht sich auf die eigene Vergangenheit als Hausbesetzungsprojekt und die Milchjugend knüpft an eine Gemeinschaft an, in der Auseinandersetzungen mit Rassismus aus verschiedenen Gründen noch nicht derart präsent sind wie in den anderen Einrichtungen.

\subsection{Das Coming-out: Befreiung oder Geständnispraxis?}

Die fünf im Zentrum der Analyse stehenden Projekte sind nicht nur Projekte, die öffentlich am politischen Leben teilhaben, indem sie, wie beispielsweise auf der Pride, auf der Straße erscheinen, sondern auch Orte der kollektiven Unterstützung und des Austauschs. Die Austauschorte sind zum einen Räume, in denen ein gemeinsames politisches Selbstverständnis verhandelt wird oder eben ein selbstkritischer Umgang mit Rassismus möglich ist. Zum anderen sind es aber auch Orte, die an dem individuellen Wohlbefinden derjenigen Menschen, die Teil der Projekte sind, ausgerichtet sind. Beratungen, Workshops, Gruppen und eigene Zeitschriften stellen konkrete Formen solcher kollektiven Austausch- und Unterstützungsorte dar. Mit Ausnahme der Milchjugend gibt es in allen Projekten Beratungsangebote. Die Projekte stellen auch die Infrastrukturen für Gruppentreffen bereit, die zumeist um bestimmte Themen angeordnet sind. Es gibt informelle Austauschorte, wie beispielsweise Cafés oder regelmäßige Treffen in einer Bar, und in vielen Projekten werden (Empowerment-)Workshops angeboten. Die beiden queeren Jugendzeitschriften »Out! « und »Milchbüechli« informieren und diskutieren nicht nur aktuelle Ereignisse, sondern sind auch insofern Austausch- und Unterstützungsorte, als die Jugendlichen diese Medien nutzen, um ihre Erfahrungen $\mathrm{zu}$ teilen.

Orte des Austauschs, die auf das individuelle Wohlbefinden ausgerichtet sind, waren schon immer Bestandteil geschlechterpolitischer sozialer Bewegungen. So spielten beispielsweise in der zweiten Frauenbewegung die sogenannten Selbsterfahrungsgruppen eine wichtige Rolle. In diesen Gruppen ging es zunächst darum, gemeinsam, durch den Austausch von Erfahrungen, ein Bewusstsein für strukturelle Unterdrückung zu 
erarbeiten. Diesen Gruppen wurde eine therapeutische Wirkung, vor allem aber - insbesondere durch ihr Solidarisierungspotential - eine politisierende Wirkung zugesprochen. ${ }^{157}$ Seit den Selbsterfahrungsgruppen der Frauenbewegung hat es, bis hin zu den hier betrachteten queeren Projekten, einige Verschiebungen gegeben. ${ }^{158}$ So wurden die Beratungs-, aber auch die Austauschräume zum Teil professionalisiert und institutionalisiert. ${ }^{159}$ Die gemeinsamen Erfahrungen sind auch in den queeren Projekten noch Ausgangspunkt der Beratungs- und Austauschgruppen, aber sie werden zumeist eher mit dem Ziel verhandelt, die individuelle Handlungsmächtigkeit der Betroffenen zu stärken. ${ }^{160}$ In der Art und Weise, wie diese geteilten Erfahrungen zum Ausgangspunkt dieser Räume gemacht werden, haben die Austausch- und Unterstützungsräume auch eine gemeinschaftsbildende Funktion. Sie dienen sowohl als »Konstituierung und interne Selbstdefinition der Gruppe wie als Symbol ihrer Außendarstellung ${ }^{16}{ }^{16}$ Gleichzeitig eröffnen sie, in der Art und Weise, wie diese Räume angeordnet sind, auf einer individuellen Ebene bestimmte Möglichkeiten, zum Subjekt zu werden, andere bleiben undenkbar. Das bedeutet, das Wir der Projekte konstituiert sich nicht nur über die Auslotung der Frage, wie diese im Feld des Politischen in Erscheinung treten und wie dieses Wir selbst offener gestaltet werden kann, sondern auch über die Art, wie Austausch- und Unterstützungsorte im Namen eines individuellen Wohlbefindens angeordnet sind. Sie konstituieren sich insofern als ein spezifisches Wir, als an diesen Austauschorten bestimmte Möglichkeiten des Seins als denkbar hergestellt werden und andere undenkbar bleiben. In der Mehrheit der Projekte ist dabei das Coming-out immer noch ein zentrales Thema, um das sich die jeweiligen Austausch- und Unterstützungsorte anordnen. Insbesondere bei LesMigraS, das in einer Pressemitteilung erklärt, warum es den Coming-out-Begriff ablehnt, scheint allerdings eher der Empowerment-Begriff die Anordnung der individuellen Austausch- und Unterstützungsorte zu bestimmen. ${ }^{162}$

Das Coming-out und dessen zentrale Rolle in sexualpolitischen Bewegungen lassen sich vor allem auf die »Gay Liberation« der 1970er-Jahre zurückführen. Die Bezeichnung Coming-out geht auf die englischsprachige Redewendung »Coming-out of the

157 Angelika Wagner: »Bewußtseinsveränderung durch Emanzipations-Cesprächsgruppen«, in: HansDieter Schmidt-Christiane Schmerl-Astrid Krameyer et al. (Hg.), Frauenfeindlichkeit. Sozialpsychologische Aspekte der Misogynie, München: Juventa-Verlag 1973, S. 143-160.

158 Grundsätzlich haben sich viele queere Räume zu sogenannten Safe Spaces oder Care-Räumen entwickelt.

159 Mit Ausnahme der Milchjugend, die mittlerweile über ein kleines Büro verfügt, haben alle Projekte eigene Räume und bieten, mit Ausnahme der Villa, »bezahlte« Beratung an.

160 Empowerment ist ein Begriff, der solche Orte des Austauschs mittlerweile prägt. In der Analyse zur Pride wurde bereits ausgeführt, dass mit dem aus der Schwarzen Bürgerrechtsbewegung kommenden Begriff die Stärkung der individuellen Handlungsmächtigkeit der von struktureller Diskriminierung Betroffenen gemeint ist.

161 Carolin Küppers-Martin Schneider (Hg.): Orte der Begegnung - Orte des Widerstands. Zur Geschichte homosexueller, trans*geschlechtlicher und queerer Räume, Hamburg: Männerschwarm Verlag 2018, S. 9.

162 Auch bei TrlQ und Lambda BB ist der Empowerment-Begriff wichtig. Allerdings ist bei Lambda BB das Coming-out immer noch zentral. Im Fall von TrlQ spielt beides eine Rolle. Der EmpowermentBegriff bei LesMigraS wurde bereits in der Analyse zur Pride ausführlicher betrachtet. 
closet« zurück. Der Begriff wurde von der Tradition des »coming-out ball« übernommen, »der US-amerikanischen Variante des Debütantinnenballs, bei der junge Frauen aus der Oberschicht zum Zweck der >Brautschau in die feine Gesellschaft eingeführt werden ${ }^{163}$ "The closet « stand metaphorisch für das »[V]erstecken der Homosexualität« in der Privatsphäre und den Subkulturen. Mit der »Gay Liberation« wurde das Coming-out als öffentliche, politische Strategie zu einem zentralen Moment der homosexuellen Befreiungsbewegung, von der sich Anerkennung durch Sichtbarkeit erhofft wurde: »Out of the closet into the streets!« oder »Out and Proud« waren zentrale Slogans dieser bewegten Zeit. ${ }^{164}$ Auch im queeren US-amerikanischen Aktivismus der 8oer-Jahre spielte Coming-out als politische Strategie eine Rolle. So performten Gruppen wie »Queer Nation« Coming-outs auf dramatisierende Weise in Form von »Kissins « oder "Die-ins « an öffentlichen Orten. ${ }^{165}$ Auch Outing im Sinne eines Fremdoutings war eine - wenn auch kontrovers diskutierte - Strategie in der Aids-Krise. ${ }^{166}$ Das Coming-out hatte oder hat in queeren Kontexten vor allem zu Beginn immer (auch) eine politische Dimension, die historisch auch notwendig war, um beispielsweise öffentlich eine Betrauerbarkeit oder »unbetrauerbare Verluste« (»Die-in«) einzufordern. ${ }^{167}$ In queeren Diskursen wird aber auch Kritik geübt an den »Zwängen, Ausschlüssen und Normalisierungen«, die mit dem Coming-out einhergehen. ${ }^{168}$ So »bejaht « das Comingout zum Teil nicht nur eindeutige Identitäts-Kategorien, sondern schließt oft auch an Diskurse der sexuellen Befreiung an, denen eine einseitige Vorstellung von einer Unterdrückung der Sexualität vorausgeht. Michel Foucault hat mit dem Erscheinen des Bandes »Sexualität und Wahrheit« die Illusion genommen, dass man »zur Macht nein sagt, wenn man zum Sex ja sagt«. Man folgt vielmehr dem »Lauf des allgemeinen Sexualitätsdispositivs «. ${ }^{169}$ Noch komplexer wird es, wenn Coming-out-Politiken aus postkolonialer Perspektive betrachtet werden. »Das Subjekt des Coming-out « ist auch - so Nikita Dhawan - durch eine »koloniale« Geschichte gekennzeichnet. ${ }^{170}$ Wie bereits in der Analyse zur Mehrfachdiskriminierung angeklungen ist, werden koloniale Verhältnisse reproduziert, indem queere Menschen aus dem globalen Norden in Abgrenzung zu denen aus dem globalen Süden als stolze, geoutete Subjekte hergestellt werden.

Neben dieser politischen Ebene, die in den aktivistischen Kontexten immer auch eine Rolle spielt, ist das Coming-out aber auch auf einer »individuell psychosozialen

163 V. Woltersdorff: Coming out, S. 45.

164 Volker Woltersdorff: „Coing Public - Coing Media. Über den medialen Wandel schwuler Comingout-Inszenierungen seit Stonewall«, in: Susanne Regener-Katrin Köppert (Hg.), Privat-öffentlich. Mediale Selbstentwürfe von Homosexualität, Wien: Turia + Kant Verlag 2013, S. 89-110, hier S. 90.

165 Ebd., S. 100.

166 So wurden beispielsweise Politiker*innen geoutet, die »antischwule« Politiken betrieben. Auch in der deutschen schwulen Bewegung hat es »Outings« von homosexuellen Prominenten gegeben. Andreas Heilmann: Normalität auf Bewährung. Outings in der Politik und die Konstruktion homosexueller Männlichkeit, Bielefeld: transcript Verlag 2011, S. 134.

167 J. Butler: Psyche der Macht, S. 139.

168 V. Woltersdorff: Going Public - Coing Media, S. 100.

169 M. Foucault: Der Wille zum Wissen, S. 151.

170 María d. M. Castro Varela-Nikita Dhawan: »Spiel mit dem >Feuer - Post-Kolonialismus und Heteronormativität«, in: Femina Politica - Zeitschrift für feministische Politikwissenschaft 14 (2005), S. 47-58, hier S. $55 f$. 
Ebene« Thema: Unter Coming-out versteht Andreas Heilmann den »individuellen psychosozialen Prozess der homosexuellen Identifikation [...], mit der Offenbarung der sexuellen Identität im engen sozialen Umfeld «. ${ }^{171}$ In psychosozialen Diskursen wird zumeist zwischen dem Coming-out to (dem äußeren Coming-out) und dem Comingout (dem inneren Coming-out ${ }^{172}$ unterschieden. Während bei ersterem das Comingout einen anderen (eine Gruppe oder Personen) als Adressat*innen hat, bezieht sich letzteres auf den Zeitpunkt, an dem mensch seine »eigene Identität « entdeckt. ${ }^{173}$ Die Notwendigkeit des Coming-out to ist daran geknüpft, dass die körperliche Erscheinung nicht unmittelbar die eigene Identität oder sexuelle Orientierung widerspiegelt. In diesem Fall werden zumeist als »normal« geltende vergeschlechtlichte oder sexuelle Identitäten ${ }^{174}$ angenommen: »labels of non-disabled and heterosexuality are always presumed unless otherwise stated «. ${ }^{175}$ Ein individuelles Coming-out ist also nur Thema für solche Körper, die als »normal« gelesen werden und die anders sichtbar sein wollen. Ein Spezifikum im Kontext von queerness und disability ist, dass die jeweilige Zugehörigkeit zumeist nicht mit der "Geburtsfamilie« geteilt wird. ${ }^{176}$ Entsprechend braucht es andere Orte, an denen die Erfahrungen geteilt und Unterstützung gesucht werden kann. Oft ist die Community ein solcher Austausch- und Unterstützungsort.

Bei allen fünf Projekten spielt das Coming-out weniger als politische Strategie eine Rolle, sondern eher als sogenanntes individuelles Coming-out to. Die politischen Strategien der jeweiligen Projekte setzen zwar häufig ein Coming-out voraus beziehungsweise sind damit verknüpft - Politiken der Sichtbarkeit sind oft zentraler Bestandteil der Projekte (z.B. »falschsexuelle« Stadtspaziergänge, »Kiss-ins«, Pride-Paraden, das Lesbisch-schwule Stadtfest u.v.m.) -, das Sprechen darüber wird aber selten explizit mit der Frage nach dem Coming-out in Verbindung gebracht. In den beiden Jugendzeitschriften »Out!« und »Milchbüechli« ist das Coming-out ein sehr zentrales Thema. Aber auch in den Räumen der Villa und von Lambda BB treffen sich regelmäßig Gruppen, die sich explizit zum Coming-out austauschen, und auch die Beratung dieser beiden Projekte hat einen Fokus auf das Coming-out. Während TrIQ auf der Website vom Coming-out als wichtigem psychosozialem Thema spricht, sind die Austauschgruppen um andere Themen ${ }^{177}$ angeordnet. ${ }^{178}$ LesMigraS ordnet die eigenen Unterstützungs- und

171 A. Heilmann: Normalität auf Bewährung, S. 136.

172 Ellen Samuels: »My Body, My Closet. Invisible Disability and the Limits of Coming-Out Discourse«, in: GLQ: A Journal of Lesbian and Gay Studies 9 (2003), S. 233-255, hier S. 237.

173 Ebd., S. 233.

174 Aber auch Körper, wie Samuels in Bezug auf disability zeigt.

175 E. Samuels: My Body, My Closet. Invisible Disability and the Limits of Coming-Out Discourse, S. 235.

176 Ebd., S. 234.

177 Beispielsweise »Passing«, Trans* im Alter oder Weder" Noch*.

178 Inwieweit das Coming-out in der psychosozialen Beratung eine Rolle spielt, ist auf der Website nicht ersichtlich. Cleichzeitig ist das von TrIQ produzierte Text- und Bildmaterial weniger auf Themen die eigenen Austauschräume betreffend angeordnet, sondern mehr um die »Sensibilisierung« von Trans*-Themen nach »außen« (z.B. Mediziner*innen, Medien, Therapeut*innen) sowie die Einforderung von konkreten Rechten (Entpathologisierung, Menschenrechte). Das hängt vielleicht auch damit zusammen, dass es in der Gesellschaft insgesamt noch sehr wenig Wissen und Sensibilität für Trans* und Inter* gibt und dass diese, wie TrlQ selbst auch schreibt, medizinisch immer noch bevormundet und pathologisiert werden. 
Austauschorte eher um den Empowerment-Begriff an, positioniert sich in einer Pressemitteilung aber auch zum Coming-out anlässlich des Internationalen Coming Out Day. ${ }^{179}$ Bei den meisten Projekten ist das Coming-out ein Thema, um das sich diese Austausch- und Unterstützungsräume anordnen.

Im Folgenden soll nun ausführlicher dargestellt werden, welche Rolle das Comingout bei den jeweiligen Gruppen und deren Konstitution als Gemeinschaft spielt. Besonders zentral ist das Coming-out in den beiden Jugendprojekten und insbesondere in den von den Jugendlichen produzierten Zeitschriften. Das liegt zum einen an der Zielgruppe, für die das Coming-out aufgrund des Alters ein zentrales Thema ist. Comingouts finden mittlerweile zunehmend im jüngeren Alter statt. ${ }^{180}$ Hinzu kommt, dass Jugendliche nicht in selbstgewählten Beziehungs- und Wohnverhältnissen leben, ${ }^{181}$ was, wie in den folgenden Ausführungen deutlich wird, das Coming-out in bestimmten Situationen notwendiger macht. Zum anderen liegt es aber auch am Medium - einer projekteigenen Zeitschrift -, das die Möglichkeit bietet, Erfahrungen auszutauschen, während die anderen Medien eher Positionierungen (z.B. Stellungnahmen, Redebeiträge, Website), Aufklärungen (Broschüren, Flyer, Plakate, Website) oder Veranstaltungsbewerbungen (Flyer, Plakate, Website) möglich machen. In den beiden Zeitschriften »Milchbüechli« und »Out!« spielt das Coming-out eine zentrale Rolle. Aufgrund dieser Repräsentativität wurde je ein Artikel aus jeder Zeitschrift für die feinanalytische Darstellung ausgewählt.

\subsubsection{Die Anerkennung der Schuld}

Für das Jungendnetzwerk Lambda BB ist das Coming-out, neben dem Kampf um Räume und finanzielle Ressourcen, ein zentraler Aspekt. Vor allem im Rahmen der Beratung unterstützt das Jugendnetzwerk Lambda BB Jugendliche zum Thema Coming-out. Lambda bietet unter der Bezeichnung »In und Out«-Beratung sowohl "professionelle« als auch peer-to-peer-Beratung an. Auch auf der Website ist das Thema Coming-out präsent. ${ }^{182}$ Dort gibt es Hinweise und Informationen für »Mädchen, Jungen und Trans* « rund um das Coming-out. Das Coming-out wird auf der Homepage zunächst in Abgrenzung zum Verständnis von »out « sein als nicht »in« sein - also als »uncool« sein - erklärt. Es wird aber auch als ein Akt des »Bekennens« definiert: »Es bedeutet, dass diese sich $\mathrm{zu}$ ihrer Sexualität bekennen, vor sich selbst (inneres $\mathrm{CO}$ ) und vor anderen (äußeres CO). ${ }^{183}$ Ähnlich zentral wie für die (Beratungs-)Arbeit des Jugendnetzwerks Lambda BB

179 Den Internationalen Coming Out Day, an dem Menschen zum Coming-out ermutigt werden sollen, gibt es seit 1987 in den USA und wird mittlerweile auch in Europa gefeiert. V. Woltersdorff: Going Public - Going Media, S. 97 V. Woltersdorff: Going Public - Coing Media, S. 97.

180 Vgl. ebd.

181 Die Villa thematisiert beispielsweise den Arbeitsplatz, an dem ebenfalls die Beziehungen oft nicht selbstgewählt sind, als Ort des (un-)möglichen Coming-out.

182 Die Website des Jugendnetzwerks Lambda BB informiert vor allem über die eigene Einrichtung und deren Angebote. Die Coming-out-Rubrik ist die einzige Seite auf der Website, die bereits direkt Informations- und Aufklärungsmaterial zu einem bestimmten Thema bereitstellt.

183 Jugendnetzwerk Lambda:BB: Coming-out, https://www.lambda-bb.de-beratung-coming-out vom 24.11.2020. 
ist das Coming-out auch in der Zeitschrift des Bundesverbandes - „Out!«. Bereits der Name der Zeitschrift nimmt Bezug auf das Coming-out. Entsprechend ist die gesamte Zeitschrift grundlegend um das Coming-out angeordnet. Besonders in den Jahren 2010 und 2011 wird fast immer und überall über das Coming-out geschrieben, aber auch in den folgenden Jahren ist das Coming-out sehr präsent: Nicht nur in der Coming-out-Rubrik, in fast jedem Artikel wird das Coming-out thematisiert. Ist das Thema der Zeitschrift beispielsweise »Sport«, wird über geoutete Sportler"innen geschrieben, ist es das Thema »Stadt/Land «, wird das Coming-out auf dem Land behandelt, ist es das Thema »Familie«, wird über das Coming-out in der Familie geschrieben, zum Thema »Lernen« berichten Lehrer*innen von ihrem Coming-out und wenn das Thema der Zeitschrift »Geheimnis« ist, dann geht es um das Geheimnis, das mensch vor dem Comingout hütet. Auch in der News-Rubrik »Denkwürdiges« wird immer wieder davon erzählt, welche bekannten Personen sich geoutet haben, es werden Filme und Bücher rezensiert, in denen das Coming-out zentral ist, und auch in den Kolumnen kommt dieses Thema vor.

Der für die Feinanalyse ausgewählte Artikel ist in der »Out!«-Ausgabe 14 im Herbst 2010 erschienen. ${ }^{184}$ Der Themenschwerpunkt dieser Ausgabe ist »Wohnen«. In allen Artikeln zum Thema »Wohnen« wird auch das Coming-out zum Thema gemacht. So wird beispielsweise die Frage, ob mensch sich bei der WG-Besichtigung »outen« sollte, diskutiert und über eine "Lesben-WG« berichtet, bei der, auf der Suche nach einer neuen Mitbewohner*in, die Frage nach dem Outing aufgekommen ist. In der Erzählung über das erste Zusammenziehen mit dem Partner wird das (Zwangs-)Outing bei der gemeinsamen Wohnungssuche thematisiert und auch im Artikel über eine Person, die wieder zurück zu den Eltern auf den Bauernhof zieht, wird zu Beginn von der anfänglichen Angst, sich vor den Eltern zu outen, geschrieben. Der für die Feinanalyse ausgewählte Artikel trägt das Outing sogar im Titel. Es geht um die Frage, wie es ist, nach dem Coming-out zu Hause zu wohnen. Der Artikel »GEOUTET ZUHAUSE WOHNEN « ${ }^{185}$ ist auf einer A4-Seite abgedruckt. Oben rechts befindet sich ein Bild von einem roten Sessel und einer Pflanze in einer Wohnung, das auf ein gemütliches Zuhause verweist. Der Artikel ist in insgesamt zwölf Abschnitte unterteilt. Die Autorin ist 18 Jahre alt und keine der prägenden Figuren der »Out!«. Das ist für diese Zeitschrift charakteristisch: In der »Out! « schreiben immer wieder Personen vereinzelt Artikel, in denen sie ihre eigenen Erfahrungen und Erlebnisse teilen. In dem nun analysierten Artikel wird von den negativen wie positiven Erfahrungen nach dem Coming-out in der Familie berichtet.

Im ersten Abschnitt beschreibt die Autorin zunächst ihre Überraschung darüber, dass nach dem Coming-out die »Probleme« erst angefangen haben. Das Coming-out als ein Akt, in dem »man sich seinen liebsten Menschen offenbart«, könne dazu führen, dass diese plötzlich alles in einem »anderen Licht sehen« und »falsch« oder anders »interpretieren«.

$\mathrm{Zu}$ Beginn des Abschnitts werden »die Probleme«, um die sich der Artikel dreht, kontextualisiert, indem eine Unterscheidung zwischen dem konkreten Akt des Com-

184 Jugendnetzwerk Lambda: Wohnen, Out! 2010.

185 Jugendnetzwerk Lambda: »Ceoutet Zuhause wohnen«, in: Wohnen, Out! 2010. 
ing-out - in dem Fall bei der Familie - und dem »Post-Outing« - die Phase, in der die geoutete Person weiterhin mit der Familie zu Hause wohnt - gemacht wird. Durch diese Unterscheidung wird das Coming-out nicht als Prozess, der auch das von der Autor"in als »Post-Outing « bezeichnete Leben beinhalten würde, sondern als einmaliger und abgeschlossener Akt festgeschrieben. Die »Probleme« ereignen sich demzufolge in einer ganz bestimmten, für Jugendliche spezifischen Situation: im Coming-out gegenüber der Familie in einer Zeit, in der mensch noch zu Hause wohnt. Diese Konstellation beinhaltet mehrere Spezifika, die im Text unbenannt bleiben. Zum einen die Tatsache, dass die Familie oft nicht die Erfahrung, in Bezug auf Geschlecht oder Sexualität von der Norm abzuweichen, teilt. Zum anderen befinden sich die oft noch nicht volljährigen Jugendlichen in einem Abhängigkeitsverhältnis gegenüber den Eltern und haben zumeist noch nicht die Möglichkeit, sich ihre Wohnsituation selbst auszusuchen. Der einleitende Abschnitt markiert das Thema des Artikels, das »Post-Outing«-Leben, mit dem die Probleme erst anfangen: »War das Outing dann nämlich endlich durch, fingen die >Probleme für mich erst an. "Auf die Erfahrungen der Autor*in Bezug nehmend, wird die Themensetzung des Artikels begründet: »Ich persönlich machte mir gerade über diesen Punkt nicht wirklich Gedanken, war ich doch mehr mit dem >Wie? Wann? Wo?« meines Outings beschäftigt und wurde in meinem >Post-Outing<-Leben in vielerlei Hinsicht überrascht.« Implizit wird hier das Ziel oder der Zweck des Artikels festgeschrieben: Es geht darum, andere Jugendliche auf die Überraschung vorzubereiten, auf die die Autor*in nicht vorbereitet war.

Nachdem das Thema und der Zweck des Artikels dargelegt wurden, wird auf den Argumentationsverlauf des Textes verwiesen: "Sich zu outen, während man noch $\mathrm{zu}$ Hause wohnt, hat Vor- und Nachteile. Anders als jetzt zu erwarten, beginne ich ausnahmsweise mal mit den Nachteilen. «Hier wird ein Bruch mit Coming-out-Berichten, die sonst mit den Vorteilen beginnen würden, hergestellt. Mit den Nachteilen zu beginnen, bricht mit den ungeschriebenen Regeln des Coming-out-Diskurses, ermutigend $\mathrm{zu}$ sein. Ziemlich diffus und in der Du-Form werden im Folgenden die Nachteile aufgezählt. Durch die Verwendung der Du-Form werden die Leser*innen direkt als potentiell von den Problemen des Post-Outing Betroffene angerufen. Fast schon dramatisch wird den Leser*innen ihr zukünftiges Schicksal vor Augen geführt. Die Aneinanderreihung wirkt diffus, weil sie erhebliche Leerstellen im Argumentationszusammenhang aufweist. Zu Beginn und zum Ende dieser Aneinanderreihung wird darauf vorbereitet, dass alles, was mensch macht, nach dem Coming-out anders gelesen wird: »Jeder Schritt den du tust, jedes Wort das du sagst, jeder Blick den du riskierst wird plötzlich anders gedeutet."Diese Reaktion der anderen auf das Coming-out wird als natürlich erklärt: »Menschen mögen keine Veränderung«. Von der naturalisierenden Aussage, dass Menschen keine Veränderungen mögen, wird auf die vorher beschriebene Reaktion auf das Coming-out geschlossen. Was bleibt, ist eine Lücke in der Argumentation: Es wird nicht erklärt, wie beides miteinander zusammenhängt.

Gleichzeitig wird der Akt des Coming-out in dieser Argumentation gleich mehrfach bestimmt: »Veränderst du etwas, indem du etwas noch nie Dagewesenes aussprich[s]t, einen Teil von dir offenbarst oder auch nur versuchst, deine wahrscheinlich liebsten Menschen an deinem Leben voll und ganz teilhaben zu lassen, dann riskierst du leider [...].« Das Coming-out wird hier als ein Sprechen bestimmt, das Veränderungen be- 
wirkt und risikobehaftet ist. Es wird als eine »Offenbarung«, ein »Wahrsprechen« über sich selbst festgeschrieben, aber auch als etwas, durch das die Familienbeziehung auf eine bestimmte Art und Weise neu hergestellt wird (»deine liebsten Menschen [...] voll und ganz teilhaben lassen«). In der ersten Bestimmung wird das Coming-out als ein Sprechakt, der verändert, festgeschrieben. Im Anschluss an Judith Butler kann insofern von einem performativen Akt gesprochen werden, als durch das Sprechen etwas hergestellt wird. Gleichzeitig wird der Sprechakt aber auch als ein Akt markiert, in dem »nur immer schon Dagewesenes « ausgesprochen wird. Damit wird in der Art und Weise, wie das Coming-out als Sprechakt festgeschrieben wird, verkannt, dass sich das sprechende Subjekt erst im Sprechen als das herstellt, was es (vermeintlich) immer schon gewesen ist. Das Coming-out wird durch die Begriffe der »Offenbarung« oder des endlich »voll und ganz teilhaben«-Lassens auch als ein Modus des »Wahrsprechens über sich selbst « bestimmt. Mit dem Begriff der »Offenbarung« - wie auch dem Begriff des "Bekennens ${ }^{186}$ - wird eine christliche Terminologie bemüht. Hier zeigt sich, was Foucault als Charakteristikum des christlichen Abendlands beschrieben hat, nämlich wie in Form eines Geständnisses über die eigene Sexualität eine Beziehung zwischen Subjektivität und Wahrheit hergestellt wird:

»Bei der Sexualität wurde der wahre Diskurs, zumindest zu einem beträchtlichen Teil, als obligatorischer Diskurs des Subjekts über sich selbst institutionalisiert. Das heißt, er wird nicht ausgehend von etwas aufgebaut, was sich als Beobachtung und Prüfung ausgibt, nach Regeln, denen Objektivität zugestanden wird, vielmehr wird der wahre Diskurs über die Sexualität rund um die Praktik des Ceständnisses ausgebaut. ${ }^{187}$

Das Coming-out als Sprechen über das, was man schon immer gewesen ist, wird zudem als ein risikobehafteter Akt, durch den mensch sich verletzbar macht, beschrieben. Die Reaktion der anderen, die etwa darin besteht, »dich falsch oder anders einzuschätzen «, beschreibt das Risiko des Coming-out. Es ist verletzend, wenn der Versuch, den liebsten Menschen noch näher sein zu wollen, scheitert. In der vorgetragenen Dramaturgie dieser Aussage schreiben sich auch die von der Autor*in persönlich erfahrenen Verletzungen ein. All diese mehrfachen Bestimmungen dessen, was das Coming-out ist, sind in den folgenden Abschnitten präsent. Um sie herum ordnet sich das, was gesagt wird, beziehungsweise das, was sagbar oder nicht sagbar ist, an.

Im zweiten, sehr kurzen Abschnitt schreibt die Autor*in, dass ihre Reaktion im Nachhinein betrachtet etwas überempfindlich gewesen sei. Auch die »Veränderung« durch das Coming-out wird differenzierter betrachtet: Es sind die »Bilder in den Köpfen der Mitmenschen«, die sich verändern.

$\mathrm{Zu}$ Beginn werden in diesem, der Einleitung folgenden Abschnitt die verallgemeinerten vorherigen Aussagen als ein eigentliches Sprechen über die eigenen Erfahrungen offengelegt: "Jedenfalls habe ich genau das empfunden.« Die Verletzlichkeit, die sich durch diese Dramaturgie in den Text eingeschrieben hat, wird im Anschluss relativiert. Die Autor*in schreibt, dass sie im Nachhinein weiß, dass sie »überempfindlich war«

186 Der Ausdruck »sich zu sich selbst bekennen« wird auf der Homepage verwendet.

187 M. Foucault: Subjektivität und Wahrheit, S. 31. 
und »einfach nur Gespenster gesehen hat «. Die eigenen erfahrenen/empfundenen Verletzungen werden heruntergespielt. Mit der verbreiteten Redewendung des »Gespenstersehens « wird nahegelegt, dass die Autor" in etwas empfunden habe, was gar nicht da war. Die affektiven Reaktionen auf die »Post-Outing «-Erlebnisse, die offensichtlich real empfunden wurden, werden als »falsche« oder »übertriebene« Bewertung einer Situation beschrieben. Ihnen wird das »Wahrsprechen« abgesprochen.

Danach wird die Bestimmung des Coming-out als ein Sprechakt, der verändert, erneut aufgegriffen: »In diesem Zusammenhang von Veränderung zu sprechen ist eigentlich nicht richtig. Eigentlich bist du nur ehrlich." Ein ehrlicher Sprechakt wird nicht als ein Sprechakt, der Veränderungen bewirkt, verstanden, weil er ja nur das, was wahr ist, ausspricht. Der folgende Satz widerspricht der Aussage, dass es nicht »richtig « sei, von Veränderung zu sprechen: »Trotzdem veränderst du feste Bilder in den Köpfen deiner Mitmenschen.« Dieser Widerspruch zeigt sich daran, dass das Coming-out auf der einen Seite als »wahrer Sprechakt festgeschrieben werden soll - der daher nicht verändern kann -, auf der anderen Seite aber die erfahrenen veränderten Reaktionen Thema des Artikels sind. Der Widerspruch äußert sich auch darin, dass die konstitutive Verwobenheit der sprechenden Person mit den Adressat*innen des Coming-out geleugnet wird, indem Veränderung als etwas beschrieben wird, das sich nur auf der einen Seite einstellt. Implizit steckt darin die Aussage, dass sich eigentlich nichts verändern sollte, wenn mensch einfach nur »ehrlich« ist. Die Diskrepanz zwischen dem "Eigentlich « und dem, was erlebt wurde, wird mit den festen »Bildern in den Köpfen der Menschen « begründet. Schuld an den Veränderungen - den erfahrenen Verletzungen - haben dieser Argumentation zufolge gesellschaftliche Normen und Stereotype. Der Akt des Coming-out wird dementgegen als »rein« beschrieben - er ist ehrlich. Auch in der Bestimmung des Coming-out als einen ehrlichen, authentischen Sprechakt zeigt sich, Foucault folgend, eine wesentliche Bestimmung abendländischer und christlicher Subjektivität: »Dieses Grundmodell der Subjektivität besteht [...] aus dem Vorhandensein einer Authentizität, einer tiefen Wahrheit, die es zu erkennen gilt und die den Untergrund, den Sockel, den Boden unserer Subjektivität bilden soll. ${ }^{188}$

Im dritten Abschnitt wird beispielhaft eine klassische familiäre Situation vor dem Outing beschrieben, bei der die Mitglieder einer Familie gemeinsam einen »Bond-Film« anschauen.

Wieder beginnt der Abschnitt mit dem Versuch, Distanz zum Geschriebenen zu wahren, indem erneut in der Du-Form geschrieben wird: »Nebenbei bemerkst du wie gut das Kleid von Bond-Girl Eva Green doch aussieht und wie cool James diesen nagelneuen BMW gerade wieder zu Schrott gefahren, hat ohne daß seine Krawatte verrutscht ist.«Danach wechselt der Text in die Ich-Form, es wird im Konjunktiv berichtet, wie die Situation »normal « weitergegangen wäre: Es wäre lebhaft über das Kleid des Bond-Girls diskutiert worden und am Wochenende wären sie gemeinsam shoppen und ins Kino gegangen. Es wird eine glückliche und idyllische Familiensituation beschrieben, die auch für die zuvor in der Du-Form angerufenen Leser*innen als gültig vorausgesetzt wird. Mit dem Begriff »normal« wird die Prä-Coming-out-Situation beschrieben, die bereits 
suggeriert, dass die Situation danach »nicht normal« sei. Die Beschreibung dieses Beispiels abschließend, wird über das Schweigen des Prä-Coming-out-Lebens gesprochen. Dieses Schweigen verweist auf einen Mangel, etwas, das in dieser glücklichen Familiensituation fehlt: »Natürlich hätte Mum sich auch noch über diesen ungeheuerlich heißen Typen ausgelassen, wozu ich vornehm geschwiegen hätte und mir einfach weiter das >Bond-Girk angesehen hätte ;-)«. Vor dem Coming-out ist das "glückliche«, »normale« Familienleben nur durch das »Schweigen« getrübt, das - im Gegensatz zum Comingout als ehrlichen Sprechakt - als etwas »Unehrliches« markiert wird.

Im vierten Abschnitt wird, im Vergleich zur Situation vorher, die »Reaktion nach dem Outing « beschrieben. Den Kommentaren zum »Bond-Film« folgen schräge Blicke, die Frage, ob »Lesben« immer so seien, und die Aufforderung, leise zu sein, damit alle den Film weiterschauen können. Die anerkennende Aussage über den BMW wird mit »Du bist seit neustem so burschikos!« kommentiert und der neue Klamottenstil wird als zu »unfeminin« diskutiert.

Im Vergleich zur vorher beschriebenen Familiensituation wird in diesem Abschnitt beispielhaft aufgezeigt, was in der Einleitung bereits angedeutet wurde (»[j]eder Schritt den du tust, jedes Wort das du sagst, jeder Blick den du riskierst wird plötzlich anders gedeutet«). Nachdem die Autor*in im Coming-out »offenbart«, wer sie ist, wird ihr gesamtes Handeln auf diese "Identität « reduziert. Abschließend wird in diesem Abschnitt gegen die Reaktionen der Eltern argumentiert, indem die von den Eltern hergestellte Beziehung zwischen der im Coming-out offengelegten sexuellen Orientierung einerseits und Kleidungs-, Sprech- und Verhaltensweisen andererseits in Frage gestellt wird: Die Eltern, so das Argument, hätten ihr bereits im Alter von drei Monaten blaue Nike-Schuhe gekauft. Der »burschikose« Kleidungsstil hängt, so das Argument, nicht zwingend und nicht nur mit der sexuellen Orientierung zusammen. Dieses fast schon affektive Nein zum Zusammenhang zwischen der »Sexualität« und als »burschikos« oder »unfeminin« geltenden Kleidungs- und Verhaltensweisen wird im Anschluss mit einem »Nun ja ich schweife ab« relativiert. In diese letzten Sätze schreibt sich ein, wie die Autor*in - durch das eigene Schreiben affiziert - (erneut) auf eine sich verteidigende Position zurückgeworfen wird.

Im fünften Abschnitt wird zusammengefasst, dass, basierend auf Vorurteilen, viele Dinge »anders « interpretiert worden seien, was das Leben erschwert habe: Auf einmal wird in der besten Freundin eine Partnerin gesehen und jedes längere Telefonat mit einem Mädchen als Hinweis auf eine Beziehung gewertet. Freundschaften zu Mädchen sind plötzlich undenkbar.

Zunächst einmal werden die »falschen« Zuschreibungen in doppelter Hinsicht als falsch ausgewiesen: Sie sind »durch Vorurteile über die Szene bedingt« und in Bezug auf die Autor*in falsch: »mir wurde plötzlich eine Beziehung angedichtet«. Die Erfahrung, ständig mit falschen Zuschreibungen konfrontiert zu werden, wird als belastend beschrieben: »was mir das Leben nicht unbedingt einfacher machte«. Diese Erzählung widerspricht der Relativierung der erlebten Verletzungen aus dem zweiten Abschnitt. Offensichtlich hat die Autor"in nicht nur "Gespenster gesehen«. Auch in diesem Abschnitt wird im abschließenden Satz die affizierende Wirkung des Sprechens über die- 
se Situationen spürbar, wenn die Autorin beinahe wütend fragt, ob man denn nicht auch einfach mit Mädchen befreundet sein könne: »War es denn plötzlich undenkbar, mit Mädels nur noch befreundet zu sein?«

Im sechsten, sehr kurzen Abschnitt wird Kommunikation als der Weg beschrieben, um auf die durch das Coming-out hervorgerufenen Veränderungen in der Familie einzugehen. Zunächst wird der Familie Zeit eingeräumt und auf eine notwendige vertraute Basis verwiesen, "gerade, weil ein Outing für Familienmitglieder eine Veränderung bedeutet«.

Die im zweiten Abschnitt festgeschriebene Verleugnung der konstitutiven Verbundenheit mit den anderen (der Familie), die eine Veränderung nur auf Seite der Familie und nicht auch auf Seite des sprechenden Subjekts verortet, schreibt sich in diesem Abschnitt lückenlos fort. Es ist die Familie, die Zeit braucht, und es ist die den Sprechakt des Coming-out ausführende Person, die eine vertraute Basis herzustellen hat: »Der Schlüssel, den ich für mich fand war Kommunikation. "Die Verantwortung, den »Schlüssel« zu finden, um das zerstörte Familienglück wiederherzustellen, wird der die Veränderung »initiierenden« Person zugeschrieben. Die Bürde der Verantwortung für negative Konsequenzen durch den Bruch gesellschaftlicher Normen wird denjenigen, die nicht der Norm entsprechen, auferlegt: »the work we do when we aim to transform the norms of an institution, and the work we do when we do not quite inhabit those norms. These two senses often meet in a body: those who do not quite inhabit the norms of the institution are often those given the task of transforming these norms. $"{ }^{189}$ Es ist die Anerkennung der dem Akt des Coming-out vorausgehenden Schuld, die sich in die so hergestellte Notwendigkeit, als outendes Subjekt den Schlüssel zu finden, einschreibt. Aufgrund dieser Schuld tragen die Jugendlichen die Verantwortung für das, was in der Familie zerbrochen ist, und für die erfahrenen Verletzungen. Sie müssen gegen beides arbeiten.

Im siebten Abschnitt wird beschrieben, wie durch Kommunikation wieder Normalität hergestellt werden könne. Obwohl es nervenaufreibend gewesen sei, habe sie, die Autor" in, immer wieder erklärt, dass sie schon immer gerne Chucks getragen habe, dass Lesben Männer nicht hassen würden, dass nicht alle kurze Haare hätten, dass es auch möglich sei, eine Familie zu gründen, und dass Lesbischsein keine Phase sei.

Der »Schlüssel« - die Kommunikation - auf dem Weg zurück zur Familienidylle wird als nervenaufreibend beschrieben: "Auch wenn es mir irgendwann tierisch auf die Nerven ging, war es nötig meiner Mutter auch 5-mal in einer Woche zu erklären, dass [...].« Es wird doppelte emotionale Arbeit geleistet: Die erfahrenen Verletzungen werden ertragen und die Arbeit an der Institution Familie wird als nervenaufreibend empfunden. Das Leben nach dem Coming-out wird, ähnlich wie Ahmed es für ein feministisches Leben beschreibt, als ein Versuch beschrieben, die Dinge am Laufen zu halten: »We can be shattered by what we come up against. And then we come up against it again. We can be exhausted by what we come up against. And then we come up against it 
again. ${ }^{190}$ Die zirkuläre Wiederholung, die darin besteht, sich immer wieder gegen das zu wenden, was sich gegen dich wendet, kann, auch Ahmed zufolge, kraftraubend und auslaugend sein. »No wonder: we might feel depleted. [...] By referring to >feeling depleted ‘, I am addressing a material as well as embodied phenomenon: of not having the energy to keep going in the face of what you come up against. « ${ }^{191}$ Die Schuld für das, was durch das Coming-out zerbrochen ist - die normale glückliche Familienbeziehung wird abgetragen, indem diese Verletzungen ausgehalten werden und immer wieder an dem gearbeitet wird, was gegen einen selbst arbeitet. In dem Abschnitt werden die auf Vorurteilen basierenden verletzenden Aussagen (»we can be shattered«), gegen die die Autor*in trotzdem immer anredet (»we come up against it again«), aufgezählt und als »nervenaufreibend « beschrieben (»we can be exhausted by what we come up against «). Zusätzlich zu den falschen Zuschreibungen (»Lesben hassen Männer«, »haben kurze Haare «) wird die Autor* in mit der Sorge, dass »Mama« nicht »Omi« werden kann, konfrontiert. Sie arbeitet gegen das, was ihre Eltern unglücklich macht (»Mama kann nicht Omi werden«), weil aufgrund des Coming-out der für sie vorgesehene »Path of Happiness ${ }^{192}$ in Gefahr ist. Es sind Vorurteile, falsche Interpretationen, die Sorge über die verlorene Möglichkeit des familiären Glücks, aber auch die Angst, nicht ernst genommen $\mathrm{zu}$ werden (das ist sicher nur eine Phase), gegen die die sich outende Person $\mathrm{zu}$ arbeiten hat. Den Schlüssel zur »Normalität« einzusetzen, bedeutet, die Schuld abzutragen, indem Verletzungen und nervenaufreibende Arbeit allein auf das sich outende Subjekt gelegt werden.

Im achten Abschnitt wird betont, dass es wichtig gewesen sei, immer wieder aufzuzeigen, dass die Autor*in sich als Person infolge des Coming-out nicht verändert hat. Der Vorteil des zu Hause Wohnens sei, dass die Familie zwangsläufig immer wieder mit der Homosexualität der Autor*in konfrontiert wird und sich so daran gewöhnen kann. Auch für die Autor*in gab es neue Ereignisse: »die erste Freundin« mit nach Hause bringen oder der »erste Liebeskummer«. Die Familie konnte mit der Zeit Vorurteile abbauen, die Szene kennenlernen und sich ein eigenes Bild machen.

Zusammenfassend wird betont, dass es in der Arbeit an der Familie zentral gewesen sei, immer wieder klarzumachen, dass das Lesbischsein eben nicht die ganze Person durchdringt und verändert. Arbeit an der Familie bedeutet, die Verknüpfung zwischen der im Coming-out ausgerufenen Identität und dem, was mensch als Person ist, zu entkoppeln. Es geht darum, der Familie zu zeigen, dass sich mit dem Coming-out auf Seite des sprechenden Subjekts nichts verändert. Im Weiteren wird nun auf den Vorteil des zu Hause Wohnens nach dem Coming-out eingegangen: »Der Vorteil des Zuhause Wohnens war hier ganz klar, dass jedes Familienmitglied jeden Tag aufs Neue mit der Situation konfrontiert wurde und so zwangsläufig einen Weg finden musste damit auf seine_ihre ganz eigene Art und Weise klarzukommen.« Dadurch, dass es kein Entkommen gibt, muss der nervenaufreibende Kampf zwangsläufig geführt werden. Es ist ein Vorteil, weil und solange dieser Kampf Erfolg verspricht: »Nach und nach wurden die 
Klischees abgelöst. Jede_r machte sich mit der Zeit sein_ihr eigenes Bild von dem, was es nun heißen würde so zu leben wie ich.« Indem zudem berichtet wird, dass auch für die geoutete Person viele »neue Ereignisse« hinzukamen, wird der zu Beginn des Artikels getätigten Aussage widersprochen, dass sich bei der Person, die sich outet, nichts ändern würde. »Mein erstes Mädchen mit nach Hause bringen«, »mich durch meinen ersten richtigen Liebeskummer [...] zu trösten«, »die neusten Aktionen im örtlichen Düsseldorfer Schwul-lesbischen Jugendzentrum PULS zu verfolgen«. Die Vorteile des Coming-out zu Hause bestünden zum einen darin, mit der Notwendigkeit konfrontiert $\mathrm{zu}$ sein, die Normalität wiederherzustellen, zum anderen darin, die Möglichkeiten zu haben, die eigenen Erfahrungen mit der Familie zu teilen.

Im kurzen neunten Abschnitt wird resümiert, dass sich der »Konflikt« um einiges länger gezogen hätte und entsprechend frustrierender gewesen wäre, hätte die Autor*in nicht mehr zu Hause gelebt.

Der Zeitfaktor und die unausweichliche Konfrontation machen, dieser Argumentation zufolge, einen relevanten Unterschied: Wenn mensch noch zu Hause wohnt, kann der »Konflikt« schneller gelöst werden. Entsprechend sei es weniger frustrierend.

Im zehnten Abschnitt erzählt die Autor*in, dass sie nach einigen Monaten wieder ein "ganz normales Familienmitglied « gewesen sei und dass ihre Aussagen über das »BondGirl« von da an mit peinlichen Sprüchen wie »das ist also dein Typ?« kommentiert worden seien.

Nach einigen Monaten ist die »Normalität« - die Familienidylle - wiederhergestellt. Mit dem Unterschied, dass das »Schweigen« gebrochen ist und die Autor*in ihre Familie jetzt voll und ganz teilhaben lassen kann. Der Vater kommentiert die Aussage über das »Bond-Girl« jetzt mit: »Ahh! Also das ist dein Typ. Ich muss sagen mein Töchterchen hat Geschmack«. Die wiederhergestellte Normalität schreibt sich auch darin ein, dass die Autorin - wie jeder »Teenager« - den Kommentar des Vaters peinlich findet: "So ein Kommentar von seinen Eltern ist und bleibt peinlich." Das Coming-out hat ein glückliches Ende genommen, die »normale Familienidylle« ist wiederhergestellt, diesmal ohne Schweigen.

Im elften Abschnitt wird zusammenfassend dargelegt, dass das zu Hause Wohnen und die damit einhergehende zwangsläufige Konfrontation die Rückkehr in den normalen Alltag möglich gemacht hätten und die Familie dadurch sogar ein wenig toleranter geworden sei.

Zunächst stellt die Autor*in sich als nunmehr »aktiv« und »bewusst « handelndes Subjekt her und betont »[d]ie Tatsache, dass ich mich outete während ich zu Hause wohnte«. Hier klingt eine aktive bewusste Entscheidung für die Konfrontation statt einer erzwungenen Konfrontation an. Die Normalität - »ein ruhiges und harmonisches Alltagsleben mit meiner Familie« - und die Akzeptanz dessen, was die "geoutete« Person ist, markieren das Happy End der Post-Coming-out-Story. Darüber hinaus hat das Coming-out - auch hier ist die Autor"in aktives Subjekt - es auch möglich gemacht, positive Veränderungen zu bewirken, indem die Familie toleranter geworden sei: »Themen wie Toleranz, Akzeptanz und Homosexualität [...] sind in einer doch eher konser- 
vativ geprägten Familie Alltagsthemen geworden.« Die Autor*in stellt sich im letzten Abschnitt als aktiv handelndes Subjekt her, das insofern handlungsmächtig ist, als die Post-Coming-out-Konflikte zu einem Happy End gebracht wurden und die Familie sich sogar weiterentwickelt hat - sie ist toleranter geworden. Vergessen sind die Verletzungen und die nervenaufreibende Arbeit.

Im abschließenden Abschnitt wird resümiert, dass das Lesbischsein zu Hause weder Nachteile noch Sonderrechte bringt. Nach viel Auseinandersetzung sei es etwas ganz Normales geworden.

$\mathrm{Zu}$ Beginn dieses Abschnitts steht in Anführungszeichen der Satz: »Ich bin Kimi und ich stehe auf Frauen.« Dieser Satz ist ein möglicher Coming-out-Satz. Dass sie Kimi ist und auf Frauen steht, ist »nach einem intensiven Prozess mit viel Arbeiten von allen Beteiligten eine ganz normale Sache geworden«. »Ich bin Kimi und ich bin lesbisch« ist nicht nur der Anfang - der Sprechakt, durch den das Coming-out zu einem das Leben anordnenden Ereignis wird -, sondern es ist auch der Endpunkt. Es ist Normalität geworden, dass sie Kimi ist und auf Frauen steht. Es ist eine Normalität, die hart erkämpft ist. Entsprechend ist es auch ein stolzer und ermächtigender Ausruf: »Ich bin Kimi und ich stehe auf Frauen.«

\section{Doppelte Arbeit}

Die »Out!« als Zeitschrift von queeren Jugendlichen für queere Jugendliche ist ein Ort, an dem ein Austausch über eigene Coming-out-Erfahrungen stattfindet. Das Schreiben über die Coming-out-Erfahrungen ist eine Möglichkeit, die eigenen Erfahrungen zu verarbeiten und andere auf das vorzubereiten, was auf sie zukommen könnte. Es stellt aber auch insofern einen Zusammenhalt her, als es eine spezielle gemeinsam geteilte Erfahrung gibt. Im Sprechen über das Coming-out wird ein spezifisches Wir hergestellt, indem das Coming-out auf eine bestimmte Art und Weise denkbar gemacht wird. In der »Out! « wird das Coming-out zunächst im Sinne einer christlichen Geständnispraxis als ein ehrlicher Akt der »Offenbarung« sagbar. Die Jugendlichen »bekennen« im Coming-out, wer sie immer schon gewesen sind. Diesem Geständnis geht eine Schuld voraus. Diese Schuld wird zunächst den "festen Bildern in den Köpfen der Menschen« zugeschrieben, die keine Veränderung mögen. Die Schuld wird dann jedoch in das die Veränderung auslösende sprechende Subjekt verlegt, indem die Verantwortung für das, was in der Familie zerbrochen ist, übernommen wird. Durch die beschriebene gleichzeitige Arbeit an den erfahrenen Verletzungen und an der Institution Familie - beides emotional belastend - wird die Schuld getilgt. Im Sprechen über das Coming-out wird eine Notwendigkeit festgeschrieben, die verletzenden Erfahrungen auszuhalten und die Verantwortung dafür zu übernehmen, die zerbrochene Familienidylle wiederherzustellen. Vorausgesetzt wird, dass sich die Normalität in der Familie wieder einstellt und dass das sich outende Subjekt sich letztendlich als stolzes und handlungsmächtiges erfahren kann. 


\subsubsection{Der Weg zum Glück}

Auch im »Milchbüechli« spielt das Coming-out eine wichtige Rolle. Im Analysezeitraum gibt es allerdings keine eigene Coming-out-Rubrik. ${ }^{193}$ Generell ist diese Zeitschrift nicht so grundlegend um das Coming-out angeordnet wie die »Out!«. Das Thema kommt aber trotzdem in fast jeder Ausgabe mindestens in einem Artikel vor, oft sogar in mehreren Artikeln. Zum einen, weil Jugendtreffs und Projekte vorgestellt werden, die um das Coming-out angeordnet sind und dank derer die Milchjugend als Netz, das all diese Gruppen zusammenhält, funktioniert, zum anderen, weil die »vielen Schreiberlinge «ihre Gedanken, Meinungen und Wünsche präsentieren« und die Milchjugend sich eben auch Gedanken zum Thema Coming-out macht. ${ }^{194} \mathrm{Im}$ »Milchbüechli« werden entsprechend unterschiedliche Coming-out-Geschichten erzählt: vom Trans*-Comingout über das "afrikanische« Coming-out und das unfreiwillige Coming-out bei den Großeltern bis hin zu Coming-out-Geschichten in der Rubrik »Der Jugend erste Male«. Auch in der Rubrik »Fragestunde« werden Fragen zum Coming-out beantwortet. Dabei gehören die Erzählungen zum Coming-out zu den wenigen Erzählungen im »Milchbüechli«, die nicht durchgehend positiv sind - am Ende aber immer gut ausgehen. Es wird von der Community als unterstützende Ressource im und für das Coming-out gesprochen. Zudem wird die Sichtbarkeit als politische Notwendigkeit, die ein Comingout erfordert, thematisiert. Auch der Slogan, in dem und durch den die Milchjugend sich häufig auf Demonstrationen repräsentiert - »Die beste Phase meines Lebens« nimmt Bezug darauf, dass viele Jugendliche in ihrem Coming-out nicht ernst genommen werden und dieses als »Phase« abgetan wird. Die Milchjugend engagierte sich 2015 auch mit einem Stand am Internationalen Coming Out Day mit einer »Ich oute mich als«-Aktion, an der Menschen sich als »Seegurke, Freak, Couch Potato oder ähnliches geoutet haben «. ${ }^{195}$ Hier wird das Coming-out auf alltägliche Dinge ausgeweitet und von der engen Verknüpfung mit der sexuellen Orientierung entkoppelt. Die Milchjugend selbst ist eine Gruppe, die ihre "Falschheit « stolz und sichtbar in die Öffentlichkeit trägt und entsprechend das Coming-out auch als politische Strategie versteht: "Die Menschheit braucht Coming-Outs noch. [...] Je mehr, desto besser, denn je mehr Menschen in Berührung mit queeren Identitäten kommen, desto schneller schwinden die Berührungsängste und Hemmungen, die mensch gegenüber Falschsexuellen noch haben könnte.«196

In der Ausgabe drei des »Milchbüechli« mit dem Thema »drei und mehr«, die im März 2013 erschienen ist, ist das Thema Coming-out besonders präsent. ${ }^{197}$ Es wird über - unter anderem um das Coming-out angeordnete - Jugend- und Student*innengruppen geschrieben, über die Schwierigkeiten des Coming-out in der Berufsschule berichtet, es wird vom Coming-out bei den Großeltern erzählt und es gibt Hinweise und

Seit 2019 wird das »Milchbüechli« allerdings auf der Website auch damit beworben, dass es in jeder Ausgabe eine Coming-out-Story gibt.

Ebd.

Milchjugend: drei und mehr. 
Tipps, wie der erste Freund mit nach Hause gebracht werden kann. Letzterer ist auch der für die Feinanalyse ausgewählte Artikel, dessen Argumentationsverlauf im Folgenden näher dargestellt werden soll. ${ }^{198}$ Der Artikel hat die Überschrift »DAS IST ER! «. 199 Das in der Überschrift ausgerufene »DAS IST ER! ${ }^{200}$ bezieht sich auf den Sprechakt, durch den der Freund bei den Eltern vorgestellt wird. - Hier! »Das ist er « - mein neuer Freund. Die Unterüberschrift ist im Stil eines Klappentexts geschrieben:»Endlich habe ich meinen ersten Freund. Doch jetzt stellt sich die Frage: Kann ich den nach Hause bringen? Eine kleine Wegbegleitung«. Indem in der Ich-Form geschrieben wird, wird eine persönliche Nähe zu allen Leser*innen, die diese Erfahrungen (zukünftig) teilen, hergestellt. »Endlich habe ich meinen ersten Freund.« Ähnlich wie im »Out!«-Artikel wird auch hier zunächst das Sprechen über das Coming-out kontextualisiert. Es geht um die Situation, dass der erste Freund mit nach Hause gebracht werden soll: »Doch jetzt stellt sich die Frage: Kann ich den nach Hause bringen?« Auch hier führen ähnliche Aspekte wie im »Out!«-Text zu einer spezifischen Situation, die das Coming-out rahmen und ebenfalls unbenannt bleiben: zum einen die Familiensituation als eine, in der die Erfahrung, von der Norm abzuweichen, oft nicht geteilt wird und eine normative Identität vorausgesetzt wird, zum anderen die »ungewählte« gemeinsame Wohnsituation. Im Klappentext wird auch der Zweck/das Ziel des Artikels festgeschrieben. Der Artikel hat den Anspruch, die Leser*innen auf diesem »Weg« zu »begleiten«. Der Artikel will also eine Wegbegleitung sein. Die Wegbegleitung, die eng mit der Frage nach dem Coming-out verknüpft ist, ist in sechs Abschnitte unterteilt.

Im ersten Abschnitt werden die Schritte vom Kennenlernen bis zum Paar aufgezählt. Der nächste Schritt ist, den Freund mit ins eigene Zimmer zu nehmen. Die »Hürde«, die es dabei zu bewältigen gilt, seien die Eltern.

In diesem ersten Abschnitt wird nochmals in die Situation und das Problem, um das es im Artikel geht, eingeführt. Mit drei kurzen Sätzen wird der Weg zur Partnerschaft beschrieben: "Die ersten Treffen sind gut gegangen. Der Neue ist dir immer sympathischer geworden. Inzwischen seid ihr ein Paar.« Das ist der Punkt, an dem die Wegbegleitung ansetzt. Der nächste wichtige Schritt in der Beziehung: »ihn noch mehr in dein Leben ein[zu]bauen«, indem du ihm »zeigst«, wie du »lebst«. Dem neuen Freund das eigene Zimmer zu zeigen, wird in mehrfacher Hinsicht als notwendiger nächster Schritt auf dem Weg zu einer glücklichen Beziehung festgeschrieben: Es ist zum einen ein Vertrauensbeweis: »Der Einblick ins eigene Heim oder Zimmer ist einer in deinen privatesten Bereich «. Zum anderen ist das Zimmer ein für die Beziehung wichtiger zukünftiger Rückzugsort: »Hier wird gemeinsam geträumt, geliebt, gelacht, geweint und geredet«. Um diesen Schritt auf dem Weg zur guten Beziehung, auf dem

198 Der Artikel wurde von Juri geschrieben, einem 21-jährigen Studenten aus Basel - so die Autor*innenbeschreibung. Juri schreibt eine Zeitlang häufiger für das »Milchbüechli«, ist aber keine derart zentrale Figur wie die Autor*innen der anderen näher vorgestellten Artikel. Homosexualität angerufen wird, kommt in den ersten Ausgaben - je nach Autor*in - häufiger vor. In späteren Ausgaben werden die »Anrufungen« offener.

200 Milchjugend: »DAS IST ER!«, in: drei und mehr, Milchbüechli 2013. 
der Text begleiten will, machen zu können, muss aber die »Hürde« Eltern überwunden werden. Die Eltern werden als ein »Hindernis« auf dem »Weg« zur glücklichen Beziehung bezeichnet, das es zu überwinden gilt. Damit ist das Problem, um das sich der Artikel anordnet, eingeführt: »Wie bringe ich ihn also mit nach Hause?« Es wird ein bestimmter Weg zum Glück festgeschrieben: den Freund mit nach Hause zu nehmen. Das Coming-out wird notwendig zum Thema, weil es die Hürde Eltern gibt.

Im zweiten Abschnitt wird zunächst erklärt, dass das Coming-out auch gut laufen könne und dass es keinen perfekten Plan geben könne. Der Artikel versucht dennoch, einige Gedanken und Erfahrungen zu teilen, die hilfreich sein könnten.

Im zweiten Abschnitt wird die Art und das Ziel der Wegbegleitung näher bestimmt. Zunächst einmal wird aber festgestellt, dass nicht alle diese Wegbegleitung bräuchten: »Vielleicht klappt bei dir alles bestens«. Indem die Zielgruppe eingeschränkt wird, wird an die vermeintliche Notwendigkeit eines ermutigenden Coming-out-Diskurses angeknüpft. Anders als im »Out!«-Artikel, in dem alle sich outenden Jugendlichen mit ihrem zukünftigen Schicksal konfrontiert werden (alles, was du tust, wird plötzlich anders gedeutet), werden hier unnötige Sorgen und Ängsten genommen. Gleichzeitig wird die Zielgruppe (»wenn ihr in der beschriebenen Lage seid, und nicht so recht wisst, wie vorgehen«) und die Art der Wegbegleitung beschrieben (»[i]ch will hier keine Anleitung schreiben, wir ihr es am besten machen könnt [...]. Aber ein paar Erfahrungen, Ideen und Gedanken sollen euch helfen«). Abschließend wird in Bezug auf das Comingout die Notwendigkeit der Wegbegleitung begründet: »Wenn du geoutet bist, ist alles einiges leichter. Wissen deine Eltern nicht, dass du schwul bist, musst du Tricks anwenden. " Das Coming-out wird als der Moment festgeschrieben, der den Unterschied macht, der darüber entscheidet, wie kompliziert es ist, den nächsten wichtigen Schritt auf dem Beziehungsweg einzuschlagen. Die Leser*innen, die begleitet werden, werden in der Du-Form angesprochen. Dadurch wird eine bestimmte Nähe erzeugt, erinnert diese Form des "Sprechens « doch an eine direkte Interaktion mit Freund"innen oder Bekannten, von denen mensch Tipps bekommt.

Die nächsten drei Abschnitte sind im Gegensatz zu den anderen Abschnitten formal nur durch einen Absatz, nicht durch eine Leerzeile markiert, was auch ihrer inhaltlichen Verknüpfung entspricht. Es werden drei verschiedene Szenarien aufgezeigt, »den Freund mit nach Hause zu nehmen«: »[w]enn du ungeoutet bist und das vorläufig auch so bleiben soll«, »[w]enn du ungeoutet bist, einen Freund hast und dich outen willst « und »[w]enn du einen Freund hast und geoutet bist«. In diesem Abschnitt wird das Coming-out - konkret die Frage, ob mensch "geoutet ist « und ob mensch »sich outen will« - als etwas, das einen Unterschied macht, festgeschrieben.

Im ersten Teil wird das erste Szenario - nicht "geoutet « $\mathrm{zu}$ sein und sich auch nicht outen $\mathrm{zu}$ wollen - durchgegangen. In diesem Fall wird die Möglichkeit der »beste[n] und häufigste[n] Ausrede« aufgemacht: Der Freund sei »der Kollege von der Schule«. Diese »beste« Möglichkeit wird allerdings im Anschluss gleich relativiert: »Nur würde `der Kollege ‘ vielleicht nicht jedes Wochenende bei dir übernachten. Und vor allem habt ihr nie richtig Zeit für euch.« Das, was zu Beginn als der nächste wichtige und richti- 
ge Schritt in der Beziehung beschrieben wird, kann - das suggerieren die aufgezählten Nachteile - in diesem Szenario nicht zufriedenstellend umgesetzt werden. Ein weiterer Nachteil sei, dass die Eltern nicht »blöd« sind und wissen, dass Jugendliche versuchen, »die eigenen Eltern zu umgehen«. Hier wird implizit eine moralische Komponente angesprochen. Die Ausrede mit dem »Kollegen« ist eine Art, die »Eltern zu umgehen«, ein »Versteckspiel«, also auch eine Art des Lügens. Das - so das Fazit dieses Abschnitts ist »wohl keine Lösung auf Dauer«. Das Szenario, nicht geoutet zu sein und sich auch nicht outen zu wollen, wird als Möglichkeit aufgemacht, um gleich wieder verworfen zu werden. Es ist hinderlich für die Beziehung und es ist unehrlich gegenüber den Eltern.

Als Nächstes wird das Szenario, das sich ergibt, »wenn du ungeoutet bist, einen Freund hast und dich outen willst«, besprochen. Vor dem Coming-out könnte der Freund beispielsweise als »Kumpel« schon mal mit nach Hause genommen worden sein oder er ist vielleicht auch schon öfter zu Besuch gewesen. In diesem Fall kennen die Eltern den Freund zum Zeitpunkt des Coming-out bereits. Sollten die Eltern den Freund noch nicht kennen, wird empfohlen ihn nicht gleich beim Coming-out zu erwähnen.

Zunächst einmal werden verschiedene Möglichkeiten genannt, wie der Freund vor dem Coming-out mit nach Hause genommen werden könnte - Möglichkeiten, bei denen die Eltern den Freund bereits vor dem Coming-out mehr oder weniger intensiv kennenlernen können (»[e]r war schon mal bei dir, als du sturmfrei hattest «, »[d]u hast ihn schon mal als >Kollegen < [...] vorgestellt « und »[e]r ist relativ oft bei dir, die Eltern fragen aber nicht«). Mit Ausnahme der zweiten Option wurden diese Möglichkeiten im ersten Szenario (nicht geoutet sein und sich auch nicht outen wollen) nicht eröffnet. Offen bleibt, warum diese Optionen nur als Möglichkeit benannt werden, wenn der nächste Schritt - das Coming-out - folgt: »Irgendwann outest du dich«. Das Comingout wird nüchtern als logisch folgender Schritt beschrieben. In Klammern wird allerdings darauf verwiesen, dass das Thema einen eigenen Artikel erfordern würde. Die Abfolge scheint jedoch klar festgeschrieben: Der Freund war auf die eine oder andere Art und Weise schon mal bei dir zu Hause und irgendwann outest du dich. Dass der Freund vorher nicht bei dir zu Hause war oder dass das mit dem Outing, obwohl du es willst, aus irgendeinem Grund doch nicht stattfindet, ist keine Option. Vom Punkt des Outings läuft es nahtlos weiter: »Im weiteren Gespräch kommt dann vielleicht die Frage, ob du einen Freund hast.« Dem »Irgendwann outest du dich« folgt in dieser Erzählung fast logisch die Frage nach dem Freund. Andere mögliche Reaktionen auf das Coming-out werden nicht genannt. Mit dem Freund sollten die Eltern - so der Text weiter - vielleicht nicht gleich auch noch »überrumpelt werden«: »Ich würde sie aber eher nicht zur selben Zeit auch noch mit einem Freund überrumpeln«. Nicht nur das Coming-out, auch die Tatsache, dass man tatsächlich in einer Beziehung ist - in der sich das, als was mensch sich »outet«, materialisiert -, wird als weitere, zusätzliche Überforderung für die Eltern dargestellt. Den Jugendlichen wird empfohlen, Rücksicht auf die Eltern zu nehmen. Das Prä-Coming-out-Szenario, das in der anfänglichen Erzählstruktur bereits gesetzt wird, nämlich, dass die Eltern den Freund schon kennen, weil er schon bei ihnen zu Hause war, wird als ein Aspekt beschrieben, der die Hürde, den Freund vorzustellen, leichter nehmen lässt: "Einfacher wird es vielleicht sein, weil sie deinen Freund ja schon kennen«. Abschließend wird noch einmal empfohlen, den 
Freund nicht gleich nach dem ersten Date mit nach Hause zu nehmen: »bring ihn nicht grad nach dem ersten Date heim«.

In diesem zweiten Szenario wird die Version, den Freund vorher schon einmal mit nach Hause genommen zu haben und ihn - den sie ja schon kennen - den Eltern ein paar Tage nach dem Coming-out vorzustellen, als die »leichteste « und auch als die einzig denkbare Option festgeschrieben. Die Wegbegleitung, die keine Anleitung sein will, gibt doch recht deutlich vor, wie das Outing und das Vorstellen des Freundes am besten funktionieren: ihn erst mit nach Hause bringen, sich dann outen. Das oberste Gebot scheint aber zu sein, Vorsicht walten zu lassen, wenn der Freund den Eltern vorgestellt wird (erst nach einiger Zeit und nicht gleich nach dem ersten Date).

Zuletzt wird das Szenario, das sich ergibt, »wenn du einen Freund hast und geoutet bist«, diskutiert. Nach dem Coming-out sollte der Freund nicht sofort mit nach Hause gebracht werden. Es solle lieber abgewartet werden, bis mensch eine Weile zusammen ist. Dann könne mensch den Freund zu einem gemeinsamen Abendessen oder Kaffee mit nach Hause nehmen. Es sei wichtig den Eltern Zeit zu geben, damit irgendwann alles ganz »normal« funktioniere.

Auch in diesem Szenario wird die Möglichkeit, dass die Eltern ihr Kind (auch) nach dem Coming-out akzeptieren, als einzige Option eröffnet: "Deine Eltern haben dich, wie es zu wünschen ist, so akzeptiert «. ${ }^{201}$ Im weiteren Verlauf legt die Wegbegleitung den Jugendlichen erneut nahe, trotzdem Vorsicht walten zu lassen, wenn sie den Freund mit nach Hause bringen wollen: »Erzähl von ihm, aber bring ihn nicht gleich in der ersten Woche mit, das wirkt etwas komisch, vor allem wenn die Beziehung dann doch nicht länger halten sollte.« Erst, wenn man sich gut »kennengelernt« hat und die Beziehung eine Weile anhält, wenn also eine sichere und glückliche Beziehung präsentiert werden kann - dann soll der Freund den Eltern vorgestellt werden. Die in diesem Abschnitt geforderte Rücksichtnahme ist ein Versuch, zu verhindern, dass ein homosexuelles Scheitern präsentiert wird. In Anlehnung an Sarah Ahmeds Ausführungen zum Coming-out, in denen dieses mit Fragen der Schuldigkeit und den Vorstellungen eines richtigen »Weges zum Glück« verknüpft wird, kann die Aussage als ein Versuch verstanden werden, zumindest ein Stück weit dem »Druck« zu entkommen, der das Verlassen des »richtigen «, heteronormativen Pfades mit sich bringt: der »Druck«, der auf denen lastet, die, wenn sie den Pfad verlassen, erst recht (den Eltern) beweisen müssen, dass sie glücklich sind: »Not to want your children to be unhappy can mean in translation: not to want them to deviate from the well-trodden paths. No wonder then that in some parental responses to a child coming out, this unhappiness is expressed not so much as being unhappy about the child being queer, but as being unhappy about the child being unhappy. « ${ }^{202}$ Die Erwartungen von Eltern an ihre Kinder sind geknüpft an das, was Ahmed »depth of happiness « nennt. Nach allem, was die Eltern für ihre Kinder getan haben, schulden diese ihnen wenigstens, "glücklich« zu sein.

201 Dass die Eltern das Coming-out akzeptieren, wird als allgemeingültige Aussage nur ein wenig relativiert, wenn ein »Wie es zu wünschen ist« eingefügt wird.

202 S. Ahmed: Living a Feminist Life, S. 51. 
Ähnlich wie in der »Out!« wird auch im »Milchbüechli« die mit dem Coming-out - der Bewegung in Richtung Identität - einhergehende Schuldigkeit anerkannt. Die sich outende Person macht sich schuldig, weil sie den Pfad des Glücks verlässt. Die Wegbegleitung legt den Jugendlichen nahe, diese Schuld einzulösen, indem sie versuchen, zu Hause zumindest keine scheiternde - sondern eine glückliche - Beziehung zu präsentieren. Auch darüber hinaus wird zur Rücksichtnahme gegenüber den Eltern aufgerufen: "Gib ihm und deinen Eltern Zeit, sich zu beschnuppern«. Die Eltern brauchen Zeit, sich daran zu gewöhnen, dass sich das, als was mensch sich »outet«, auch in tatsächlichen Beziehungen manifestiert. Gleichzeitig setzt dieses Szenario eine Familie voraus, in der sich die Eltern für das Beziehungsleben ihrer Kinder interessieren, sich Zeit zum Kaffeetrinken oder Abendessen nehmen und bei denen es entsprechend auch einen schlechten Eindruck macht, wenn die Beziehung nicht lange hält. Mit genügend Rücksichtnahme gegenüber den Eltern und ihren Erwartungen - so der Abschluss dieses Szenarios - »[k]ann er sicher auch mal bei dir übernachten« und wird ganz »normal« zu dir und »deiner Familie gehören«. Auch hier ist der Endpunkt der Erzählung ein glückliches Familienleben, in das Normalität einkehrt: Der Partner kann normaler Teil der Familie werden. Das Szenario, in dem mensch den Eltern "geoutet, langsam und rücksichtsvoll« den Freund vorstellt, wird gleichzeitig als das »beste« das Happy-End-Szenario - festgeschrieben.

Im sechsten Abschnitt wird noch einmal daran erinnert, dass »dieses Vorstellen« immer etwas komisch sei, aber kein Grund sei, nervös zu sein. Die Eltern müssten sich erst einmal an die Situation gewöhnen. Das Coming-out sei eine unangenehme Sache, durch die alle durchmüssten. Zudem könnten die Eltern den Partner*/die Partner*in unabhängig vom Geschlecht mögen oder nicht mögen.

Vorrangig ist im letzten Abschnitt, zum Coming-out zu ermutigen. Eine Strategie des Mutmachens ist eine »Am Ende wird alles gut«-Erzählung. Es gebe tatsächlich keinen Grund, nervös zu sein (»[a]ber nervös braucht deswegen keiner zu sein«), weil alles "gut gehen wird « und es nur eine Frage der Zeit sei, bis die Eltern sich daran »gewöhnen«. Das Coming-out wird des Weiteren als eine »unangenehme Sache«, durch die alle durchmüssen, »normalisiert «. Zum Schluss wird dem Coming-out, das im ganzen Artikel von "Gewicht « ist, "Gewicht « genommen. Auch eine »Freundin können die Eltern nicht mögen«. Das, worauf es letztendlich ankomme - und das ist unabhängig von Geschlecht und Sexualität - , ist, ob die Eltern den Freund mögen oder nicht. Der sexuellen Orientierung, die überhaupt erst der Grund für eine um das Coming-out angeordnete Wegbegleitung ist, wird die Relevanz genommen. Diese Aussage steht im Widerspruch zu all den Szenarien, die im Artikel diskutiert werden. Der Artikel schließt mit einem: »Ein Versuch ist es wert. >Viel Glück!««. Es ist eine Aufforderung, den Weg, auf dem der Text begleitet, einzuschlagen. Unausgesprochen aber selbstverständlich ist dabei der dritte Weg, der einzige Weg zum Glück, gemeint.

\section{Eine Wegbegleitung}

Generell wird im »Milchbüechli« stolz und selbstbewusst über die eigenen Lebens- und Seinsweisen berichtet. Damit werden den Jugendlichen positive Identifikationsmög- 
lichkeiten geboten. Aber auch die "falschsexuelle« Gemeinschaft selbst wird als stolz und selbstbewusst hergestellt (»[w]ir habens uns nicht ausgesucht, wir hatten einfach Glück«, »die beste Phase meines Lebens«). Die »falschsexuelle« Gemeinschaft ist aber keine Gemeinschaft, in der alle (schon immer) stolz und selbstbewusst sein müssen, sondern es ist ein Ort, an dem Jugendliche auf dem Weg zu einem selbstbewussten und stolzen Leben unterstützt werden. Wie sieht aber diese Unterstützung aus und was wird dabei festgeschrieben? Die Weg-Metapher ist zentral für die Art und Weise, wie im analysierten Diskursfragment über das Coming-out gesprochen wird. Zunächst einmal bezieht sich die Wegbegleitung auf das Coming-out als Moment, der auf dem Weg zur glücklichen Beziehung von Gewicht ist. Die Eltern als Adressat*innen stellen eine Hürde auf diesem Weg dar. Die Notwendigkeit eines Coming-out wird hier über das Ideal einer ehrlichen Familienbeziehung (die Eltern nicht umgehen wollen), aber auch einer glücklichen Paarbeziehung (den Freund mit nach Hause nehmen wollen) festgeschrieben. Auch nach dem Coming-out und vor allem dann, wenn sich das, worüber mensch spricht, in einer konkreten Beziehung manifestiert, ist Rücksichtnahme angesagt. Auch hier erkennen die sich im Sprechakt des Coming-out in Richtung Identität Bewegenden ihre Schuld an. Als diejenigen, als die sie sich outen, verlassen sie den vorgesehenen heteronormativen Pfad und schulden es den Eltern umso mehr, "glücklich zu sein«. Gerade nach dem Coming-out gilt es, kein Scheitern zu präsentieren. Die Anerkennung der Schuld ist der zu zahlende Preis für den Weg zum Glück, den das Coming-out verspricht. Das Coming-out und die gleichzeitige Rücksichtnahme gegenüber den Eltern werden als der Weg zur Normalität festgeschrieben. Auf dem Weg zum persönlichen Glück müssen die Jugendlichen ihre Komfortzone verlassen, um zu stolzen, handlungsmächtigen Subjekten zu werden.

\section{Happy End und die Anerkennung der Schuld}

Sowohl im »Milchbüechli« als auch in der »Out!« spielt das zu Hause Wohnen eine wichtige Rolle in Bezug auf das Coming-out. $\mathrm{Zu}$ Hause $\mathrm{zu}$ wohnen, verweist auf eine zumindest räumlich enge Beziehung zu den Eltern. Diese Nähe ist auch ein Grund, warum in beiden Erzählungen die Familie die Institution ist, in deren Richtung sich das Coming-out wendet. In beiden Fällen wird die im Coming-out vollzogene Bewegung Richtung Identität als etwas beschrieben, bei der es »etwas zu gewinnen gibt«. Beide Erzählungen sind Happy-End-Erzählungen. Zu gewinnen gibt es Normalität (eine normale Familienbeziehung). Die Notwendigkeit des Coming-out begründet sich bei der Milchjugend zum einen darin, den nächsten wichtigen Schritt in der Beziehung - »den Freund mit nach Hause nehmen « - einschlagen zu wollen, zum anderen aber auch im Anspruch an eine ehrliche (authentische) Beziehung zu den Eltern. Auch in der »Out!« wird die Notwendigkeit des Coming-out mit der ehrlichen (authentischen) Beziehung zu den Eltern begründet. Der einzige Mangel des glücklichen Familienfilmabends ist das Schweigen. Das Coming-out wird in beiden Texten als ein ehrlicher Sprechakt beschrieben, durch den mensch zu einem authentischen Subjekt wird und eine aufrichtige Familienbeziehung herstellt. Daran anschließend bedient sich insbesondere die »Out! « einer christlichen Terminologie (»Offenbarung«, »Bekennen«), die das Comingout als einen Geständnisakt festschreibt. Das Coming-out ist der Sprechakt, in dem das 
Subjekt wahr über sich selbst spricht. Es gesteht, was es immer schon gewesen ist. In beiden Artikeln geht die »Offenbarung« dessen, was mensch ist, mit der Anerkennung einer Schuld einher, die sich in die Familienbeziehung einschreibt. Die Anerkennung der Schuld äußert sich, indem die Verantwortung für das, was durch das Coming-out »Zerbrochen ${ }^{203}$ ist, übernommen wird. Den Eltern Zeit geben, den Freund nicht gleich beim ersten Date mit nach Hause nehmen, ihnen fünfmal bestimmte Dinge erklären sind Versuche, die eigene Schuld abzuarbeiten. Die Jugendlichen, die aus der Norm fallen, arbeiten zugleich gegen die Verletzungen, die damit einhergehen, dass sie aus der Norm fallen, und gegen die normativen Vorurteile der Institution Familie. Der Preis, der in der Anerkennung der Schuld zu zahlen ist, ist das Risiko, an dem zu zerbrechen, das zerbrochen ist. Auf der einen Seite bejahen die Coming-out-Erzählungen der Jugendlichen eine dem Coming-out vorausgehende Schuld, indem sie der Familie gegenüber die Schuld für das, was zerbrochen ist (der heteronormative Weg zum Glück, die glückliche Familie), anerkennen und tilgen. Auf der anderen Seite eröffnen die Happy-End-Erzählungen die Möglichkeit, durch das Coming-out ein stolzes und handlungsmächtiges Subjekt zu werden.

\subsubsection{Coming-out - ein Jugendthema?}

Das Coming-out ist ein Thema, das vor allem in queeren Jugendgruppen relevant ist. Wie aus den Diskursfragmenten deutlich wird, sind Jugendliche im Besonderen damit konfrontiert, mit der »Geburtsfamilie« zusammenzuleben und so auch abhängig von Menschen zu sein, die die eigenen Erfahrungen zumeist nicht teilen und zum Teil in normativen Vorstellungen verhaftet sind. Das Coming-out ist aber kein einmaliger Akt, der irgendwann abgeschlossen ist, sondern eine Entscheidung, die in vielen Kontexten immer wieder neu getroffen werden muss. ${ }^{204}$ Entsprechend ist das Coming-out auch in den Nicht-Jugendgruppen Thema. Für die Türkis Rosa Lila Villa ${ }^{205}$ spielte es zumindest in der Beratung immer schon eine Rolle. Das Coming-out ist ein Beratungsfokus der beiden »Tipps«. Im Vergleich zu heute stand das Thema Coming-out allerdings in den Anfängen stärker im Zentrum. ${ }^{206}$ Durch die bunte Hausfassade gilt auch das Betreten der Villa als ein »Coming-out through coming in«. Viele der Gruppen, die sich in der Villa treffen, tauschen sich auch über das Coming-out aus; es ist beispielsweise, neben Gewalt und Asyl, auch eines von zehn Themen, das eine eigene Rubrik auf der Homepage der Villa bildet. ${ }^{207}$ Hier betont die Villa, ein vielseitiges Sprechen (über Lust und Ängste) über das Coming-out eröffnen zu wollen, ohne dass mensch auf IdentitätsKategorien festgeschrieben wird. ${ }^{208}$

203 Ebd., S. $168 f$.

204 Vgl. V. Woltersdorff: Coming out, S. 19.

205 In der Villa ist die Zielgruppe unabhängig vom Alter.

206 Während sich die Beratung zu Beginn noch sehr stark um das Thema Coming-out angeordnet hatte, gab es mit der Zeit Verschiebungen. Aktuell ist das Coming-out ein Beratungsthema unter anderen.

207 Türkis Rosa Lila Villa: Coming-out, https://dievilla.at-coming-out-vom 24.11.2020.

208 In der Villa über das Coming-out zu sprechen, bedeutet, sich sowohl über »Unsicherheiten und Ängste« als auch über »Lustvolles und Schönes « auszutauschen. Auf der Website ist zudem ein 
Auch in einem Artikel der Villa aus den "Lambda-Nachrichten« vom Sommer 2014 wird über das Coming-out geschrieben. ${ }^{209}$ Dort wird die »Coming-out im Beruf!«Gruppe des »Lila Tipp« vorgestellt. Die Arbeitswelt ist ein Ort, an dem immer wieder eine Entscheidung für oder gegen ein Coming-out getroffen werden muss. Auch hier sind die Beziehungen weitgehend nicht selbstgewählt. Zu Beginn des Artikels wird die Notwendigkeit einer »Coming-out im Beruf!«-Gruppe gerechtfertigt, indem auf Studien verwiesen wird, nach denen nur »10 Prozent aller Lesben und Schwulen völlig offen an ihrem Arbeitsplatz« sind. Im Gegensatz zu den beiden Jugendgruppen, die die Notwendigkeit des Coming-out mit der Qualität der Paarbeziehung und der Ehrlichkeit begründen, ist dieser Argumentation zufolge das Coming-out zunächst einmal Selbstzweck. Es ist notwendig, weil im Umkehrschluss 90 Prozent aller Lesben und Schwulen eben nicht "völlig offen an ihrem Arbeitsplatz« sind. Aber auch im Villa-Artikel wird die Notwendigkeit des Coming-out in der ehrlichen Beziehung zu den Kolleg*innen begründet: Das Coming-out ist notwendig, um »nicht schweigen zu müssen«, wenn andere vom Wochenende erzählen. Das Coming-out wird also auch hier als Weg zu einer ehrlichen Beziehung beschrieben. Ähnlich wie in der »Out!« wird auch in diesem Artikel das Coming-out als riskanter Sprechakt festgeschrieben: Es geht damit das Risiko einher, »im Beruf benachteiligt zu werden« und von den »Kollegen nur als die Lesbe« gesehen zu werden. Das Coming-out am Arbeitsplatz wird mit dem Versprechen verknüpft, sich nicht mehr über ein Schweigen konstituieren zu müssen, sondern ehrlich und offen sein zu können. Es wird aber auch mit Ängsten verknüpft, etwas dabei $\mathrm{zu}$ verlieren - Angst vor Sanktionen, die damit einhergehen, den »heteronormativen Pfad« zu verlassen (voll und ganz auf eine Identitäts-Kategorie reduziert zu werden, Benachteiligung im Beruf). Während beim Coming-out im Kontext der Familie Sanktionen in Bezug auf die Reduktion auf eine Identitäts-Kategorie oder die Schuldigkeit, erst recht glücklich sein zu müssen, beschrieben werden, wird im Villa-Kontext noch eine weitere sanktionierende Dimension angesprochen: die Benachteiligung im Beruf. Der Preis, der in der »Umwendung Richtung Identität« bezahlt werden muss, kann auch monetärer Art sein. Sich in Richtung Arbeitsplatz zu wenden und auszurufen »das bin ich«, birgt das Risiko einer Benachteiligung am heteronormativ ausgerichteten Arbeitsmarkt.

Die Villa stellt sich als Raum dar, in dem ein Austausch über Hoffnungen (z.B. auf eine ehrliche Kolleg*innenbeziehung) und Ängste (z.B. Benachteiligung am Arbeitsplatz) stattfindet, aber auch als Ort, an dem gemeinsam Handlungsstrategien erarbeitet werden. Im Gegensatz zur »Out!« und zum »Milchbüechli«, die vermeintlich optimale Handlungsstrategien nahelegen, bleibt hier offen, welcher konkrete Umgang mit

Bild von einem Transparent mit der Aufschrift »FUCK YOUR LABELS« zu sehen. Mit diesem Bild, aber auch dem Schreiben (» [e]s kann spannend sein zwischen den oder jenseits von Geschlechtern zu stehen «; »Cefühle, Wünsche und Phantasien die heterosexuelle Begehrens- und Ceschlechternormen auf den Kopf stellen«) positioniert sich die Villa gegen eine Idee von Coming-out, nach der in Form von (Identitäts-/) Kategorien festgeschrieben werden muss, wer man ist.

209 Türkis Rosa Lila Villa: »Coming-out im Beruf!«, in: Lambda-Nachrichten vom Mai-Juni 2014, https: //lambdanachrichten.at-In142.pdf vom 15.09.2020, S. 14. 
dem Coming-out denkbar beziehungsweise undenkbar ist. ${ }^{210}$ Eine Strategie im Umgang mit dem Coming-out wird allerdings auch im Villa-Text hervorgehoben: In der Coming-out-Gruppe gibt es eine Auseinandersetzung mit der »rechtlichen Situation«. Während die Coming-out-Erzählungen der Jugendlichen - in Anerkennung der Schuld - Strategien für das, was durch das Coming-out in der Familie zerbrochen ist, eröffnen, wird im Villa-Text der Rechtsweg als Strategie gegen das, was durch das Comingout ungerechtfertigter Weise zerbrechen (die berufliche Perspektive) kann, nahegelegt. Nicht die eigene Schuld wird anerkannt, sondern das Recht, im Recht zu sein, wird eingefordert.

Auch bei TrIQ und LesMigraS gibt es Orte des Austauschs, der Unterstützung und der Entwicklung von gemeinsamen Handlungsstrategien. Diese sind aber weniger um den Begriff des Coming-out, sondern - vor allem im Fall von LesMigraS - um den Begriff des Empowerment sowie in Bezug auf die jeweils spezifischen Arbeitsbereiche der Projekte (Gewalt, Mehrfachdiskriminierung, Trans* und Inter*) angeordnet. So werden beispielsweise im Rahmen des Empowerment-Projekts »Tapesh« von LesMigraS Räume geschaffen, in denen der Austausch zu Gewalt und Diskriminierung und die Entwicklung gemeinsamer Handlungsstrategien möglich sind. Obwohl oder gerade weil das Coming-out bei LesMigraS nicht zentral ist, positioniert sich LesMigraS am Internationalen Coming-out-Tag in einer Pressemitteilung zu diesem Thema. ${ }^{211}$ Anders als in den anderen vier Projekten ist das Sprechen über das Coming-out von LesMigraS eine Positionierung gegen ein Coming-out-Verständnis der Community. Das Coming-out scheint für LesMigraS nicht so irrelevant zu sein, dass dieses Thema ignoriert werden kann. Auch in einer Positionierung gegen das Coming-out wird eine Beziehung zwischen LesMigraS und dem Coming-out hergestellt. In ihr schreibt sich fort, was sich bereits in der Positionierung gegen den CSD oder Rassismus in der Community gezeigt hat. LesMigraS konstituiert sich auch in Bezug auf den Coming-out-Begriff als ein Wir, das beständig die Ausschlüsse der Community benennt. Zunächst einmal zeigt LesMigraS, auf welcher Ebene eine bejahende Beziehung zum Coming-out möglich ist. Gleich zu Beginn der Pressemitteilung bejaht die Einrichtung die Notwendigkeit eines Coming-out auf individueller Ebene:

»Auch 2013 sind lesbische, schwule, bisexuelle und trans* (LSBT*) Lebensweisen in Deutschland keine Selbstverständlichkeit. Nach wie vor sind Heterosexualität und CisGeschlechtlichkeit (eine Identifikation mit dem Ceschlecht, das bei Geburt zugewiesen wurde) die Norm, die Abweichung muss meistens immer noch explizit benannt werden. Coming-Out ist deswegen für viele LSBT* eine Notwendigkeit, um ihre Lebensweise in ihrem sozialen Umfeld - in der Herkunftsfamilie, im Freund_innenkreis, auf Arbeit, im Sportverein - sichtbar zu machen.« konkrete Austausch-Gruppe zu informieren, während die Artikel aus »Milchbüechli« und »Out!« bereits selbst konkrete Orte des Erfahrungsaustauschs sind.

211 LesMigraS: Internationaler Coming-Out-Tag am 11.10.2013. Pressemitteilung der Lesbenberatung e.V. Berlin 2013, https://lesmigras.de-tl_files-lesmigras-pressemitteilungen/PM_2013_Coming_Out. pdf vom 24.11.2020. 
LesMigraS zufolge ist ein Coming-out auf einer individuellen Ebene notwendig, um aufgrund bestehender Heteronormativität - im sozialen Umfeld sichtbar zu sein. Dabei wird das Coming-out als »Prozess« verstanden. Das bedeutet, die Entscheidung für oder gegen ein Coming-out muss immer wieder neu getroffen werden.

Anders als in den Jugenddiskursen und der Villa begründet LesMigraS die Notwendigkeit eines individuellen Coming-out nicht mit der Notwendigkeit einer ehrlichen Beziehung, sondern mit der Norm, in der das Coming-out die Voraussetzung von Sichtbarkeit ist. Im Gegensatz zum »Out!«-Artikel, in dem das Coming-out durch eine Unterscheidung zwischen Coming-out und Post-Coming-out als einmaliger Sprechakt festgeschrieben wird, versteht LesMigraS das Coming-out als einen dauerhaften Prozess. Ähnlich, wie es in den anderen drei Einrichtungen der Fall ist, sagt auch LesMigraS Ja zu einem individuellen Coming-out. Die Einrichtung wendet sich allerdings gegen ein individuelles Coming-out, das als politische Strategie ein Versprechen auf ein "glückliches«, »selbstbestimmtes« Leben und Toleranz durch "persönlichen« Kontakt mit sich bringt. LesMigraS sagt Nein zum Coming-out als einziger Strategie, »Ausgrenzung zu begegnen «, und Nein zu einem Wissens-Regime, demzufolge der einzig wahre/richtige Weg zu einem "glücklichen und selbstbestimmten Leben« das Coming-out ist.

LesMigraS »verkompliziert« das in der Kritik stehende Coming-out-Verständnis, indem die Einrichtung - basierend auf eigenen Studienergebnissen - das Comingout-Verständnis um Begriffe wie »Ängste«, »Diskriminierung«, »Mehrfachzugehörigkeiten«, »Druck«, »Passing«, »rechtliche« Notwendigkeiten und »zugeschriebene Sichtbarkeiten« erweitert. Die Einrichtung fügt dem gängigen Coming-out-Verständnis auch die Community als möglichen Adressat*innenkreis hinzu: "Auch in LSBT*Communities müssen sich deswegen manche >outen<, wenn sie nicht von allen als LSBT* $^{*}$ wahrgenommen werden, wie z.B. Femmes, ältere LSBT*, Trans*-Personen mit (erfolgreichem) Passing«. LesMigraS konstituiert sich als ein Wir, das im Gegensatz zum gängigen Coming-out-Verständnis eine vielschichtigere, komplexere Vorstellung vom Coming-out hat.

Bei TransInterQueer e.V. spielt das Coming-out zumindest in den produzierten Broschüren, politischen Stellungnahmen, Flyern und Artikeln kaum eine Rolle. Damit ist nicht gesagt, dass das Coming-out für TrIQ nicht relevant ist, der inhaltliche Fokus des Textmaterials ist aber ein anderer. Adressat*innen der Aufklärungs- und Sensibilitätsbroschüren sind primär Gesellschaft, Mediziner*innen, Medien, Psychotherapeut*innen u.v.a.m. Die politischen Stellungnahmen sind hingegen vor allem Positionierungen gegen rechtliche, medizinische und mediale Diskurse. TrIQ produziert aber kaum oder wenig Textmaterial, in dem die Art des Erfahrungsaustauschs in Bezug auf das individuelle Befinden der eigenen Mitglieder verhandelt wird. Die Hinweise in der Broschüre "Intersektionale Beratung von/zu Trans* und Inter* " ${ }^{212}$ verweisen darauf, wie TrIQ selbst als Beratungsort angeordnet ist. Hier steht nicht das Coming-out, sondern Beratung zu medizinischen und rechtlichen Themen im Vordergrund. Ähnlich wie bei LesMigraS wird auch in dieser Broschüre Empowerment als handlungsleitend ausgewiesen: 
»Empowerment ist in unserem Verständnis ein zentraler Ansatz in der Beratungsarbeit. Dabei steht die Selbstermächtigung und Selbstbestimmung der Person mit Diskriminierungserfahrungen im Vordergrund. Das kann in der Beratungspraxis bedeuten, dass gemeinsam mit der Person Ressourcen besprochen und Handlungsstrategien entwickelt werden, um einen individuellen und bestärkenden Umgang mit diskriminierenden Erfahrungen zu finden. Dabei ist es immer wieder wichtig, die Bedürfnisse der Person zu erfragen und diese als Ausgangspunkt zu begreifen. Empowerment bedeutet auch, Personen den Raum zu geben, sich zu vernetzen und gegenseitig zu unterstützen. ${ }^{213}$

Nicht die Unterstützung zum Coming-out als individuelle Ermächtigung, sondern die Ermächtigung zur individuellen Selbstermächtigung in Bezug auf Diskriminierungserfahrungen ordnen diese Austauchorte an.

Der einzige Ort, an dem das Coming-out bei TrIQ erwähnt wird, ist die Website. Hier wird in den Ausführungen darüber, warum das Projekt TrIQ gebraucht wird, das Coming-out als wichtiger Aspekt der psychosozialen Situation von Trans*- und Inter*Personen genannt. ${ }^{214}$ Anders als im Ratgeber zur Trans* - und Inter*-Beratung scheint für die Einrichtung selbst das Coming-out auf einer individuellen Ebene eine Rolle zu spielen. TrIQ thematisiert in diesem Zusammenhang zunächst das sogenannte »innere« Coming-out, als einen Prozess, der mit »Ängsten und »Orientierungslosigkeit« verknüpft ist. Nicht auf den vergeschlechtlichten Platz zu passen, der einem zugewiesen wird, macht TrIQ als Ort der psychosozialen Unterstützung notwendig. Im Umkehrschluss kann die psychosoziale Unterstützung dazu beitragen, Ängste abzubauen und die »Orientierung« wiederzufinden. TrIQ positioniert sich zudem als einen notwendigen Unterstützungsort nach dem Coming-out: »Nach einem Trans-Coming-out fangen die Probleme allerdings oft erst richtig an, auch für die Angehörigen. Da die Reaktionen Anderer aufgrund der Tabuisierung dieser Thematik oft ablehnend ausfallen, zerbrechen am Coming-out noch immer die meisten Beziehungen, Familien und Arbeitsverhältnisse.«Auch hier wird das Coming-out als abgeschlossener Prozess hergestellt, gibt es doch ein Post Coming-out. Das Coming-out hat, indem es auf eine bestimmte Art und Weise dargestellt wird, die primäre Funktion, die Arbeit von TrIQ $\mathrm{zu}$ legitimieren.

TrIQ antwortet auf die Notwendigkeiten, die mit den Problemen um das Comingout einhergehen. Dabei grenzt TrIQ das Trans*-Coming-out vom homosexuellen Coming-out $a b$. Im Gegensatz zum homosexuellen Coming-out würden mit dem Trans*-Coming-out die Probleme erst anfangen. TrIQ spricht im Unterschied zum »Milchbüechli« oder zur »Out!« nicht vom Coming-out als Erfolgsgeschichte, sondern als Bruch-Geschichte: »Familien«, »Arbeitsverhältnisse«, und »Beziehungen« »zerbrechen am Coming-out noch immer«. Auch TrIQ bejaht das Coming-out auf einer individuellen Ebene. Es ist ein wichtiger Aspekt der psychosozialen Situation und macht die Arbeit von TrIQ notwendig. Im Gegensatz zur Villa, die sowohl das Lustvolle, Hoffnungsvolle als auch das Negative des Coming-out betont, stehen im Fall von TrIQ

214 TransInterQueer e.V.: Warum dieses Projekt gebraucht wird. 
die Ängste und Sorgen im Vordergrund. Für TrIQ ist das Coming-out eine BruchGeschichte, die im Gegensatz zu den hier analysierten Berichten der Jugendlichen kein Happy End verspricht.

\subsubsection{Das Coming-out in seiner psychosozialen Dimension}

Die beiden Coming-out-Artikel aus den Jugendzeitschriften verstehen sich als Unterstützung für andere Jugendliche. Während der »Milchbüechli«-Artikel sich explizit als Wegbegleitung darstellt, benennt der »Out!«-Artikel das Ziel, die Leser*innen auf die Überraschungen vorzubereiten, die die Autor*in selbst erlebt hat. Beide Artikel ermutigen zum Coming-out, indem sie durch eine Happy-End-Erzählung die Möglichkeit aufmachen, durch das Coming-out zu einem glücklichen, handlungsmächtigen Subjekt $\mathrm{zu}$ werden. Den Jugendlichen wird die Möglichkeit eröffnet, sich insofern als handlungsmächtig zu konstituieren, als sie den Mut aufbringen, auszusprechen, »wer sie sind «, und es ihnen gleichzeitig gelingt, Normalität in der Familienbeziehung (wieder-)herzustellen. ${ }^{215}$ Sie können zu glücklichen Subjekten werden, weil sie schlussendlich den Freund mit nach Hause nehmen können und beim Filmschauen aussprechen können, wie heiß das »Bond-Girl« sei. Der Endpunkt, der in der Happy-End-Erzählung festgeschrieben wird - die normale Familienbeziehung -, sowie die Notwendigkeiten, die das Coming-out begründen - eine ehrliche Familienbeziehung und eine glückliche Paarbeziehung -, bleiben unhinterfragt. Stattdessen wird im Namen dieser Notwendigkeiten eine eindeutige Bewegung Richtung Identität bejaht. Anstatt sich zu befreien, binden die Jugendlichen sich an die - der Bewegung Richtung Identität - vorausgehende Schuld. Es werden auch nicht die Bedingungen des Coming-out - Heterosexualität als Norm - hinterfragt, sondern die Jugendlichen bekennen sich vor dem Gesetz (der »Norm«) als schuldig. Diese Schuld schreibt sich in die Art und Weise, wie die Familienbeziehung im Kontext des Coming-out festgeschrieben wird, ein. Die Jugendlichen schulden es ihren Eltern, ehrlich zu sein. Damit ist das Coming-out auf der einen Seite eine Entscheidung, sich nicht durch ein Schweigen konstituieren zu lassen. Auf der anderen Seite bedeutet »das Schweigen brechen«, die eigene sexuelle Orientierung zu »offenbaren«. Eine ehrliche Familienbeziehung erfordert, dass die Jugendlichen über sich selbst wahrsprechen. Im Namen einer ehrlichen Familienbeziehung wird den Jugendlichen das Coming-out als eine Geständnispraxis nahegelegt. Der wahre Diskurs im Bereich der Sexualität ist - wie Foucault aufgezeigt hat - ein Diskurs des Subjekts über sich selbst. Dieser ist mit dem Christentum zu einer Geständnispraxis geworden, der eine Schuld vorausgeht. ${ }^{216}$

215 Im Artikel »Das Coming-out zwischen (Selbst-) Ermächtigung und »Geständnispraxis« wird aus einer erziehungswissenschaftlichen Perspektive ausführlich diskutiert wie sich die aktivistischen Jugendgruppen in einem solchen Sprechen über das Coming-out nicht nur auf bestimmte Art und Weise als alternativer Bildungsort konstituieren, sondern auch das pädagogische Familienverhältnis neu einschreiben. Tanja Vogler: „Das Coming-out zwischen (Selbst-)Ermächtigung und `Ceständnispraxis<-Eine diskursanalytische Betrachtung aktivistischer Coming-out-Diskurse am Beispiel von zwei queeren Jugendzeitschriften«, Jahrbuch erziehungswissenschaftliche Geschlechterforschung, 17 (2021), S. 171-183.

M. Foucault: Subjektivität und Wahrheit, S. $29 f$. 
Die Schuldigkeit vor dem Gesetz wird in den Coming-out-Diskursen der Jugendlichen insofern anerkannt, als die Diskurse den Jugendlichen nahelegen, für das, was durch das Coming-out zerbricht, verantwortlich zu sein. Die Schuld für das, was durch das Coming-out zerbrochen ist - die normale Familienbeziehung -, wird auf Seiten derer verortet, die dieses Zerbrechen vermeintlich durch ihren Sprechakt ausgelöst haben. Die Jugendlichen tilgen ihre Schuld, indem sie die Verletzungen auf sich nehmen und gleichzeitig reparieren, was zerbrochen ist. Sie übernehmen die Verantwortung für das, was zerbricht (normale Familie, der heteronormative Pfad zum Glück), und konstituieren sich so in Anerkennung der Schuld als handlungsmächtig. Der Preis dafür ist eine nervenaufreibende emotionale Arbeit, die sich unter anderem darin äußert, dass die Jugendlichen den Eltern immer wieder erklären müssen. Nicht alle Jugendlichen sind in der Lage, diesen emotionalen Preis zu zahlen und so durch das Coming-out zu einem handlungsmächtigen Subjekt zu werden. Oft sind es vor allem mehrfachmarginalisierte Jugendliche, die in anderen Bereichen bereits mit Marginalisierungserfahrungen konfrontiert sind, für die diese zusätzliche nervenaufreibende Arbeit überfordernd ist. Es ist eine Frage der sozialen, emotionalen und ökonomischen Ressourcen, ob der Weg zum Glück, der hier eröffnet wird, beschritten werden kann. Es ist aber auch die Frage, ob das Coming-out ein Happy End verspricht. Einen bestimmten Weg zum Glück vorzugeben, kann Sara Ahmed zufolge auch Druck ausüben: »Happiness is a way of being directed toward those things that would or should make you happy. Happiness can thus also be a form of pressure. Pressure does not always feel harsh. A pressure can begin with a light touch. A gentle encouragement: Go this way, go that way. Be happy, don't be happy. «"17

Die beiden Jugendprojekte konstituieren sich als Orte, an denen der Erfahrungsaustausch und die Unterstützung der Einzelnen die Möglichkeit eröffnen, zu einem "glücklicheren«, »stolzeren« und handlungsmächtigeren Subjekt zu werden. Allerdings gibt die Wegbegleitung durch das Coming-out den Weg zum Erfolg vor und schließt damit jene Menschen aus, für die dieser Weg keine Option ist. Zudem stellt sich die Frage, inwieweit, bei einer Bewegung Richtung Identität durch Anerkennung der eigenen Schuld, von Befreiung gesprochen werden kann. Allerdings steht nicht das Comingout als politische Strategie - bei der es um Befreiung gehen sollte - im Vordergrund der jugendlichen Coming-out-Diskurse, sondern das Coming-out in seiner individuellen, psychosozialen Dimension.

Im Gegensatz zur »Out!«, in der das Coming-out als Sprechakt, in dem ausgesprochen wird, was mensch immer schon gewesen ist, festgeschrieben wird, verknüpft die Villa ein Sprechen über das Coming-out mit einer »Fuck Labels«-Haltung. Das Comingout soll so jenseits einer eindeutigen Bewegung Richtung Identität denkbar sein. ${ }^{218}$ Die Villa-Diskurse halten aber nicht nur offen, was durch das Coming-out ausgesprochen wird, sondern betonen auch einen möglichst offenen Austausch über das Coming-out. Es wird explizit hervorgehoben, dass über Schönes und »Lustvolles« genauso

217 S. Ahmed: Living a Feminist Life, S. 49.

218 Wird ein solcher Versuch der Öffnung zur dogmatischen Haltung, läuft dies Gefahr, all jene auszuschließen, die sich den auf Schließung abzielenden Identitäts-Kategorien unterwerfen wollen. 
wie über Angstmachendes gesprochen werden kann. Das Sprechen über das Comingout ist anders als in den Jugend-Diskursen keine reine Happy-End-Geschichte. Es wird aber ebenfalls vom Coming-out, als einem »ehrlichen« Sprechakt, der das Schweigen bricht (endlich vom Wochenende erzählen), gesprochen. Auch hier ist die ehrliche Beziehung zu den anderen - in dem Fall den Arbeitskolleg*innen - die Notwendigkeit, auf die das Coming-out antwortet. Auch hier bedeutet das Coming-out auf der eine Seite die Möglichkeit, sich nicht mehr über ein Schweigen konstituieren zu müssen, auf der anderen Seite wird auch hier ein nicht-geoutetes Subjekt in Abgrenzung zum ehrlich sprechenden geouteten Subjekt für immer auf den Platz des Nichtauthentischen verwiesen. Nur das Subjekt, das dem sozialen Umfeld »offenbart«, wer es wirklich ist, ist authentisch. Auch hier scheint das psychosoziale Wohlbefinden, das mit der Möglichkeit, sich nicht am Arbeitsplatz verstecken zu müssen, einhergeht, im Vordergrund zu stehen. Im Gegensatz zu den Jugend-Diskursen wird dabei aber keine dem Comingout vorausgehende Schuld bejaht. Im Gegenteil eröffnet die gemeinsame Auseinandersetzung mit der rechtlichen Situation die Möglichkeit, das Recht einzufordern, nicht den Arbeitsplatz zu verlieren - eben, weil mensch unschuldig ist.

Anders als die anderen Einrichtungen sagt LesMigraS Nein zu einem Coming-out als einzige Strategie. Dabei entkoppelt LesMigraS den Coming-out-Begriff von den ausschließenden und problematischen Bedeutungen gängiger Coming-out-Diskurse. LesMigraS hinterfragt Bedeutungen, die mit dem Coming-out verknüpft sind und auf Schließung abzielende Elemente beinhalten, wie zum Beispiel die Vorstellungen vom Coming-out als einzigen Weg zum »Glück« oder zur »Selbstbestimmung« oder auch als politische Strategie. In Abgrenzung dazu verknüpft LesMigraS das Coming-out mit anderen, komplexeren Erzählungen. Indem aufgezeigt wird, wer in und durch einen verengten Coming-out-Diskurs nicht repräsentiert ist, öffnet die Einrichtung das Sprechen über das Coming-out für diejenigen, die darin nicht vorkommen. Die Einrichtung fordert eine Öffnung für diejenigen, für die das Coming-out nicht zentral ist, indem ein bestimmtes Coming-out-Verständnis abgelehnt wird. Dabei wird der Coming-out-Diskurs auf das, was er üblicherweise ist, im Sprechen von LesMigraS selbst verengt. LesMigraS wendet sich gegen ein Coming-out-Verständnis, wie es der Einrichtung zufolge »häufig« vorkommt. Zumindest in den bisher betrachteten Coming-out-Diskursen war zwar ein Verständnis vom Coming-out als befreiend und als Weg zu einem glücklichen Leben präsent, nicht aber ein Verständnis vom Coming-out als politische Strategie. Alle Einrichtungen sagen, wie auch LesMigraS, nur Ja zu einem individuell notwendigen Coming-out. LesMigraS konstituiert sich, in Abgrenzung zu denen, für die das Coming-out als individualisierende politische Strategie der einzige Weg zum glücklichen Leben ist, als ein Wir, das insofern offener ist, als die Einrichtung ein komplexeres Coming-out-Verständnis und vielschichtige Strategien verfolgt. Die Grenze, die hier gezogen wird, scheint allerdings nicht der Komplexität und Vielschichtigkeit der Coming-out-Diskurse in der Community Rechnung zu tragen. Vielmehr wendet sich LesMigraS gegen einen Coming-out-Begriff der »Gay Liberation«, der zumindest in aktuellen queeren Diskursen so kaum mehr vorkommt.

Auch LesMigraS sagt explizit Ja zu einem Coming-out, das aufgrund der Wirkmächtigkeit gesellschaftlicher Normen auf individueller Ebene notwendig ist. Anders als in 
den anderen Projekten wird die Notwendigkeit jedoch nicht in einer ehrlichen Beziehung, sondern in den gesellschaftlichen Normen, die Sichtbarkeiten produzieren, festgeschrieben. Damit werden die das Coming-out überhaupt erst notwendig machenden Normen in den Blick genommen. So muss das Coming-out nicht an das Versprechen, ein ehrliches Subjekt zu werden, gebunden werden - ein Versprechen, das Menschen, die sich nicht outen wollen, die Möglichkeit verwehrt, ein ehrliches Subjekt zu werden. Genauso wenig wird eine Schuld vor der Norm anerkannt.

TransInterQueer erwähnt das Coming-out, um sich selbst als notwendigen Ort der psychosozialen Unterstützung zu legitimieren. Im Gegensatz zum »Milchbüechli« und zur »Out!« wird nicht das Happy End, sondern die Bruch-Geschichte betont. Die fragile Coming-out-Darstellung ist die umgekehrte Seite der Medaille der Erfolgsgeschichte. Sie macht wenig Hoffnung und spricht nicht über diejenigen, denen das Coming-out Erfolg verspricht oder die sich durch das, was sie durch das Coming-out geworden sind, als erfolgreiche Subjekte konstituieren. Zerbrechliche Geschichten laufen zudem Gefahr, Politiken des Mitleids ${ }^{219}$ auf den Plan zu rufen. Gleichzeitig sprechen sie in einer Welt, in der mensch glücklich zu sein hat, über Unglückliches und Zerbrochenes und brechen so mit dem, was sagbar ist. ${ }^{220}$ Auch auf einer affektpolitischen Ebene stellt eine Bruch-Geschichte einer "gay as in happy«-Politik Politiken negativer Affekte entgegen, die in einer weißen westlichen Welt nicht vorgesehen sind. ${ }^{221}$ Die Bruch-Geschichte wird zudem implizit - in Abgrenzung zur Homosexualität - als trans* spezifisch ausgewiesen. Damit wird auf der einen Seite das Narrativ des erfolgreichen homosexuellen Coming-out und auf der anderen Seite das Narrativ des nicht-erfolgreichen Trans* Coming-out festgeschrieben. Diese Eindeutigkeiten ignorieren, dass sowohl homosexuelle als auch Trans*-Coming-outs erfolgreich sowie nicht erfolgreich sein können. Es werden unnötige Differenzen produziert, um ein trans"spezifisches Projekt zu legitimieren, das auch auf anderer Ebene legitimiert werden könnte.

Alle fünf Gruppen konstituieren sich als politische Subjekte, indem sie davon sprechen, auf eine bestimmte Art und Weise in der Öffentlichkeit - auf der Straße - zu erscheinen oder nicht zu erscheinen (Pride). Sie konstituieren sich auch als ein Wir, das in Bezug auf sich selbst und das gemeinsame Handeln solidarisch ist (Rassismus). Die Projekte konstituieren sich aber auch als eine Gemeinschaft, indem sie als Austausch- und Unterstützungsorte bestimmte Erfahrungsräume und individuelle Handlungsstrategien eröffnen. Das Coming-out ist ein Thema, um das sich die Projekte als Austauschund Unterstützungsorte anordnen. Dabei ist das Spezifische am Coming-out-Diskurs - verglichen mit den Analysen zur Pride und zur Mehrfachdiskriminierung -, dass er sich weniger um die Frage nach dem Politischen, sondern mehr um die Frage nach dem individuellen, inner-psychischen Wohlbefinden anordnet. Denn anders als zu Zeiten der bewegungsgeschichtlichen Anfänge des Begriffs wird das Coming-out nicht als

219 W. Brown: Wounded Attachments.

220 S. Ahmed: Living a Feminist Life, S. 57.

221 Vgl. A. Cvetkovich: Depression ist etwas Alltägliches: Öffentliche Gefühle und Saidiya Hartmans >Lose Your Mother<. 
politische Strategie, sondern als individuelle Notwendigkeit bejaht. Die jeweiligen Gemeinschaften konstituieren sich im Sprechen über das Coming-out als Wir, indem bestimmte Möglichkeiten, »zum Subjekt zu werden«, eröffnet und andere verschlossen werden.

Vor dem Hintergrund, dass in diesen Diskursen das individuelle Wohlbefinden im Vordergrund steht, ist auch die Debatte um die Frage nach der Rolle der Identität ein andere. Im Namen eines individuellen Wohlbefindens sagen alle Projekte Ja zu einem Coming-out als Bewegung Richtung Identität und damit zu dem "Lauf des Sexualitätsdispositivs «. ${ }^{222}$ In den Jugendprojekten wird dabei sogar die dem Coming-out vorausgehende Schuldigkeit anerkannt, während die Villa dazu ermächtigt, die eigene Unschuld einzufordern. Die Diskurse der Projekte unterscheiden sich darin, inwiefern das Coming-out als Möglichkeit eröffnet wird, zu einem handlungsmächtigen, erfolgreichen Subjekt zu werden. In den Jugendgruppen ist die Erfolgsgeschichte eindeutig mit diesen Möglichkeiten, zum Subjekt zu werden, verknüpft. Die Bruch-Geschichte von TrIQ verweist hingegen eher darauf, durch das Coming-out zu einem Subjekt zu werden, das der Unterstützung durch die Einrichtung bedarf. ${ }^{223}$ Die Villa spricht auf der einen Seite von der Möglichkeit, durch das Coming-out zu einem ehrlichen Subjekt $\mathrm{zu}$ werden, auf der anderen Seite von der Möglichkeit, durch das Einfordern der eigenen Unschuld zu einem handlungsmächtigen Subjekt zu werden. LesMigraS hingegen lehnt das Coming-out als einzigen Weg zum Glück ab und spricht von der Möglichkeit des psychosozialen Wohlbefindens durch Sichtbarkeit. Die Einrichtung lehnt aber auch eine dem Coming-out vorausgehende Schuld ab, wenn die Norm selbst als Grund für das Coming-out in den Blick genommen wird.

\subsection{Das ambivalente queer-politische Subjekt}

Die Art und Weise, wie die queeren Einrichtungen sich in den Verhandlungen der drei Themen - Pride-Paraden, Mehrfachdiskriminierung und Coming-out - als ein kollektives Wir konstituieren, ist mitunter sehr unterschiedlich. Es zeigt sich allerdings, dass es in den queeren Diskursen bestimmte Verschiebungen gegeben hat. Es werden beispielsweise Mehrfachidentitäten, Affekte und Prekaritäten statt einfache Identitäten ins Zentrum der gemeinsamen Politiken gesetzt. Ferner sind postkoloniale, intersektionale und Critical-Whiteness-Debatten ein wesentlicher Bestandteil queerer Diskurse, und das Coming-out wird zur individuellen statt politischen Strategie. Gleichzeitig verweisen die drei am meisten verhandelten Themen - insbesondere das Coming-out und die Pride-Paraden - aber auch auf bewegungsgeschichtliche Kontinuitäten. Was die

222 M. Foucault: Der Wille zum Wissen, S. 151.

223 Insbesondere bei TrlQ und LesMigraS scheint der Empowerment-Begriff den Coming-out-Begriff ersetzt zu haben. Während das Coming-out durch ein Wahrsprechen über sich selbst Handlungsmächtigkeit verspricht, bezeichnet Empowerment den gemeinsamen Versuch, Handlungsstrategien im Umgang mit Diskriminierung zu suchen, durch die die einzelne Person handlungsmächtig wird. Individuelles Wohlbefinden und Handlungsmächtigkeit werden nicht erreicht, indem die Menschen aussprechen, wer sie sind, sondern indem sie Strategien im Umgang mit der Diskriminierung, die sie erfahren, finden. 
Frage nach der Rolle von Identitäten in den untersuchten queeren Gemeinschaftskonstruktionen betrifft, so zeigen die Verhandlungen, dass keine einfache Antwort möglich ist. Identitäten spielen eine Rolle, sie spielen mitunter aber auch keine Rolle. Und in den vielfältigen Versuchen, auf die Ein- und Ausschlüsse eindeutiger Identitätspolitiken zu antworten, werden erneut Schließungen produziert.

Den Auswahlkriterien entsprechend agieren alle fünf Projekte sowohl auf einer nach innen gerichteten unterstützenden Ebene als auch auf einer über die eigene Gemeinschaft hinausgehenden Ebene des Politischen. Die Grenzziehungen zwischen den Ebenen verlaufen nicht glatt; vielmehr beeinflussen und konstituieren sie sich wechselseitig. Es ist auch erkennbar, dass - je nach Thema, das verhandelt wird - eine der beiden Ebenen dominanter sein kann. Dabei zeigt sich auf allen Ebenen, dass die queeren Projekte die vielschichtigen Kritiken an schwul-lesbischen, queeren und feministischen Identitätspolitiken wahrgenommen haben und versuchen, auf diese $\mathrm{zu}$ antworten. ${ }^{224}$ Mit den Antworten entkommen sie vielen der kritisierten identitätspolitischen Schließungen, sie geraten aber auch in neue Widersprüche. Es zeigt sich auch, dass sich geographische Orte, der Grad der Institutionalisierung, die Entstehungsgeschichte, aber auch die Arbeitsschwerpunkte - Aspekte, in denen sich die Projekte unterscheiden in die Aushandlungen eines kollektiven Wir einschreiben. So spielen beispielsweise bei den Berliner Projekten die Debatten, die in der Berliner Community geführt werden, und Forderungen, die speziell an diese Community gerichtet werden, eine wichtige Rolle. In der Milchjugend scheint - stärker als bei den anderen Einrichtungen - im Erbe der "Gay Liberation« eine queere Politik des Stolzes zentral zu sein. Die Spezifika der VillaPolitiken sind nicht nur eng verknüpft mit der Geschichte der Villa, sondern auch mit den Möglichkeitsbedingungen, die die Stadt Wien zur Verfügung stellt. ${ }^{225}$ Insgesamt haben sich drei Themen herauskristallisiert, die in allen Projekten verhandelt werden: Pride-Paraden, Mehrfachdiskriminierung und das Coming-out.

Gemeinsam ist den Diskursen zu den Pride-Paraden und zur Mehrfachdiskriminierung, dass als Antwort auf die Kritik an eindeutigen Identitätspolitiken Affekte, Körper, Prekarität und Mehrfachidentitäten eingesetzt werden. Dabei schließen die Verhandlungen der fünf queeren Projekte insbesondere - allerdings auf unterschiedliche Art und Weise - an postkoloniale, intersektionale und Critical-Whiteness-Diskurse an: Queere Projekte konstituieren sich in den Verhandlungen zu den Pride-Paraden insofern als kollektives Wir, als sie sich, in Abgrenzung zu einem erinnerungspolitisch festgeschriebenen politischen Subjekt der Pride, selbst als offen herstellen, indem das politische Subjekt »Pride« zum Ort der Forderungen nach Öffnung gemacht wird. Das passiert vor allem, indem die aus den Erinnerungen und bis heute aus den Erinnerungsveranstaltungen ausgeschlossenen Mehrfachidentitäten benannt werden, um ebendiese Ausschlüsse sichtbar zu machen. Die queeren Einrichtungen verhandeln die Pride aber nicht nur als Ort des Ausschlusses, sondern auch als das, was sie in neoliberalen Zeiten geworden ist. Gemeinsam ist beiden Verhandlungen, dass im Namen dessen,

224 Auch das war zu erwarten, wurden doch entsprechend Projekte ausgewählt, die sich als »queer« im identitätskritischen Sinne verstehen.

225 Z.B. günstiger Pachtvertrag, Wohnungen für Geflüchtete, vergleichsweise wenig Restriktionen auf der Regenbogenparade. 
was die Pride immer schon gewesen ist - dessen, was sie eigentlich ist -, abgelehnt wird, was die Pride aktuell ist: Die Pride war immer schon politisch, nicht kommerziell, und sie ist eigentlich mehrdimensional, nicht eindimensional. Oft tendieren die Projekte dabei dazu, bereits allzu genau zu wissen, was der CSD eigentlich ist oder sein sollte. Auch bei den Versuchen, jenseits von Sprache, die uns repräsentierbar macht, auf Körperlichkeiten und Affekte zu rekurrieren, bleibt die Schwierigkeit, dass sich nicht alle Körper im Namen dieses queeren Wir versammeln können und nicht alle dessen affektiven Anrufungen folgen können. Körper, die nicht dazu in der Lage sind, gleichzeitig Transparente und Flyer zu halten oder an der Euphorie der Pride teilzuhaben, bleiben außen vor. Die queeren Einrichtungen konstituieren sich auch insofern als kollektives Wir, als die Thematisierung von Rassismus und Mehrfachdiskriminierung in den Vordergrund gerückt und an den Solidaritätsbegriff geknüpft wird. Sie stellen sich als solidarisches Wir her, das gleichzeitig offen und selbstkritisch ist und im Namen von Solidarität Forderungen stellt. Dabei richten sich die Forderungen im Namen von Solidarität bevorzugt an die Community. Durch diese Forderungen konstituieren sich die Einrichtungen im doppelten Sinne als solidarisch: Sie sind insofern solidarisch, als sie Solidarität fordern, sie konstituieren sich aber auch in Abgrenzung zu denen, an die die Forderungen gerichtet werden, als solidarisch. Dabei passiert es mitunter, dass die queeren Projekte sich selbst in Abgrenzung zu denen, von denen Solidarität eingefordert wird, als das "gute«, solidarische Wir herstellen. Zuletzt konstituieren die queeren Einrichtungen ein kollektives Wir, für das das Coming-out nicht mehr politische Strategie, sondern primär individuelle Notwendigkeit ist. Im Namen dieser individuellen Notwendigkeit eröffnen die Unterstützungs- und Austauschorte der Einrichtungen die Möglichkeit, durch das Coming-out zu einem handlungsmächtigen und glücklichen Subjekt zu werden. Das Coming-out ist als individueller Akt sagbar und zentral, insofern es ermöglicht, von der »Norm« abweichende Personen auf dem Weg zur psychosozialen Gesundheit zu unterstützen. Das individuelle Wohlbefinden steht über einer Kritik an eindeutigen Identitäten. Allerdings laufen auch hier bestimmte von den Einrichtungen eröffnete Möglichkeiten, durch das Coming-out zu einem handlungsmächtigen und glücklichen Subjekt zu werden, Gefahr, Schließungen zu produzieren. Beispielsweise wenn die dem Coming-out vorausgehende Schuld bejaht wird oder wenn eine einseitige Happy-End- oder Bruch-Geschichte erzählt wird. 
